\title{
Algoritmos para problemas de programação não-linear com variáveis inteiras e contínuas
}

\author{
Rafael Durbano Lobato
}

DisSERTAÇÃO APRESENTADA

$\mathrm{AO}$

Instituto De Matemática e Estatística

DA

UniversidAde DE SÃO PAUlO

PARA

OBTENÇÃO DO TÍTULO

$\mathrm{DE}$

Mestre em Ciências

Programa: Ciência da Computação

Orientador: Prof. Dr. Ernesto G. Birgin

Durante o desenvolvimento deste trabalho o autor recebeu auxílio financeiro da FAPESP

São Paulo, junho de 2009 



\section{Algoritmos para problemas de programação não-linear com variáveis inteiras e contínuas}

Este exemplar corresponde à redação final da dissertação devidamente corrigida e defendida por Rafael Durbano Lobato e aprovada pela Comissão Julgadora.

Banca Examinadora:

- Prof. Dr. Ernesto G. Birgin (orientador) - IME-USP.

- Prof. Dr. Marcelo Gomes de Queiroz - IME-USP.

- Prof. Dr. Francisco de Assis Magalhães Gomes Neto - IMECC-UNICAMP. 

Dedico este trabalho ao meu pai, Antonio Carlos, e à minha mãe, Roseli. 



\section{Agradecimentos}

Agradeço ao meu pai e à minha mãe por todo o apoio que recebi durante toda a minha vida. Quaisquer palavras que eu usasse aqui não seriam suficientes para expressar todo o meu agradecimento.

Ao meu orientador, professor Ernesto Birgin, por sua atenção em todas as nossas reuniões, por sua motivação, por seu profundo interesse e dedicação a este trabalho.

Também agradeço aos professores Carlos Eduardo Ferreira, Marcelo Gomes de Queiroz e Francisco Gomes pelos comentários valiosos que contribuíram para o aperfeiçoamento desta dissertação.

Aos meus amigos do BCC 2003, pelas diversas partidas de boliche e de futebol no Cepê, e a todo o pessoal da "salinha", pela companhia e amizade ao longo desses anos. 


\section{Resumo}

Muitos problemas de otimização envolvem tanto variáveis inteiras quanto contínuas e podem ser modelados como problemas de programação não-linear inteira mista. Problemas dessa natureza aparecem com freqüência em engenharia química e incluem, por exemplo, síntese de processos, projeto de colunas de destilação, síntese de rede de trocadores de calor e produção de óleo e gás.

Neste trabalho, apresentamos algoritmos baseados em Lagrangianos Aumentados e branch and bound para resolver problemas de programação não-linear inteira mista. Duas abordagens são consideradas. Na primeira delas, um algoritmo do tipo Lagrangianos Aumentados é usado como método para resolver os problemas de programação não-linear que aparecem em cada um dos nós do método branch and bound. Na segunda abordagem, usamos o branch and bound para resolver os problemas de minimização em caixas com variáveis inteiras que aparecem como subproblemas do método de Lagrangianos Aumentados. Ambos os algoritmos garantem encontrar a solução ótima de problemas convexos e oferecem recursos apropriados para serem usados na resolução de problemas não convexos, apesar de não haver garantia de otimalidade nesse caso.

Apresentamos um problema de empacotamento de retângulos em regiões convexas arbitrárias e propomos modelos para esse problema que resultam em programas nãolineares com variáveis inteiras e contínuas. Realizamos alguns experimentos numéricos e comparamos os resultados obtidos pelo método descrito neste trabalho com os resultados alcançados por outros métodos.

Também realizamos experimentos com problemas de programação não-linear inteira mista encontrados na literatura e comparamos o desempenho do nosso método ao de outro disponível publicamente.

Palavras-chave: programação não-linear, programação não-linear inteira mista, Lagrangianos Aumentados, variáveis inteiras. 


\section{Abstract}

Many optimization problems contain both integer and continuous variables and can be modeled as mixed-integer nonlinear programming problems. Problems of this nature appear frequently in chemical engineering and include, for instance, process synthesis, design of distillation columns, heat exchanger network synthesis and oil and gas production.

In this work, we present algorithms based on Augmented Lagrangians and branch and bound for solving mixed-integer nonlinear programming problems. Two approaches are considered. In the first one, an Augmented Lagrangian algorithm is used for solving nonlinear programming problems that appear at each node in the branch and bound method. In the second approach, we use a branch and bound method for solving boxconstrained problems with integer variables that appear as subproblems of the Augmented Lagrangian algorithm. Both algorithms guarantee to find an optimal solution for convex problems and have appropriate strategies to deal with non-convex problems, although there is no guarantee of optimality in this case.

We present a problem of packing rectangles within an arbitrary convex region and propose models for this problem that result in nonlinear programs with integer and continuous variables. We have performed some numerical experiments and compared the results reached by the method described in this work and the results obtained by other methods.

We have also performed experiments with mixed-integer nonlinear programming problems found in the literature and compared the performance of our method to that of other method publicly available.

Keywords: nonlinear programming, mixed-integer nonlinear programming, Augmented Lagrangian, integer variables. 


\section{Sumário}

Sumário vii

Lista de Figuras $\quad$ ix

Lista de Tabelas $\quad$ xi

1 Introdução 1

2 Branch and Bound $\quad \mathbf{5}$

2.1 Descrição do método . . . . . . . . . . . . . . . . 6

2.1 .1 Avaliação . . . . . . . . . . . . . . . . . . 6

2.1 .2 Exploração . . . . . . . . . . . . . . . . . 6 6

2.1 .3 Divisão . . . . . . . . . . . . . . . . . . . . . 7

2.2 Seleção da variável de divisão . . . . . . . . . . . . . . . . . . . . . . 10

2.2 .1 Pseudo-custos . . . . . . . . . . . . . . . . . 10

2.3 Escolha do subproblema a ser resolvido . . . . . . . . . . . . . 13

3 Lagrangianos Aumentados $\quad 15$

3.1 Descrição do método . . . . . . . . . . . . . . . . . . . . 16

3.2 Lagrangianos Aumentados com variáveis inteiras e contínuas . . . . . . . . 17

3.2.1 Branch and bound com o método de Lagrangianos Aumentados para as relaxações . . . . . . . . . . . . . . . . . 18

3.2.2 Branch and bound para minimização em caixas com variáveis inteiras 18

3.2 .3 Otimalidade do método . . . . . . . . . . . . . . . . . . . 19

3.2.4 Estratégias para problemas não-convexos . . . . . . . . . . . . . 21

3.3 Experimentos computacionais . . . . . . . . . . . . . . . . . 22

4 Detalhes de implementação $\quad 31$

4.1 Número inteiro em ponto flutuante . . . . . . . . . . . . . . . . . . . . . . . . 31

4.2 Arredondamento de soluções . . . . . . . . . . . . . . . . . . . . . 32

4.3 Pontos iniciais . . . . . . . . . . . . . . . . . . . 33 
5 Problemas de empacotamento $\quad 35$

5.1 Empacotamento ortogonal de retângulos . . . . . . . . . . . . . . 36

5.2 Empacotamento ortogonal com rotação . . . . . . . . . . . . . . . 39

5.2 .1 Primeiro modelo . . . . . . . . . . . . . . . . . 40

5.2 .2 Um modelo mais adequado . . . . . . . . . . . . . . . 45

5.2 .3 Ângulo de rotação . . . . . . . . . . . . . . . . . . . . . . . . . . . . . 47

5.2 .4 Ponto inicial . . . . . . . . . . . . . . . . . . . . . 49

5.3 Experimentos computacionais . . . . . . . . . . . . . . . 53

5.3 .1 Ângulo de rotação . . . . . . . . . . . . . . . . . . 56

5.3 .2 Ponto inicial . . . . . . . . . . . . . . . . . . 57

5.3 .3 Instâncias 12 '-16' . . . . . . . . . . . . . . . . . . 6 62

5.3 .4 Fixando as variáveis inteiras . . . . . . . . . . . . . . . 63

5.3.5 Modelo de empacotamento com restrições . . . . . . . . . . . . . 63

5.3 .6 Relaxando o corte . . . . . . . . . . . . . . . . . . . . . 64

5.3 .7 Modelo em AMPL . . . . . . . . . . . . . . . . . . . 68

5.3 .8 Representações gráficas das soluções . . . . . . . . . . . . . . 68

6 Considerações finais e trabalhos futuros $\quad 71$

$\begin{array}{ll}\text { A Convexidade } & \mathbf{7 3}\end{array}$

A.1 Definição . . . . . . . . . . . . . . . . . . . . . . . 73

A.2 Convexidade da função Lagrangiano Aumentado . . . . . . . . . . . . . 74

$\begin{array}{ll}\text { B Problemas } & 77\end{array}$

$\begin{array}{lr}\text { Referências Bibliográficas } & 85\end{array}$ 


\section{Lista de Figuras}

2.1 (a) Divisão de um problema em dois subproblemas através da fixação do valor da variável $y_{i}$ em 0 no primeiro subproblema e em 1 no segundo. (b) Dois novos subproblemas criados impondo-se um limite superior na variável $y_{i}$ de $\left\lfloor\hat{y}_{i}\right\rfloor$ em um deles e um limite inferior de $\left\lceil\hat{y}_{i}\right\rceil$ no outro. . . . . . . . 8

$2.2 k$ subproblemas gerados fixando-se, em cada um deles, o valor da variável $y_{i}$ em um dos possíveis valores do conjunto discreto $Y_{i} \ldots \ldots . \ldots 8$

2.3 Divisão em uma restrição de limite superior generalizado. . . . . . . . . . . 9

3.1 Perfil de desempenho de IAlgencan e Algencan-Bb. . . . . . . . . . . 28

3.2 Perfil de desempenho dos métodos IAlgencan e Bonmin. . . . . . . . . 29

4.1 Exemplo do procedimento de arredondamento que resulta em um ponto inviável. A solução do problema relaxado é $y_{r}$. Arredondando essa solução, obtemos $y_{a}$, um ponto inviável do problema principal. . . . . . . . . . . 32

5.1 (a) Solução para o problema tratado em [18]. (b) Solução para o problema intermediário introduzido neste capítulo. (c) Solução para o problema abordado em [17]. . . . . . . . . . . . . . . . . . . . . . . 36

5.2 Vértices e centro do retângulo $i$ com dimensões $a^{i}$ e $b^{i} \ldots \ldots \ldots$. . . . . 36

5.3 Representação gráfica de uma solução para o problema ORP em que $m=$ $33, a=0.9, b=0.3$ e $\Omega=\left\{x \in \mathbb{R}^{2} \mid x_{1}^{2}-x_{2} \leq 0,-x_{1}+x_{2}^{2}-6 x_{2}+6 \leq\right.$

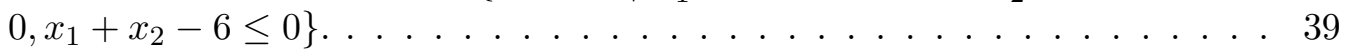

5.4 Exemplo de solução para o problema de empacotamento ortogonal com rotação. Note que os retângulos estão rotacionados em relação ao eixo cartesiano, mas estão posicionados ortogonalmente entre si. . . . . . . . . . 39

5.5 Método para determinar se dois retângulos estão sobrepostos. (a) Posicionamento dos retângulos. (b) Os retângulos rotacionados de $-\theta$ e a fácil verificação de sobreposição. . . . . . . . . . . . . . . . . . . . . . 44 41

5.6 Ilustração do procedimento de determinação dos vértices dos retângulos. (a) Os centros dos retângulos com seus vértices ainda desconhecidos. (b) "Desrotação" dos retângulos seguida do cálculo trivial dos vértices $\hat{V}_{d}^{i, \theta}$. (c) Rotação dos vértices dos retângulos e fim do procedimento. . . . . . . . . . 42

5.7 Para $\theta \in[0, \pi]$, é possível obter qualquer rotação de forma independente do

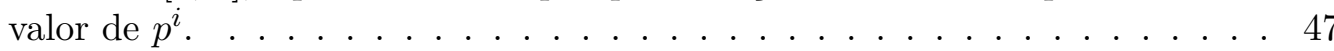


5.8 Possíveis posições do retângulo $i$ considerando $\theta \in[0, \pi / 2]$. Em (a), o caso em que $p^{i}=0$, e, em (b), o caso em que $p^{i}=1 \ldots \ldots$. . . . . . 48

5.9 Exemplo da introdução de pontos estacionários com a mudança nos limites da variável $\theta$. Em (a), temos um problema com quatro pontos estacionários. Em (b), temos um problema com apenas três pontos estacionários. . . . . . 48

5.10 Exemplo de cobertura de um retângulo por círculos. . . . . . . . . . . . . 50

5.11 Distribuição dos círculos dentro do retângulo com seus centros dados por

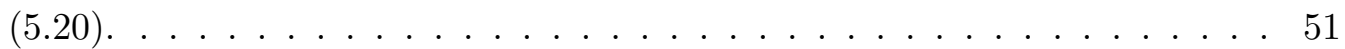

5.12 Distribuição dos círculos dentro do retângulo com seus centros dados por

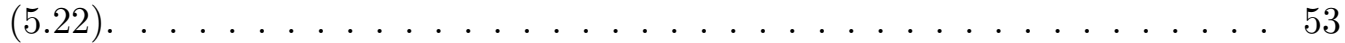

5.13 Soluções encontradas para a instância 8. Em (a), a solução encontrada pelo método que considera rotações ortogonais [18] e, em (c), a solução encontrada pelo método que considera rotações arbitrárias [17] (ambas com 32 retângulos). Em (b), a solução encontrada pelo nosso método com 33 retângulos. . . . . . . . . . . . . . . . . . . . 69

5.14 Representação gráfica das soluções encontradas para as instâncias 12'-16'. . 69

5.15 Representação gráfica das soluções encontradas para as instâncias 1-16. . 70 


\section{Lista de Tabelas}

3.1 Descrição dos problemas da MINLPLib. . . . . . . . . . . . . . 26

3.2 Desempenho do Algencan-Bb para os dezessete problemas de [31]. . . . . 27

3.3 Desempenho do IAlgencan na resolução dos dezessete problemas de [31] . 27

3.4 Desempenho dos métodos IAlgencan e Algencan-BB nos problemas da MINLPLib (Tabela 3.1) . . . . . . . . . . . . . . . . . . 28

3.5 Desempenho dos métodos IAlgencan e Bonmin nos 110 problemas selecionados da MINLPLib. . . . . . . . . . . . . . . . . . . 28

3.6 Comparação entre IAlgencAn e Bonmin. . . . . . . . . . . . . . . . . . . . 29

5.1 Definição dos problemas. . . . . . . . . . . . . . . . . 55

5.2 Resultados obtidos pelos métodos descritos em [17] e [18] . . . . . . . . 56

5.3 Resultados obtidos usando o modelo (5.17) implementado em Fortran, restringindo a variável $\theta$ ao intervalo $[0, \pi / 2]$ e usando a estratégia de reinícios com 5 resoluções por nó. . . . . . . . . . . . . . . . . . . . . 58

5.4 Resultados obtidos usando o modelo (5.17) implementado em Fortran, restringindo a variável $\theta$ ao intervalo $[0, \pi / 2]$ e usando a estratégia de reinícios com 100 resoluções por nó. . . . . . . . . . . . . . . . . . 58

5.5 Resultados obtidos usando o modelo (5.17) implementado em Fortran, restringindo a variável $\theta$ ao intervalo $[0, \pi]$ e usando a estratégia de reinícios com 5 resoluções por nó. . . . . . . . . . . . . . . . . . . 59

5.6 Resultados obtidos usando o modelo (5.17) implementado em Fortran, restringindo a variável $\theta$ ao intervalo $[0, \pi]$ e usando a estratégia de reinícios com 100 resoluções por nó. . . . . . . . . . . . . . . . . . . . 59

5.7 Resultados obtidos usando o modelo (5.17) implementado em Fortran, deixando livre a variável $\theta$ e usando a estratégia de reinícios com 5 resoluções por nó. . . . . . . . . . . . . . . . . . 6 60

5.8 Resultados obtidos usando o modelo (5.17) implementado em Fortran, deixando livre a variável $\theta$ e usando a estratégia de reinícios com 100 resoluções por nó. . . . . . . . . . . . . . . . . 6 60

5.9 Pontos iniciais gerados dentro da região convexa do problema. . . . . . . . . 61

5.10 Pontos iniciais dados pelas soluções do problema de empacotamento de círculos e usando a estratégia de reinícios com 5 resoluções por nó. . . . . 62 
5.11 Resultados obtidos pelos métodos descritos em [17] e [18] para os problemas 12'-16', e pelo nosso método com o modelo (5.17) implementado em Fortran e usando reinícios com 5 resoluções por nó. . . . . . . . . . . . . . . 63

5.12 Resultados obtidos usando reinícios com 100 resoluções por nó e resolvendo o subproblema novamente com as variáveis inteiras fixadas. . . . . . . . . 64

5.13 Modelo (5.18) com restrições, implementado em Fortran e usando reinícios com 5 resoluções por nó. . . . . . . . . . . . . . . . . . . 65

5.14 Modelo (5.18) com restrições, implementado em Fortran e usando reinícios com 100 resoluções por nó. . . . . . . . . . . . . . . . . . . . 65

5.15 Resultados obtidos usando o modelo (5.17) implementado em Fortran, deixando livre a variável $\theta$, usando a estratégia de reinícios com 5 resoluções por nó, $\alpha=10^{-4}$ e $M=0 . \ldots \ldots \ldots$. . . . . . . . . . 66

5.16 Resultados obtidos usando o modelo (5.17) implementado em Fortran, deixando livre a variável $\theta$, usando a estratégia de reinícios com 100 resoluções por nó, $\alpha=10^{-4}$ e $M=0 \ldots \ldots$. . . . . . . . . . . . 67

5.17 Resultados obtidos usando o modelo (5.17) implementado em Fortran, deixando livre a variável $\theta$, usando a estratégia de reinícios com 100 resoluções por nó, $\alpha=10^{-4}$ e $M=0$, e resolvendo o subproblema com as variáveis inteiras fixadas. . . . . . . . . . . . . . . . . . 6 67

5.18 Resultados obtidos usando o modelo (5.17) implementado em AMPL usando reinícios com 100 tentativas por nó. . . . . . . . . . . . . . . 68 


\section{Capítulo 1}

\section{Introdução}

Programação não-linear inteira mista (MINLP - Mixed Integer Nonlinear Programming) refere-se aos problemas de otimização que envolvem tanto variáveis inteiras quanto contínuas, bem como não-linearidades na função objetivo e nas restrições. Dizemos que uma variável é contínua se ela pode assumir valores reais. Uma variável é dita inteira se ela pode assumir apenas valores inteiros e discreta se ela assume valores em um conjunto discreto. Para o caso particular em que uma variável inteira pode tomar valores sobre o conjunto $\{0,1\}$, dizemos que tal variável é binária.

Um problema de MINLP tem a seguinte forma:

$$
\begin{array}{ll}
\text { minimizar } & f(x, y) \\
\text { sujeita a } & h(x, y)=0 \\
& g(x, y) \leq 0 \\
& x \in X \\
& y \in Y \cap \mathbb{Z}^{n_{2}}
\end{array}
$$

onde $f: \mathbb{R}^{n} \rightarrow \mathbb{R}, h: \mathbb{R}^{n} \rightarrow \mathbb{R}^{m}, g: \mathbb{R}^{n} \rightarrow \mathbb{R}^{p}, X \subseteq \mathbb{R}^{n_{1}}, Y \subseteq \mathbb{R}^{n_{2}}$ e $n=n_{1}+n_{2}$.

MINLP é amplamente utilizada na prática e problemas dessa natureza aparecem comumente em engenharia química (síntese de processos $[3,4,5,26,44]$, produção de óleo e gás [56], design, synthesis and scheduling of multipurpose batch plants [32, 47], síntese de rede de trocadores de calor [50], molecular design [62, 66], projeto de colunas de destilação [49]), minimização de desperdício em cortes de papel [33, 40, 52, 55], empacotamento [18], marketing de produtos [26, 37], finanças [58], entre outras áreas.

Algumas técnicas empregadas na resolução de problemas MINLPs são: Branch and Bound (Beale [10], Gupta e Ravindran [51], Ostrovsky et al. [63], Quesada e Grossmann [46], Fletcher e Leyffer [30], Tawarmalani e Sahinidis [68]), Decomposição de Benders Generalizada (Geoffrion [39], Floudas et al. [6]), Aproximação Externa (Duran e Grossmann [26], Grossmann e Kocis [43], Grossmann e Viswanathan [48]) e Aproximação Externa Generalizada (Fletcher e Leyffer [29]). Essas técnicas geralmente lidam com a resolução de sucessivos subproblemas (problemas aproximados) de programação não-linear. O branch and bound, por exemplo, envolve a resolução de problemas de programação nãolinear, que são relaxações do problema original obtidas, por exemplo, através da remoção das restrições de integralidade das variáveis inteiras. 
Problemas de programação não-linear inteira mista são, em geral, muito difíceis de serem resolvidos. Dois fatores principais contribuem para a dificuldade de resolução de problemas da forma (1.1): (i) a não-convexidade dos problemas, que possibilita a presença de múltiplas soluções locais, e (ii) a natureza combinatória dos problemas promovida pela presença de variáveis inteiras.

O objetivo deste trabalho é estender a aplicabilidade do Algencan $[7,8,13,16]$ a problemas de programação não-linear com variáveis inteiras e contínuas. ALGENCAN é um método de Lagrangianos Aumentados para resolução de problemas de programação nãolinear com restrições gerais. O método é completamente descrito em [7] e está disponível na $w e b^{1}$ como parte do Projeto Tango.

Uma primeira idéia é desenvolver um algoritmo do tipo branch and bound no qual, em cada nó, se resolva um problema de programação não-linear usando o AlGEnCAN. Nesse caso, contudo, poderíamos substituir o ALGENCAN por qualquer outro algoritmo que seja capaz de resolver problemas de programação não-linear. Alternativamente, consideramos um algoritmo do tipo Lagrangianos Aumentados que resolve em cada passo um subproblema com restrições de caixa e variáveis inteiras usando um algoritmo baseado em branch and bound.

Em sua forma mais simples, o algoritmo de Lagrangianos Aumentados implementado pelo Algencan consiste no seguinte processo: constrói-se a função Lagrangiano Aumentado penalizando-se todas as restrições do problema com exceção das restrições de caixa, que permanecem no nível inferior do programa. Dessa forma, obtém-se um subproblema que consiste na minimização de uma função não-linear com restrições de caixas. Esse subproblema é então resolvido por um algoritmo apropriado para problemas de minimização em caixas (como, por exemplo, Gencan [15]). Uma vez que esse subproblema é resolvido, os parâmetros de penalidade e a aproximação dos multiplicadores de Lagrange são atualizados, um novo subproblema é construído e o algoritmo prossegue desta maneira.

A idéia é modificar o algoritmo interno (que resolve os subproblemas de minimização em caixas) de forma que ele seja capaz de lidar com variáveis inteiras. Optamos por implementar um algoritmo do tipo branch and bound para tratar das restrições de integralidade que, em cada nó, resolva um problema de minimização em caixas (usando GENCAN, por exemplo).

O algoritmo proposto é capaz de encontrar a solução ótima de problemas de programação não-linear inteira mista cujas relaxações contínuas são problemas convexos. A relaxação contínua do problema (1.1), por exemplo, é o problema

$$
\begin{array}{ll}
\text { minimizar } & f(x, y) \\
\text { sujeita a } & h(x, y)=0 \\
& g(x, y) \leq 0 \\
& x \in X \\
& y \in Y
\end{array}
$$

em que as restrições de integralidade são removidas da variável inteira $y$. Contudo, o algoritmo proposto também pode ser usado para resolver problemas não-convexos sem a garantia de otimalidade global da solução encontrada.

\footnotetext{
${ }^{1}$ http://www.ime.usp.br/ egbirgin/tango/
} 
Este trabalho está organizado da seguinte forma. No Capítulo 2, apresentamos um algoritmo do tipo branch and bound para resolver problemas de programação não-linear inteira mista. No Capítulo 3, descrevemos o método de Lagrangianos Aumentados implementado pelo AlgEnCAN, e, na Seção 3.2, apresentamos e discutimos as duas abordagens consideradas para o desenvolvimento do algoritmo para MINLPs. Na Seção 3.3, apresentamos alguns resultados obtidos pelo algoritmo implementado com problemas encontrados na literatura. No Capítulo 4, expomos alguns detalhes relacionados à implementação do algoritmo. No Capítulo 5, apresentamos um problema de empacotamento de retângulos em regiões convexas e propomos dois modelos para esse problema. O primeiro deles resulta em um programa com função não-linear e restrições de caixas, além de restrições de integralidade, e o segundo modelo resulta em um programa não-linear com restrições de desigualdade e variáveis inteiras e contínuas. Realizamos alguns testes e comparamos os resultados alcançados pelo nosso método com resultados obtidos por outros métodos. Finalmente, no Capítulo 6, tecemos algumas conclusões e discutimos os trabalhos ainda a serem realizados. 


\section{Capítulo 2}

\section{Branch and Bound}

Branch and bound é uma técnica de otimização que utiliza uma árvore de enumeração para resolver um problema. Cada nó desta árvore representa um subproblema do problema principal e cada ramo representa uma nova restrição a ser considerada. Um método branch and bound consiste principalmente de três processos: (i) dividir o problema em subproblemas mais simples (divisão), (ii) calcular limitantes inferior e superior para o valor ótimo do subproblema (avaliação), a fim de avaliar a possibilidade de encontrar uma solução melhor no ramo deste subproblema, e (iii) cortar ramos da árvore que não precisam ser visitados (exploração).

O método branch and bound foi introduzido por Land e Doig [57] em 1960. Desde então, foi e ainda é bastante utilizado como base para resolver muitos problemas de otimização. Particularmente, problemas de programação linear e não-linear inteira mista têm sido resolvidos com relativo sucesso. Podemos citar, por exemplo, Beale [10], Benichou et al. [12], Gauthier e Ribière [36] e Mitra [60] para aplicações de branch and bound na resolução de problemas de programação linear inteira mista. Na resolução de problemas de programação não-linear inteira mista, podemos citar, por exemplo, Bonami et al. [19], Eckstein [27], Gupta e Ravindran [51], Quesada e Grossmann [46], Ostrovsky et al. [63] e Fletcher e Leyffer [30].

Neste capítulo apresentamos um algoritmo baseado no método branch and bound para resolver problemas de programação não-linear inteira mista.

Definição 2.1 Dizemos que $P_{s}$ é um subproblema de um problema $P$ se $P_{s}$ tem a mesma função objetivo e se o seu conjunto viável está contido no conjunto viável de $P$.

Em nosso caso, especificamente, associaremos o método a uma árvore da seguinte forma: cada nó da árvore representará um problema de programação não-linear inteira mista, sendo a raiz o problema principal, e cada aresta representará a adição de uma nova restrição (no caso mais simples, uma restrição de limite inferior, superior ou de igualdade em uma variável). Assim, cada nó herdará o problema do nó pai (isto é, do nó antecessor) com uma restrição a mais. 


\subsection{Descrição do método}

O método mantém a melhor solução $s$ conhecida para o problema, cujo valor $z$ começa com $+\infty$, e um conjunto de problemas candidatos (problemas ainda não visitados) que começa contendo apenas o problema principal. Enquanto houver problemas candidatos, seleciona-se e remove-se um problema $P$ desse conjunto. Seja $P^{\prime}$ o problema que se obtém a partir de $P$ através da relaxação das restrições de integralidade. Dessa forma, $P^{\prime}$ é um problema de programação não-linear padrão. Se a solução de $P^{\prime}$ satisfizer as restrições de integralidade, então ela é uma solução ótima do problema $P$. Nesse caso, comparamos o valor $z_{P}$ dessa solução com o valor $z$ da melhor solução conhecida até o momento. Se $z_{P}<z$, então uma solução melhor do que a anterior foi encontrada e $s$, assim como o seu correspondente valor $z$, são atualizados.

Se a solução de $P^{\prime}$ não satisfizer as restrições de integralidade, existe uma variável, digamos $y_{i}$, que viola uma dessas restrições. Tal variável é chamada de variável de divisão (branching variable). P é então dividido em pelo menos dois subproblemas. Cada um dos subproblemas criados é incorporado ao conjunto de problemas candidatos e o método segue resolvendo o próximo subproblema, que deve ser selecionado dentre aqueles pertencentes ao conjunto de problemas candidatos. Após todos os subproblemas terem sido resolvidos/explorados, a solução ótima é a melhor solução descoberta durante o processo.

\subsubsection{Avaliação}

$\mathrm{Na}$ tentativa de resolver um problema, normalmente é feita uma relaxação desse problema. Seja $P^{\prime}$ uma relaxação para um problema $P$.

Definição 2.2 Um problema de otimização $P^{\prime}$ é definido como uma relaxação do problema $P$ se o conjunto de soluções viáveis de $P$ é um subconjunto das soluções viáveis de $P^{\prime}$.

Sendo assim, se $P^{\prime}$ não tem solução viável, então $P$ também não tem. Além disso, se $z_{P}$ é o valor da solução ótima do problema $P$ e $z_{P^{\prime}}$ é o valor da solução ótima do problema $P^{\prime}$, então $z_{P^{\prime}} \leq z_{P}$. Ou seja, a solução do problema relaxado fornece um limitante inferior para a solução do problema original. Mais ainda, se uma solução ótima de $P^{\prime}$ é solução viável de $P$, então ela é uma solução ótima de $P$.

Um problema relaxado pode ser obtido, por exemplo, através da remoção das restrições de integralidade do problema original, transformando-o em um problema de programação não-linear padrão. Chamaremos esse tipo de relaxação de relaxação contínua. No entanto, existe uma relação a ser considerada envolvendo o modo de relaxação e a qualidade do limitante: quanto mais fácil de resolver o problema relaxado mais distante o limitante inferior obtido poderá estar da solução do problema original.

\subsubsection{Exploração}

$\mathrm{Na}$ análise dos subproblemas candidatos, é necessário determinar quais devem ser examinados em detalhe e quais podem ser descartados. Um subproblema candidato $P$ será considerado explorado se (i) pudermos garantir que o conjunto de soluções viáveis de 
$P$ não contém uma solução melhor do que a melhor solução encontrada até o momento ou se (ii) uma solução ótima de $P$ for encontrada. Em ambos os casos, o subproblema candidato $P$ pode ser considerado resolvido e não precisa ser examinado em mais detalhes através de uma nova divisão. Considerando uma relaxação $P^{\prime}$ de $P$, existem basicamente três situações que permitem considerar um subproblema $P$ explorado. São elas:

(i) Inviabilidade. $P^{\prime}$ não possui solução viável. Nesse caso, $P$ também não possuirá solução viável e ele pode ser descartado da busca.

(ii) Eliminação por avaliação. O limitante inferior de $P$ é maior ou igual ao valor da melhor solução viável obtida até o momento. (Em particular, quando a solução ótima de $P^{\prime}$ tem valor maior ou igual ao da melhor solução encontrada até o momento.) Nesse caso não há possibilidade de encontrar uma solução melhor do que a atual e o subproblema $P$ pode ser descartado da busca.

(iii) Otimalidade. A solução ótima de $P^{\prime}$ é solução viável de $P$ e, portanto, é solução ótima de $P$.

Note que a solução da relaxação do problema pai de $P$ fornece um limitante inferior para $P$. Assim, a resolução de $P^{\prime}$ pode ser evitada no caso em que a solução da relaxação do problema pai de $P$ tiver um valor maior ou igual ao da melhor solução encontrada até o momento.

\subsubsection{Divisão}

O processo de divisão dá-se quando, através dos critérios apresentados na Seção 2.1.2, não for possível cortar o ramo correspondente ao problema em questão. A idéia nesta etapa é utilizar a estratégia de divisão e conquista para resolver o problema. Apresentaremos aqui as formas mais comuns de divisão.

Considere o conjunto de soluções viáveis de um problema $P$. O problema será dividido em pelo menos dois subproblemas de modo que qualquer solução viável de um subproblema seja uma solução viável de $P$ e cada solução viável de $P$ seja solução viável de exatamente um de seus subproblemas. Nesse caso, os conjuntos viáveis dos subproblemas formam uma partição do conjunto viável de $P$.

Definição 2.3 $S_{S}=\left\{S_{1}, \ldots, S_{k}\right\}$ é dita uma separação de um conjunto $S$, onde $S_{i} \subseteq S$, $i=1 \ldots k$, se

$$
\bigcup_{S_{i} \in S_{S}}=S
$$

Definição 2.4 $S_{P}=\left\{S_{1}, \ldots, S_{k}\right\}$ é dita uma partição de um conjunto $S$ se $S_{P}$ é uma separação de $S$ e

$$
S_{i} \cap S_{j}=\emptyset
$$

para $i, j=1, \ldots, k$ e $i \neq j$. 
Seja $y_{i}$ uma variável que viola uma restrição de integralidade e que foi escolhida como variável de divisão. Seja $\hat{y}_{i}$ o valor de $y_{i}$ na solução do problema relaxado $P^{\prime}$. Se $y_{i}$ for uma variável binária, serão criados dois subproblemas. No primeiro, o valor da variável $y_{i}$ será fixado em 0 e no segundo seu valor será fixado em 1 . Se $y_{i}$ for uma variável inteira, também serão criados dois subproblemas. A restrição $y_{i} \leq\left\lfloor\hat{y}_{i}\right\rfloor$ será adicionada no primeiro subproblema e a restrição $y_{i} \geq\left\lceil\hat{y}_{i}\right\rceil$ será inserida no segundo subproblema, onde $\lfloor b\rfloor$ denota o maior número inteiro menor ou igual a $b$. A Figura 2.1 mostra esses dois casos.

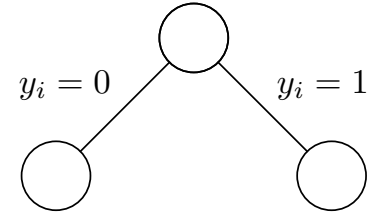

(a)

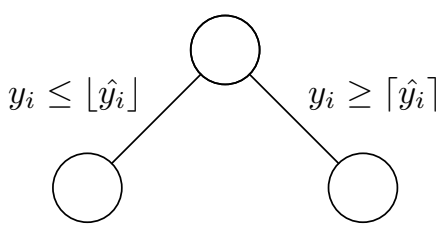

(b)

Figura 2.1: (a) Divisão de um problema em dois subproblemas através da fixação do valor da variável $y_{i}$ em 0 no primeiro subproblema e em 1 no segundo. (b) Dois novos subproblemas criados impondo-se um limite superior na variável $y_{i}$ de $\left\lfloor\hat{y}_{i}\right\rfloor$ em um deles e um limite inferior de $\left\lceil\hat{y}_{i}\right\rceil$ no outro.

No caso em que $y_{i}$ for uma variável discreta, temos uma outra possibilidade. Podemos criar tantos subproblemas quantos forem os valores do conjunto ao qual $y_{i}$ deve pertencer. Em cada um deles, a variável $y_{i}$ terá seu valor fixado em um dos possíveis valores para $y_{i}$. Suponha que $Y_{i}=\left\{v_{1}, v_{2}, \ldots, v_{k}\right\}$ seja o domínio da variável $y_{i}$ (com $v_{1}<v_{2}<\ldots<v_{k}$ ). A Figura 2.2 ilustra essa situação. Alternativamente, podemos escolher um índice $j$ tal que $1 \leq j \leq k-1$ e criar apenas dois subproblemas. Em um deles adicionamos a restrição $y_{i} \leq v_{j}$ e no outro adicionamos a restrição $y_{i} \geq v_{j+1}$.

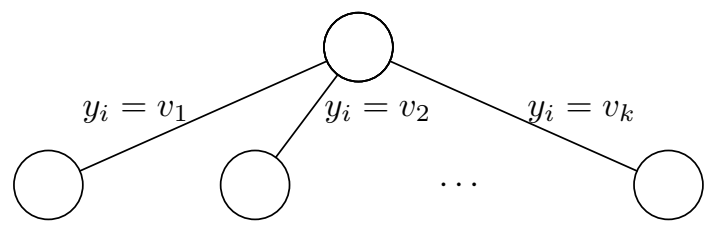

Figura 2.2: $k$ subproblemas gerados fixando-se, em cada um deles, o valor da variável $y_{i}$ em um dos possíveis valores do conjunto discreto $Y_{i}$.

Note que a solução de $P^{\prime}$ é inviável em todos os subproblemas de $P$ e seu valor é um limitante inferior para eles. 


\section{Limite superior generalizado}

Um outro tipo de divisão pode ser usado quando temos uma restrição de limite superior generalizado (GUB - Generalized Upper Bound) da forma

$$
\sum_{j \in T} y_{j}=1 \quad \text { ou } \quad \sum_{j \in T} y_{j} \leq 1
$$

para algum $T \subseteq\left\{1, \ldots, n_{2}\right\}$ e $y_{j} \in\{0,1\}$ para $j \in T$.

Considere uma variável $y_{i}$ tal que $i \in T$ e $y_{i}$ não satisfaz as restrições de integralidade em uma dada solução. De acordo com a divisão convencional, poderíamos criar dois subproblemas com a imposição da restrição $y_{i}=0$ no primeiro e $y_{i}=1$ no segundo. No primeiro subproblema, teríamos no máximo $|T|$ possibilidades de valores para $y_{j} \mathrm{com}$ $j \in T \backslash\{i\}$ (considerando o GUB com igualdade, para cada $j \in T \backslash\{i\}$ poderíamos ter $y_{j}=1$ e $y_{k}=0$ para $k \in T \backslash\{j\}$, totalizando $|T|-1$ possibilidades; considerando o GUB com desigualdade, teríamos uma possibilidade a mais, que seria $y_{j}=0$ para todo $j \in T)$, enquanto que no segundo subproblema teríamos apenas uma $\left(y_{j}=0\right.$ para todo $j \in T \backslash\{i\})$. Assim, o ramo criado pela restrição $y_{i}=0$ poderia ter um conjunto viável de soluções muito maior do que o ramo criado pela restrição $y_{i}=1$, de forma que a árvore de busca ficaria desbalanceada nesse sentido. Além disso, é possível que pouco progresso seja feito com essa divisão dada a semelhança dos conjuntos viáveis do primeiro subproblema e de seu problema pai.

Note que, devido à restrição $(2.1)$, a restrição $y_{i}=1$ é equivalente à restrição

$$
\sum_{j \in T \backslash\{i\}} y_{j}=0
$$

O balanceamento da árvore pode ser melhorado se considerarmos uma generalização desse tipo de divisão. Podemos escolher um subconjunto $T_{1}$ de $T$ tal que

$$
0<\sum_{j \in T_{1}} \hat{y}_{j}<1
$$

na solução do subproblema e criar dois subproblemas ao impor a restrição $\sum_{j \in T_{1}} y_{j}=0$ no primeiro subproblema e a restrição $\sum_{j \in T \backslash T_{1}} y_{j}=0$ no segundo. Essa divisão foi introduzida por Beale e Tomlin [11].

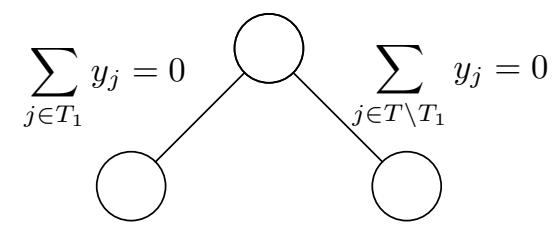

Figura 2.3: Divisão em uma restrição de limite superior generalizado. 


\subsection{Seleção da variável de divisão}

Para a escolha da variável de divisão, uma opção simples é selecionar a variável de menor índice que não satisfaz sua restrição de integralidade. Essa regra de certa forma arbitrária pode ser usada para derivar uma outra um pouco mais complexa, que leva em consideração a importância de cada uma das variáveis [51]. A idéia é dar prioridade às variáveis consideradas mais importantes, que podem ser definidas com base em informações fornecidas pelo modelo do problema. Considerando mais importantes as variáveis de menor índice, basta fazer uma reordenação dos índices das variáveis e usar a regra de seleção da variável de menor índice para se levar em conta essa prioridade.

Uma outra opção é escolher a variável mais "fracionária" [20, 51], isto é, a variável que maximiza o valor $\min \left\{\hat{y}_{i}-\left\lfloor\hat{y}_{i}\right\rfloor,\left\lceil\hat{y}_{i}\right\rceil-\hat{y}_{i}\right\}$, onde $\hat{y}_{i}$ denota o valor da variável $y_{i}$. Em outras palavras, é a variável que possui o valor mais longe de um número inteiro. A idéia por trás dessa estratégia é tentar provocar a maior degradação da função objetivo com a esperança de que os ramos que surgem a partir desta divisão possam ser cortados.

\subsubsection{Pseudo-custos}

O conceito de pseudo-custos foi introduzido por Benichou et al. [12] dentro do contexto de programação linear inteira mista. Pseudo-custos são medidas que têm por objetivo quantificar a importância de uma variável e prever a deterioração da função objetivo quando esta variável fica restrita a um determinado intervalo. Ainda que sua justificativa seja baseada apenas em experimentos, pseudo-custos são bastante utilizados no momento de decidir qual variável será usada para se dividir um problema $[1,2,19,27,36,41,42,59]$.

Apresentamos, a seguir, a definição de pseudo-custos dada por Benichou et al. [12]. Em seguida, mostramos alguns métodos utilizados para o cálculo dos pseudo-custos. Por fim, apresentamos algumas regras para a escolha de variáveis de divisão com base em pseudo-custos.

\section{Definição}

A cada variável inteira $y_{i}$, são associados dois valores: o pseudo-custo inferior desta variável, denotado por $P C_{i}^{-}$, e o pseudo-custo superior, denotado por $P C_{i}^{+}$. O primeiro tenta prever o acréscimo provocado na função objetivo, por unidade de mudança da variável $y_{i}$, quando esta variável tem seu valor forçado para baixo de um determinado valor inteiro. O segundo tenta estimar o acréscimo da função objetivo, por unidade de mudança de $y_{i}$, quando esta variável tem seu valor forçado para cima de um determinado valor inteiro.

Considere um problema $P$ e uma solução $s_{R}$ para a relaxação contínua de $P$. Seja $f_{P}$ o valor da solução $s_{R}$. Suponha que $s_{R}$ não satisfaça as restrições de integralidade, isto é, que exista uma variável $y_{i}$ cujo valor $\hat{y}_{i}$ na solução $s_{R}$ não é inteiro. Considere, agora, o problema $P^{-}$que se obtém a partir de $P$ quando impomos a restrição $y_{i} \leq\left\lfloor\hat{y}_{i}\right\rfloor$ e o problema $P^{+}$que se obtém a partir de $P$ quando impomos a restrição $y_{i} \geq\left\lceil\hat{y}_{i}\right\rceil$. Sejam $f_{P^{-}}$e $f_{P^{+}}$os valores das soluções das relaxações contínuas de $P^{-}$e $P^{+}$, respectivamente. 
Os pseudo-custos inferior $P C_{i}^{-}$e superior $P C_{i}^{+}$da variável $y_{i}$ são definidos como

$$
P C_{i}^{-}=\frac{f_{P^{-}}-f_{P}}{\hat{y}_{i}-\left\lfloor\hat{y}_{i}\right\rfloor} \quad \text { e } \quad P C_{i}^{+}=\frac{f_{P^{+}}-f_{P}}{\left\lceil\hat{y}_{i}\right\rceil-\hat{y}_{i}} .
$$

\section{Cálculo dos pseudo-custos}

Como é possível notar, os valores dos pseudo-custos de uma variável dependem do nó em que são computados. Além disso, esses valores não são conhecidos no início do algoritmo, a menos que o usuário seja capaz de fornecê-los. Sendo assim, é preciso definir como esses valores serão inicializados e atualizados ao longo da execução do algoritmo.

Em experimentos com problemas reais de programação linear inteira mista, Benichou et al. [12] calcularam os pseudo-custos de cada variável inteira em todos os nós da árvore em que ela não assumia um valor inteiro. Eles relataram que os pseudo-custos apresentaram a mesma ordem de grandeza nos diferentes nós em que foram calculados, com exceção de alguns poucos nós. Com base nesta observação, decidiram considerar constantes os pseudo-custos de cada variável inteira ao longo do algoritmo e fixá-los com os valores obtidos na primeira vez em que a variável é usada como variável de divisão.

Posteriormente, e dando seqüência ao trabalho realizado por Benichou et al. [12], Gauthier e Ribière [36] realizam mais experimentos e exploram o uso de pseudo-custos. Os pseudo-custos de uma variável inteira escolhida para ser a variável de divisão de um determinado subproblema são calculados assim que os seus dois subproblemas descendentes são resolvidos.

Gauthier e Ribière [36] levantam a questão de que, nesse caso, regras baseadas em pseudo-custos não seriam tão precisas no início do algoritmo, visto que muitas variáveis inteiras ainda não teriam seus pseudo-custos calculados. Para contornar esse possível inconveniente, Gauthier e Ribière [36] sugerem o seguinte procedimento: em um nó em que os pseudos-custos de uma variável são necessários mas não são conhecidos, um subproblema artificial é criado e resolvido, e sua solução é usada para calcular os pseudo-custos desta variável. Esses pseudo-custos são temporários e são substituídos pelos definitivos no momento em que o subproblema correspondente ao nó em questão é resolvido.

Eckstein [27] propõe tomar como valor para o pseudo-custo de uma variável a média de todas as observações dos pseudo-custos desta variável realizadas até então. Inicialmente, é atribuído o valor zero para o pseudo-custo de cada variável. À medida que os pseudocustos das variáveis são calculados, Eckstein [27] sugere atribuir os seguintes valores para o pseudo-custo de uma determinada variável:

1. se o seu pseudo-custo já foi calculado em algum momento, toma-se como valor a média de todos os pseudo-custos calculados para esta variável;

2. caso ele ainda não tenha sido calculado, toma-se como valor a média dos pseudocustos das demais variáveis.

Explicamos a seguir o procedimento com mais detalhes.

Seja $S_{i}^{-}$o conjunto de problemas $P$ para os quais a variável $y_{i}$ foi escolhida como variável de divisão e a relaxação contínua de $P^{-}$é viável e o valor $f_{P^{-}}$de sua solução 
é conhecido. Analogamente, definimos $S_{i}^{+}$. Para cada problema $P$, seja $v_{P}$ o valor da variável de divisão de $P$ na solução de $P^{\prime}$. Os pseudo-custos da variável $y_{i}$ são, então, dados por

$$
P C_{i}^{-}=\frac{1}{\left|S_{i}^{-}\right|} \sum_{P \in S_{i}^{-}} \frac{f_{P^{-}}-f_{P}}{v_{P}-\left\lfloor v_{P}\right\rfloor}
$$

e

$$
P C_{i}^{+}=\frac{1}{\left|S_{i}^{+}\right|} \sum_{P \in S_{i}^{+}} \frac{f_{P^{+}}-f_{P}}{\left\lceil v_{P}\right\rceil-v_{P}} .
$$

Caso $S_{i}^{-}$seja um conjunto vazio, isto é, $y_{i}$ ainda não foi escolhida como variável de divisão, então definimos seu pseudo-custo inferior como a média dos pseudo-custos inferiores de todas as variáveis que já fizeram o papel de variável de divisão. Seja $S^{-}$o conjunto de problemas $P$ para os quais o valor da solução da relaxação contínua de $P^{-}$é conhecida. Assim, o pseudo-custo inferior da variável $y_{i}$ será dado por

$$
P C_{i}^{-}=\frac{1}{\left|S^{-}\right|} \sum_{P \in S^{-}} \frac{f_{P^{-}}-f_{P}}{v_{P}-\left\lfloor v_{P}\right\rfloor} .
$$

De forma análoga, definimos $S^{+}$e o pseudo-custo superior de $y_{i}$ será dado por

$$
P C_{i}^{+}=\frac{1}{\left|S^{+}\right|} \sum_{P \in S^{+}} \frac{f_{P^{+}}-f_{P}}{\left\lceil v_{P}\right\rceil-v_{P}}
$$

se $S_{i}^{+}$estiver vazio. Se $S^{-}$estiver vazio, então $P C_{i}^{-}$é inicializado com valor 0 , assim como $\mathrm{PC}_{i}^{+}$, caso $S^{+}$esteja vazio. Resumindo, os pseudo-custos inferior e superior de $y_{i}$ são dados respectivamente por

$$
P C_{i}^{-}= \begin{cases}\frac{1}{\left|S_{i}^{-}\right|} \sum_{P \in S_{i}^{-}} \frac{f_{P^{-}}-f_{P}}{v_{P}-\left\lfloor v_{P}\right\rfloor} & \text { se } S_{i}^{-} \neq \emptyset \\ \frac{1}{\left|S^{-}\right|} \sum_{P \in S^{-}} \frac{f_{P^{-}}-f_{P}}{v_{P}-\left\lfloor v_{P}\right\rfloor} & \text { se } S_{i}^{-}=\emptyset \text { e } S^{-} \neq \emptyset \\ 0 & \text { caso contrário, }\end{cases}
$$

$\mathrm{e}$

$$
P C_{i}^{+}= \begin{cases}\frac{1}{\left|S_{i}^{+}\right|} \sum_{P \in S_{i}^{+}} \frac{f_{P^{+}}-f_{P}}{\left\lceil v_{P}\right\rceil-v_{P}} & \text { se } S_{i}^{+} \neq \emptyset \\ \frac{1}{\left|S^{+}\right|} \sum_{P \in S^{+}} \frac{f_{P^{+}}-f_{P}}{\left\lceil v_{P}\right\rceil-v_{P}} & \text { se } S_{i}^{+}=\emptyset \text { se } S^{+} \neq \emptyset \\ 0 & \text { caso contrário. }\end{cases}
$$

\section{Critérios para a seleção da variável de divisão}

Sejam $D_{i}^{-}=P C_{i}^{-}\left(\hat{y}_{i}-\left\lfloor\hat{y}_{i}\right\rfloor\right)$ e $D_{i}^{+}=P C_{i}^{+}\left(\left\lceil\hat{y}_{i}\right\rceil-\hat{y}_{i}\right)$. Benichou et al. [12] propõem escolher como variável de divisão a variável de índice $i^{*}$ tal que

$$
i^{*}=\arg \max _{i} \min \left\{D_{i}^{-}, D_{i}^{+}\right\} .
$$


A justificativa é a de que a degradação esperada da função objetivo seria maior com a divisão sendo realizada na variável $i^{*}$, de forma que os nós provenientes dessa divisão teriam uma chance maior de serem eliminados através de uma possível violação do limitante superior.

Benichou et al. [12] também consideram atribuir prioridades às variáveis e selecionar, em cada nó, a variável mais importante, de acordo com a prioridade previamente estabelecida, que não satisfaça as restrições de integralidade. Eles sugerem organizar as variáveis em ordem decrescente do valor

$$
\max \left\{P C_{i}^{-}, P C_{i}^{+}\right\}
$$

Essa ordenação é fixa desde o início e os valores de $P C_{i}^{-}$e $P C_{i}^{+}$são dados por experimentos anteriores com o mesmo problema. Benichou et al. [12] realizaram testes para mostrar a influência de uma boa e de uma má ordenação das variáveis. Dois tipos de ordenação são investigados: o primeiro em que a ordem das variáveis é dada por valor decrescente de $\max \left\{P C_{i}^{-}, P C_{i}^{+}\right\}$, o que corresponde a selecionar a variável $i^{*}$ (com valor não inteiro) tal que

$$
i^{*}=\arg \max _{i} \max \left\{P C_{i}^{-}, P C_{i}^{+}\right\}
$$

e a segunda através da ordenação inversa das variáveis, o que significa selecionar a variável $i^{*}$ tal que

$$
i^{*}=\arg \min _{i} \max \left\{P C_{i}^{-}, P C_{i}^{+}\right\} .
$$

Os resultados mostram que o segundo tipo de ordenação foi muito pior do que o primeiro, exigindo a resolução de um número muito maior de subproblemas. Benichou et al. [12] também argumentam que, apesar da ordenação das variáveis não ter uma relação direta com a descoberta de uma solução inteira ótima, ela pode ser determinante no momento de provar a otimalidade de uma solução inteira já encontrada.

Eckstein [27] apresenta um critério mais geral para a seleção de variáveis, que leva em conta tanto a prioridade das variáveis, definida inicialmente, quanto os valores de seus pseudo-custos. Sua sugestão é escolher a variável $i^{*}$ tal que

$$
i^{*}=\arg \max _{i} \alpha_{0} Q_{i}+\alpha_{1} \min \left\{d_{i}^{-}, d_{i}^{+}\right\}+\alpha_{2} \min \left\{D_{i}^{-}, D_{i}^{+}\right\}+\alpha_{3} \max \left\{D_{i}^{-}, D_{i}^{+}\right\},
$$

onde $Q_{i}$ é a prioridade atribuída à variável de índice $i, d_{i}^{-}=\hat{y}_{i}-\left\lfloor\hat{y}_{i}\right\rfloor, d_{i}^{+}=\left\lceil\hat{y}_{i}\right\rceil-\hat{y}_{i} \mathrm{e}$ $\alpha_{0}, \alpha_{1}, \alpha_{2}$ e $\alpha_{3}$ são parâmetros escalares a serem escolhidos pelo usuário. De acordo com Eckstein [27], $\alpha_{0}$ deveria idealmente assumir um valor relativamente grande, de forma a dar prioridade às variáveis consideradas mais importantes. Em seus experimentos, Eckstein [27] não leva em consideração as prioridades das variáveis (por falta desses dados) e define $\alpha_{1}=1, \alpha_{2}=10$ e $\alpha_{3}=1$.

\subsection{Escolha do subproblema a ser resolvido}

Uma estratégia simples é escolher o último subproblema gerado pelo algoritmo. Tendo em mente uma lista de subproblemas a serem resolvidos, essa estratégia é conhecida como "último a entrar, primeiro a sair" (last in, first out-LIFO) e seu uso corresponde a fazer 
uma busca em profundidade na árvore. Note que, quanto mais profundo na árvore um subproblema estiver localizado, mais restrições nas variáveis ele apresentará. Com isso, espera-se encontrar uma solução viável (e, conseqüentemente, obter um limitante superior) rapidamente, que será útil para cortar ramos subseqüentes da árvore.

Outra estratégia, menos usual mas igualmente simples, é a busca em largura. Essa estratégia considera todos os subproblemas de um mesmo nível antes de considerar os subproblemas do próximo nível. Os subproblemas gerados são inseridos no final da lista e o próximo subproblema a ser resolvido é o que está localizado no início da lista. Tal estratégia também é conhecida como "primeiro a entrar, último a sair" (first in, first out - FIFO).

Uma outra estratégia que deve ser mencionada é chamada de "regra do melhor limitante" (best bound rule [51, 61]), que seleciona o subproblema com o menor limitante inferior. Uma justificativa para essa escolha vem do fato de que nenhum subproblema que possui limitante inferior estritamente maior do que o valor da solução ótima do problema principal precisará passar pela etapa de divisão do método branch and bound. Além disso, o subproblema com o menor limitante inferior é o único que fornece um limitante inferior para o problema principal. Portanto, elevando-se o menor limitante inferior, obtemos informações acerca do valor da solução global do problema principal e da distância à melhor solução encontrada até então.

Em [42] podem ser encontradas outras estratégias de seleção de subproblemas. 


\section{Capítulo 3}

\section{Lagrangianos Aumentados}

Apresentamos, neste capítulo, uma breve descrição do método de Lagrangianos Aumentados introduzido em [7, 8]. Esse método foi desenvolvido para resolver problemas de programação não-linear com restrições gerais e uma de suas motivações vem da possibilidade de tirar proveito da existência de métodos eficientes para a resolução de subproblemas com restrições parciais (isto é, subproblemas que apresentam um subconjunto do conjunto de restrições do problema principal). Uma implementação prática, chamada de ALGENCAN, encontra-se disponível publicamente na $w e b^{1}$ como parte do Projeto TANGo.

Considere o seguinte problema de otimização:

$$
\begin{array}{ll}
\text { minimizar } & f(x) \\
\text { sujeita a } & x \in \Omega .
\end{array}
$$

O conjunto de restrições $\Omega$ pode ser visto como um conjunto da forma $\Omega=\Omega_{1} \cap \Omega_{2}$, onde o conjunto de restrições $\Omega_{2}$ é composto por restrições consideradas "fáceis". Por restrições fáceis, entendemos que problemas da forma

$$
\begin{array}{ll}
\text { minimizar } & F(x) \\
\text { sujeita a } & x \in \Omega_{2}
\end{array}
$$

são mais fáceis de se resolver do que os problemas da forma (3.1). Isso quer dizer que algoritmos apropriados e eficientes para minimização local de (3.2) são conhecidos, mas tais algoritmos não podem ser aplicados diretamente na resolução de (3.1). A idéia do método de Lagrangianos Aumentados é resolver iterativamente o problema (3.1) tirando proveito dos métodos capazes de resolver os problemas da forma (3.2). Uma iteração do método de Lagrangianos Aumentados consiste em resolver um subproblema da forma (3.2) e em seguida atualizar os parâmetros de penalidade e as aproximações dos multiplicadores de Lagrange, como veremos a diante.

Na Seção 3.1, explicamos breve e simplificadamente o funcionamento do método de Lagrangianos Aumentados. Em seguida, na Seção 3.2, apresentamos dois métodos baseados em Lagrangianos Aumentados e branch and bound para a resolução de problemas de programação não-linear com variáveis inteiras e contínuas. O primeiro deles, descrito

\footnotetext{
${ }^{1}$ http://www . ime.usp.br/ egbirgin/tango/
} 
na Seção 3.2.1, combina branch and bound e o método de Lagrangianos Aumentados, de forma que as relaxações em cada nó da árvore do método branch and bound são resolvidas pelo método de Lagrangianos Aumentados. Na Seção 3.2.2, discutimos a abordagem que considera as restrições de integralidade como restrições "simples" e utiliza o método branch and bound na resolução dos subproblemas presentes nas iterações do método de Lagrangianos Aumentados. A otimalidade desses dois métodos é tratada na Seção 3.2.3. Na Seção 3.2.4, discutimos algumas estratégias para tornar o método mais robusto quando usado na resolução de problemas não-convexos. Por fim, na Seção 3.3, apresentamos alguns experimentos computacionais.

\subsection{Descrição do método}

Consideremos $\Omega_{1}=\left\{x \in \mathbb{R}^{n} \mid h_{1}(x)=0, g_{1}(x) \leq 0\right\}$ e $\Omega_{2}=\left\{x \in \mathbb{R}^{n} \mid h_{2}(x)=\right.$ $\left.0, g_{2}(x) \leq 0\right\}$, onde $f: \mathbb{R}^{n} \rightarrow \mathbb{R}, h_{1}: \mathbb{R}^{n} \rightarrow \mathbb{R}^{m_{1}}, g_{1}: \mathbb{R}^{n} \rightarrow \mathbb{R}^{p_{1}}, h_{2}: \mathbb{R}^{n} \rightarrow \mathbb{R}^{m_{2}} \mathrm{e}$ $g_{2}: \mathbb{R}^{n} \rightarrow \mathbb{R}^{p_{2}}$ são contínuas e diferenciáveis. Assim, estamos interessados em resolver o seguinte problema:

$$
\begin{array}{ll}
\operatorname{minimizar} & f(x) \\
\text { sujeita a } & h_{1}(x)=0 \\
& g_{1}(x) \leq 0 \\
& h_{2}(x)=0 \\
& g_{2}(x) \leq 0 .
\end{array}
$$

O Lagrangiano Aumentado de Powell-Hestenes-Rockafellar $[54,64,65]$ em relação a $\Omega_{1}$ para esse problema é dado por

$$
L_{\rho}(x, \lambda, \mu)=f(x)+\frac{\rho}{2}\left\{\sum_{i=1}^{m_{1}}\left[\left[h_{1}(x)\right]_{i}+\frac{\lambda_{i}}{\rho}\right]^{2}+\sum_{i=1}^{p_{1}}\left[\max \left(0,\left[g_{1}(x)\right]_{i}+\frac{\mu_{i}}{\rho}\right)\right]^{2}\right\}
$$

para todo $x \in \mathbb{R}^{n}, \rho>0, \lambda \in \mathbb{R}^{m_{1}}$ e $\mu \in \mathbb{R}_{+}^{p_{1}}$. Em (3.4), $\rho$ é o parâmetro de penalidade, $\lambda_{i}$, $i=1, \ldots, m_{1}$, são as aproximações dos multiplicadores de Lagrange associados às restrições de igualdade e $\mu_{i}, i=1, \ldots, p_{1}$ são as aproximações dos multiplicadores de Lagrange associados às restrições de desigualdade. O método de Lagrangianos Aumentados consiste, basicamente, de sucessivas minimizações de $L_{\rho}(x, \lambda, \mu)$ sobre o conjunto $\Omega_{2}$, seguidas de atualizações adequadas de $\lambda, \mu$ e $\rho$. Podemos resumir simplificadamente o algoritmo da seguinte forma.

\section{Algoritmo 3.1}

Sejam $\lambda_{\min }<\lambda_{\max }, \mu_{\max }>0, \gamma>1,0<\tau<1, \lambda_{i}^{1} \in\left[\lambda_{\min }, \lambda_{\max }\right], i=1, \ldots, m$, $\mu_{i}^{1} \in\left[0, \mu_{\max }\right], i=1, \ldots, p$ e $\rho_{1}>0$.

Passo 1. Resolução do subproblema.

Calcule $x^{k} \in \mathbb{R}^{n}$ uma solução aproximada de

$$
\begin{array}{ll}
\text { minimizar } & L_{\rho_{k}}\left(x, \lambda_{k}, \mu_{k}\right) \\
\text { sujeita a } & x \in \Omega_{2} .
\end{array}
$$


Passo 2. Defina

$$
V_{i}^{k}=\max \left\{\left[g_{1}\left(x^{k}\right)\right]_{i},-\frac{\mu_{i}^{k}}{\rho_{k}}\right\}, i=1, \ldots, p
$$

Se $k=1$ ou

$$
\max \left\{\left\|h_{1}\left(x^{k}\right)\right\|_{\infty},\left\|V^{k}\right\|_{\infty}\right\} \leq \tau \max \left\{\left\|h_{1}\left(x^{k-1}\right)\right\|_{\infty},\left\|V^{k-1}\right\|_{\infty}\right\}
$$

defina $\rho_{k+1}=\rho_{k}$. Senão, defina $\rho_{k+1}=\gamma \rho_{k}$.

Passo 3. Calcule $\lambda_{i}^{k+1} \in\left[\lambda_{\min }, \lambda_{\max }\right]$ para $i=1, \ldots, m$ e $\mu_{i}^{k+1} \in\left[0, \mu_{\max }\right]$ para $i=$ $1, \ldots, p$. Faça $k \leftarrow k+1$ e vá para o Passo 1 .

O Algenchn é uma implementação prática desse método para o caso em que $\Omega_{2}$ é composto apenas por restrições de caixa, isto é, $\Omega_{2}=\left\{x \in \mathbb{R}^{n} \mid l \leq x \leq u\right\}$, onde $l, u \in \mathbb{R}^{n}$. Nesse caso, o problema (3.5) no Passo 1 do Algoritmo 3.1 torna-se um problema de minimização em caixas. Para resolver esse problema, podemos usar, por exemplo, um algoritmo como Genche [15] ou Betra [9], que são métodos de restrições ativas para resolver problemas de minimização em caixas. Em implementações práticas do Algoritmo 3.1, também é comum aplicar a atualização de primeira ordem das aproximações dos multiplicadores, isto é, tomar

$$
\lambda_{i}^{k+1}=\mathcal{P}_{\left[\lambda_{\min }, \lambda_{\max }\right]}\left(\lambda_{i}^{k}+\rho_{k}\left[h_{1}\left(x^{k}\right)\right]_{i}\right)
$$

e

$$
\mu_{i}^{k+1}=\mathcal{P}_{\left[0, \mu_{\max }\right]}\left(\mu_{i}^{k}+\rho_{k}\left[g_{1}\left(x^{k}\right)\right]_{i}\right),
$$

onde $\mathcal{P}_{S}(z)$ denota a projeção euclidiana de $z$ no conjunto $S$.

\subsection{Lagrangianos Aumentados com variáveis inteiras e con- tínuas}

Estamos interessados em resolver problemas de programação não-linear com variáveis inteiras e contínuas. Como temos à nossa disposição um método capaz de resolver problemas de programação não-linear, a primeira idéia que surge é a de usar um método do tipo branch and bound para tratar das variáveis inteiras e usar o método de Lagrangianos Aumentados para resolver as relaxações em cada nó da árvore do método branch and bound. Nesse caso, o método de Lagrangianos Aumentados poderia ser substituído por qualquer outro método que resolva problemas de programação não-linear. Uma segunda possibilidade é estender o método de Lagrangianos Aumentados de forma que ele próprio seja capaz de lidar com as variáveis inteiras. Essas duas abordagens são descritas a seguir.

Introduziremos a variável inteira $y$ em nosso problema e vamos considerar daqui em diante $\Omega_{1}=\left\{(x, y) \in \mathbb{R}^{n} \mid h(x, y)=0, g(x, y) \leq 0\right\}$ e $\Omega_{2}=\left\{x \in X, y \in Y \cap \mathbb{Z}^{n_{2}}\right\}$, onde $f: \mathbb{R}^{n} \rightarrow \mathbb{R}, h: \mathbb{R}^{n} \rightarrow \mathbb{R}^{m}, g: \mathbb{R}^{n} \rightarrow \mathbb{R}^{p}$ são contínuas e diferenciáveis, $X \subset \mathbb{R}^{n_{1}} \mathrm{e}$ $Y \subset \mathbb{R}^{n_{2}}$. Consideraremos, também, $X$ e $Y$ compostos apenas por restrições de caixa, isto 
é, $X=\left\{x \in \mathbb{R}^{n_{1}} \mid l_{x} \leq x \leq u_{x}\right\}$ e $Y=\left\{y \in \mathbb{R}^{n_{2}} \mid l_{y} \leq y \leq u_{y}\right\}$. Desse modo, o problema em que estamos interessados em resolver toma a seguinte forma:

$$
\begin{array}{ll}
\text { minimizar } & f(x, y) \\
\text { sujeita a } & h(x, y)=0 \\
& g(x, y) \leq 0 \\
& x \in X \\
& y \in Y \cap \mathbb{Z}^{n_{2}} .
\end{array}
$$

\subsubsection{Branch and bound com o método de Lagrangianos Aumentados para as relaxações}

Considere um método do tipo branch and bound aplicado diretamente na resolução do problema (3.6). Neste caso, em cada nó da árvore do branch and bound, aparecem problemas de programação não-linear inteira mista que são subproblemas do problema principal que estamos interessados em resolver. Na etapa de avaliação desse método, como descrito na Seção 2.1.1, normalmente é necessário resolver relaxações desses subproblemas. Essas relaxações podem ser obtidas, por exemplo, através da remoção das restrições de integralidade das variáveis, de modo que tais relaxações tornam-se problemas de programação não-linear padrão. Considerando o problema (3.6), essas relaxações terão o seguinte formato:

$$
\begin{array}{ll}
\text { minimizar } & f(x, y) \\
\text { sujeita a } & h(x, y)=0 \\
& g(x, y) \leq 0 \\
& x \in X \\
& y \in \bar{Y} \subseteq Y,
\end{array}
$$

onde $\bar{Y}$ é uma restrição de caixa.

O problema (3.7) é um problema de programação não-linear padrão, de forma que podemos usar o método de Lagrangianos Aumentados para resolvê-lo. Sendo o método de Lagrangianos Aumentados descrito pelo Algoritmo 3.1 destinado a otimização local, um algoritmo do tipo branch and bound para resolver o problema (3.6) e que usa o Algoritmo 3.1 para resolver as relaxações do tipo (3.7) também será um algoritmo de otimização local.

Como o método branch and bound descrito no Capítulo 2 apóia-se no fato de que as relaxações em cada nó devem ser resolvidas de forma ótima, não temos garantia de encontrar a solução global do problema usando esta abordagem. Algumas estratégias para usadas para tentar melhorar a qualidade da solução encontrada serão discutidas adiante, na Seção 3.2.4.

\subsubsection{Branch and bound para minimização em caixas com variáveis in- teiras}

Consideremos agora uma abordagem diferente. Na sua forma mais simples, AlGENCAN considera as restrições de caixa como restrições do nível inferior e penaliza todas as outras 
restrições. Dessa forma, os subproblemas do Passo 1 do Algoritmo 3.1 são subproblemas de minimização em caixas.

Se, ao lidarmos com problemas do tipo (3.6), considerarmos as restrições de integralidade como restrições do nível inferior (juntamente com as restrições de caixa) então os subproblemas do Passo 1 do Algoritmo 3.1 serão problemas de minimização em caixas com restrições de integralidade nas variáveis, e terão a seguinte forma:

$$
\begin{array}{ll}
\operatorname{minimizar} & L_{\rho}(x, y, \lambda, \mu) \\
\text { sujeita a } & x \in X \\
& y \in Y \cap \mathbb{Z}^{n_{2}},
\end{array}
$$

onde

$$
L_{\rho}(x, y, \lambda, \mu)=f(x, y)+\frac{\rho}{2}\left\{\sum_{i=1}^{m}\left[[h(x, y)]_{i}+\frac{\lambda_{i}}{\rho}\right]^{2}+\sum_{i=1}^{p}\left[\max \left(0,[g(x, y)]_{i}+\frac{\mu_{i}}{\rho}\right)\right]^{2}\right\} .
$$

Surge então a possibilidade de resolver o problema (3.6) aplicando o Algoritimo 3.1 desde que saibamos como resolver problemas de minimização em caixas com restrições de integralidade nas variáveis. Uma maneira simples de lidar com tais problemas é definir um algoritmo do tipo branch and bound que resolva os subproblemas relaxados da forma

$$
\begin{array}{ll}
\operatorname{minimizar} & L_{\rho}(x, y, \lambda, \mu) \\
\text { sujeita a } & x \in X \\
& y \in \bar{Y} \subseteq Y
\end{array}
$$

de cada um dos nós utilizando um algoritmo para minimização em caixas como, por exemplo, Gencan ou Betra.

Nesta abordagem, aplicamos o Algoritmo 3.1 ao problema (3.6) e resolvemos os subproblemas do tipo (3.8) com uma combinação de branch and bound e GENCAN (ou BETRA). Esta abordagem se contrapõe à abordagem da subseção anterior na qual consideramos um algoritmo do tipo branch and bound que utilizava o método de Lagrangianos Aumentados na resolução dos subproblemas relaxados.

\subsubsection{Otimalidade do método}

Em [14], Birgin et al. propõem um algoritmo baseado em Lagrangianos Aumentados para otimização global de problemas de programação não-linear. A diferença entre o algoritmo proposto em [14] e o apresentado na Seção 3.1 está na resolução do subproblema (3.5). Quando o método descrito pelo Algoritmo 3.1 é aplicado a problemas de programação não-linear, é comum exigir que $x^{k}$ satisfaça aproximadamente as condições KKT do subproblema (3.5). Já o método proposto em [14] exige que a solução encontrada para esse subproblema seja um minimizador $\epsilon_{k}$-global de $L_{\rho_{k}}$ em $\Omega_{2}$, de acordo com a seguinte definição.

Definição 3.1 Um ponto $x^{*}$ é chamado de minimizador $\epsilon$-global de uma função $f$ em um conjunto $S$ se

$$
f\left(x^{*}\right) \leq f(x)+\epsilon
$$

para todo $x \in S$. 
Seja $\left\{\epsilon_{k}\right\}$ uma seqüência de números não-negativos tal que $\lim _{k \rightarrow \infty} \epsilon_{k}=\epsilon \geq 0$. No método descrito em [14], ao invés de se procurar por uma solução aproximada de (3.5) no Passo 1 do Algoritmo 3.1, deseja-se encontrar $x^{k} \in \Omega_{2}$ tal que

$$
L_{\rho_{k}}\left(x^{k}, \lambda_{k}, \mu_{k}\right) \leq L_{\rho_{k}}\left(x, \lambda_{k}, \mu_{k}\right)+\epsilon_{k}
$$

para todo $x \in \Omega_{2}$. O teorema a seguir, cuja prova pode ser encontrada em [14], garante que a solução encontrada pelo método para o problema (3.3) é um minimizador $\epsilon$-global desde que, em cada iteração do Algoritmo 3.1, um ponto $x^{k}$ satisfazendo (3.10) seja encontrado.

Teorema 3.1 Supondo que a seqüência $\left\{x^{k}\right\}$ gerada pelo Algoritmo 3.1 (com (3.10) no lugar de (3.5)) seja bem definida e admita um ponto limite $x^{*}$, então $x^{*}$ é um minimizador $\epsilon$-global do problema (3.3).

Queremos estender esse resultado para o caso em que são introduzidas variáveis inteiras no problema (3.3), isto é, quando temos um problema da forma (3.6). Notemos que tanto no método descrito na Seção 3.2.1 quanto no método descrito na Seção 3.2.2, é preciso resolver problemas de minimização em caixas. No primeiro método, esses problemas aparecem no Passo 1 do Algoritmo 3.1, que é usado para resolver problemas de programação não-linear em cada nó do método branch and bound. De acordo com o Teorema 3.1, se resolvermos o problema de minimização em caixas de forma ótima, então o Algoritmo 3.1 encontra a solução global do problema de programação não-linear e o método branch and bound, por sua vez, encontrará a solução global do problema de programação não-linear inteira mista. Já no segundo método, o problema de minimização em caixas aparece em cada nó do branch and bound quando este é usado para resolver o problema (3.8). O problema (3.8), por sua vez, aparece no Passo 1 da adaptação do Algoritmo 3.1 para problemas de programação não-linear inteira mista. Assim, se encontrarmos a solução ótima do problema de minimização em caixas, também encontraremos a solução ótima de (3.8) e, pelo Teorema 3.1, encontraremos a solução ótima do problema de programação não-linear inteira mista. Portanto, se pudermos resolver o problema (3.9) de forma ótima, ambos os métodos encontrarão a solução global do problema (3.6).

Consideremos, primeiramente, o caso em que $L_{\rho_{k}}\left(x, y, \lambda_{k}, \mu_{k}\right)$ é uma função convexa. Uma condição suficiente para isso é que $f$ e $g_{i}$ para $i=1, \ldots, p$ sejam funções convexas e $h_{i}$ para $i=1, \ldots, m$ sejam funções afins (a prova desta afirmação é dada no Apêndice A). Neste caso, o problema (3.9) é convexo, já que $X$ e $\bar{Y}$ são conjuntos convexos, e toda solução local de (3.9) também é uma solução global para esse problema. Mais ainda, como $L_{\rho_{k}}\left(x, y, \lambda_{k}, \mu_{k}\right)$ é diferenciável, todo ponto estacionário é um minimizador global. Em particular, o minimizador global do problema (3.9) é também um minimizador $\epsilon$-global.

Consideremos Gencan [15] como método para resolver os subproblemas (3.9). Como GENCAN gera uma seqüência em que todos os pontos de acumulação são pontos estacionários de primeira ordem, no caso em que o problema é convexo, a solução encontrada pelo GENCAN será um minimizador local e, conseqüentemente, um minimizador global. Assim, sendo GencAn capaz de encontrar o minimizador global de (3.9), o Teorema 3.1 garante que a solução global do problema (3.6) será encontrada pelo Algoritmo 3.1. 
Para o caso em que $L_{\rho_{k}}\left(x, y, \lambda_{k}, \mu_{k}\right)$ não é convexa, GENCAN não garante encontrar a solução global do problema (3.9) e, portanto, o Algoritmo 3.1 não garante encontrar a solução global do problema (3.6). Neste caso, precisamos usar técnicas específicas para lidar com problemas não-convexos.

\subsubsection{Estratégias para problemas não-convexos}

Para que o algoritmo branch and bound descrito na Seção 2 encontre a solução ótima do problema principal, é preciso que cada um dos subproblemas resolvidos seja resolvido de forma ótima. No entanto, se o problema não for convexo, o ALGENCAN não garante encontrar a solução global do problema. Normalmente o que ele encontra são pontos estacionários. O mesmo ocorre com GENCAN ou BETRA no caso da segunda abordagem.

Mesmo sem a garantia de otimalidade global, podemos usar ALGENCAN para resolver os subproblemas de programação não-linear na etapa de avaliação do branch and bound (no caso da primeira abordagem) e GENCAN ou BETRA para resolver os subproblemas de minimização em caixa (no caso da segunda abordagem). Dessa forma, teríamos métodos heurísticos para resolver problemas não-convexos.

Apesar do nosso principal interesse ser o de desenvolver um método que seja capaz de encontrar pontos estacionários de um MINLP, podemos aumentar a chance de encontrar soluções globais de problemas não-convexos usando as estratégias descritas a seguir.

\section{Reinícios}

Como ocorre com todos os métodos de otimização local, o ponto inicial pode ter grande influência na solução encontrada pelo algoritmo. Além disso, ainda precisamos lidar com os casos previstos na teoria nos quais AlgEnCAN não encontra uma solução viável. Podemos tentar contornar esses problemas de uma forma bem simples, através da utilização da estratégia de reinícios (multistart). Essa estratégia consiste em executar o algoritmo diversas vezes para resolver o mesmo problema, partindo de pontos distintos, uniformemente distribuídos sobre a região viável do problema, de forma a aumentar a chance de encontrar uma solução global. Essa estratégia pode ser aplicada juntamente com um dos procedimentos a seguir:

1. fixamos um número máximo de vezes que um mesmo subproblema será resolvido e guardamos a melhor solução entre todas as encontradas;

2. estabelecemos um número máximo de tentativas e nos contentamos com a primeira solução viável encontrada.

Caso uma solução viável não seja encontrada, declaramos o problema como inviável e prosseguimos com o próximo subproblema.

\section{Tolerância do corte}

Considere o método branch and bound. Seja $v_{m}$ o valor da melhor solução inteira encontrada até o momento e seja $v_{r}$ o valor da solução encontrada para o subproblema relaxado 
em um dado nó da árvore do método branch and bound. No caso da primeira abordagem, descrita na Seção 3.2.1, esse subproblema relaxado é um problema de programação não-linear padrão, enquanto que na segunda abordagem trata-se de um problema de minimização em caixas.

Conforme descrito na Seção 2.1.2, se $v_{r} \geq v_{m}$ então esse nó é descartado da busca pela solução do problema. Supondo que $v_{r}$ seja o valor de uma solução global para o subproblema relaxado, não estaríamos perdendo a solução ótima do problema original ao descartarmos esse nó.

No entanto, tratando-se de um problema não-convexo, a solução encontrada para o subproblema relaxado pode não ser uma solução global. Neste caso, o corte poderia fazer com que a solução ótima do problema original fosse perdida, bastando que ela esteja no ramo enraizado pelo nó em questão.

Sendo assim, propomos a seguinte relaxação no critério de corte dos nós. Seja $M$ um limitante inferior ${ }^{2}$ para o problema que está sendo resolvido pelo método branch and bound e seja $\alpha \in[0,1]$. Ao invés de descartarmos um nó sempre que $v_{r} \geq v_{m}$, propomos descartá-lo quando

$$
\alpha v_{r}+(1-\alpha) M \geq v_{m} .
$$

Se $\alpha=1$, então a condição (3.11) torna-se $v_{r} \geq v_{m}$, a mesma que tínhamos anteriormente. Se estivermos resolvendo um problema convexo ou então se confiarmos no método responsável por resolver os problemas relaxados, podemos usar $\alpha=1$. Se não confiarmos no método para resolver os subproblemas e não desejarmos fazer corte algum, podemos tomar $\alpha=0$, de forma que a condição (3.11) passa a ser $M \geq v_{m}$. Note que, neste caso, a menos que $v_{m}=M$, nenhum corte seria feito. Se $v_{m}=M$ então neste caso teríamos encontrado a solução ótima do subproblema e o método terminaria com uma prova de otimalidade da solução encontrada.

\subsection{Experimentos computacionais}

Apresentamos nesta seção os resultados dos experimentos realizados com os nossos métodos na resolução de alguns problemas conhecidos na literatura. Chamaremos de AlgencAN-BB a implementação do método descrito na Seção 3.2.1 e de IAlgEnCAN a implementação do método descrito na Seção 3.2.2.

Os problemas usados em nossos testes foram extraídos da MINLPLib [22] (uma coleção de modelos de programação não-linear inteira mista) e do livro [31]. Os modelos de alguns problemas de [31] podem ser vistos no Apêndice B. Tanto os modelos da MINLPLib quanto os modelos presentes em [31] estão disponíveis na web em http://www.gamsworld.org/ minlp/minlplib.htm e em http://titan.princeton.edu/TestProblems/, respectivamente. Alguns deles estão implementados em AMPL [34] (Modeling Language for Mathematical Programming) e outros em GAMS [21] (General Algebraic Modeling System).

\footnotetext{
${ }^{2}$ Em particular, se o problema que está sendo resolvido pelo branch and bound for um subproblema do algoritmo de Lagrangianos Aumentados, cabe destacar que um limitante inferior para a função objetivo do MINLP original levando-se em conta as restrições do nível inferior também é um limitante inferior para o subproblema.
} 
Como nossos métodos possuem uma interface que possibilita a resolução de problemas modelados em AMPL, os modelos originalmente descritos em GAMS foram convertidos para o formato AMPL por meio da ferramenta CONVERT [24], que faz parte do sistema GAMS.

Para auxiliar na comparação dos diferentes métodos e estratégias, optamos por usar a ferramenta conhecida como perfil de desempenho, introduzida em [25]. A medida de desempenho escolhida foi o tempo de processamento necessário para se resolver o problema. Para comparar a qualidade da solução encontrada, usamos o seguinte critério. Dadas duas soluções com valores respectivamente $f_{1}$ e $f_{2}$, dizemos que elas são equivalentes se

$$
f_{1} \leq-10^{20} \text { e } f_{2} \leq-10^{20}
$$

$\mathrm{ou}$

$$
\left|f_{1}-f_{2}\right| \leq \max \left\{10^{-10}, 10^{-6} \min \left\{\left|f_{1}\right|,\left|f_{2}\right|\right\}\right\}
$$

A equação (3.12) trata do caso em que o problema é ilimitado e quaisquer soluções com valores de função abaixo de $-10^{20}$ são consideradas equivalentes. Já a equação (3.13) diz que duas soluções são consideradas equivalentes se elas possuírem valores relativamente próximos.

Dizemos que, para um dado conjunto de problemas, um método A é mais eficiente do que um método B se o método A for capaz de resolver um número maior de problemas consumindo menos tempo. Também dizemos que um método A é mais robusto do que um método B para um dado conjunto de instâncias se A é capaz de resolver mais problemas do que B.

Nossos métodos foram implementados usando as linguagens Fortran 77, Fortran 95 e $\mathrm{C}$ e foram compilados com o GNU Compiler Collection (GCC) versão 4.2.1 (que inclui o compilador GNU Fortran). A opção - O3 foi adotada na compilação. Os testes foram executados em um computador 2.4GHz Intel Core2 Quad Q6600 com 4.0GB de memória RAM e Sistema Operacional Linux.

O objetivo do primeiro experimento é avaliar as diferentes estratégias de escolha da variável de divisão e de seleção do subproblema. Para a escolha da variável de divisão, incluiremos em nossos experimentos a estratégia baseada em pseudo-custo, a que seleciona a variável mais fracionária, a estratégia que escolhe aleatoriamente uma variável e por fim a estratégia que seleciona a variável de menor índice. Para a seleção do subproblemas, consideramos as três seguintes estratégias: busca em profundidade, regra do melhor limitante e busca em largura. Como precisamos avaliar o desempenho dessas estratégias tanto no Algencan-Bb quanto no IAlgencan, e como temos um total de doze combinações de estratégias possíveis (quatro para a escolha da variável de divisão e três para a escolha do subproblema), optamos por realizar tais experimentos com um conjunto reduzido de problemas. Vamos usar, assim, os dezessete problemas presentes em [31].

No segundo experimento, pretendemos avaliar o desempenho dos métodos na resolução de alguns problemas da MINLPLib. Selecionamos 110 problemas da MINLPLib cujas características podem ser vistas na Tabela 3.1. A última coluna dessa tabela mostra o valor da melhor solução inteira conhecida de acordo com os dados da MINLPLib. Os resultados obtidos no primeiro experimento serão usados para determinar a estratégia mais 
adequada (pelo menos para aquele conjunto de problemas) para cada um dos métodos e essa será a estratégia considerada no segundo experimento.

Finalmente, comparamos o desempenho do Algencan-BB, do IAlgencan e do BonMIN [19] nesse subconjunto de problemas da MINLPLib. Bonmin é um framework contendo diversos algoritmos para programação não-linear inteira mista. Optamos por usar o método B-BB do Bonmin, que é baseado em branch and bound e é considerado o algoritmo padrão pelos desenvolvedores do Bonmin. Utilizamos em nossos testes a versão 1.0.1 do Bonmin (a última versão disponível até o momento da finalização deste texto) obtida diretamente do projeto COIN-OR [23].

Em todos os experimentos do IAlgencan e Algencan-BB, usamos a estratégia de reinícios com resolução de 5 subproblemas por nó e optamos por não usar a relaxação do corte proposta na Seção 3.2.4, isto é, usamos $\alpha=1$. Também impomos um limite máximo de tempo de 3 horas para resolver cada problema tanto para os métodos IALGENCAN e Algencan-BB quanto para o Bonmin.

\begin{tabular}{|c|c|c|c|c|}
\hline \multirow{2}{*}{ Problema } & \multicolumn{3}{|c|}{ Número de } & \multirow{2}{*}{$\begin{array}{l}\text { Valor da melhor } \\
\text { solução inteira }\end{array}$} \\
\hline & variáveis & variáveis inteiras & restrições & \\
\hline ALAN & 9 & 4 & 8 & 2.925000 \\
\hline ВATCH & 47 & 24 & 74 & 285506.508200 \\
\hline BATCHDES & 20 & 9 & 20 & 167427.657100 \\
\hline DEB6 & 476 & 20 & 508 & 201.739300 \\
\hline DEB7 & 814 & 20 & 898 & 116.584600 \\
\hline DEB8 & 824 & 20 & 898 & 116.584600 \\
\hline DEB9 & 814 & 20 & 918 & 116.584600 \\
\hline DEB10 & 183 & 22 & 130 & 209.427800 \\
\hline DU-OPT & 21 & 13 & 10 & 3.556300 \\
\hline DU-OPT 5 & 21 & 13 & 10 & 8.073700 \\
\hline EG_ALL_S & 8 & 7 & 26 & 8.237700 \\
\hline EG_DISC_S & 8 & 4 & 26 & 5.760500 \\
\hline EG_DISC2_S & 8 & 3 & 26 & 5.642100 \\
\hline EG_INT_S & 8 & 3 & 26 & 6.453100 \\
\hline ELF & 55 & 24 & 39 & 0.191700 \\
\hline ENPRO48 & 154 & 92 & 215 & 187277.259400 \\
\hline FAC1 & 23 & 6 & 19 & 160912610.000000 \\
\hline FAC2 & 67 & 12 & 34 & 331837500.000000 \\
\hline FAC3 & 67 & 12 & 34 & 31982310.000000 \\
\hline FEEDTRAY & 98 & 7 & 92 & -13.406000 \\
\hline FEEDTRAY2 & 88 & 36 & 284 & 0.000000 \\
\hline FUEL & 16 & 3 & 16 & 8566.119000 \\
\hline GASTRANS & 107 & 21 & 150 & 89.085800 \\
\hline GBD & 5 & 3 & 5 & 2.200000 \\
\hline GEAR & 5 & 4 & 1 & 0.000000 \\
\hline GEAR2 & 29 & 24 & 5 & 0.000000 \\
\hline GEAR3 & 9 & 4 & 5 & 0.000000 \\
\hline GEAR4 & 7 & 4 & 2 & 1.643400 \\
\hline HMITTELMAN & 17 & 16 & 8 & 13.000000 \\
\hline JOHNALL & 195 & 190 & 193 & -224.730200 \\
\hline GKOCIS & 12 & 3 & 9 & -1.923100 \\
\hline LOP97ICX & 987 & 899 & 88 & 4326.147700 \\
\hline M3 & 27 & 6 & 44 & 37.800000 \\
\hline
\end{tabular}




\begin{tabular}{|c|c|c|c|c|}
\hline \multirow{2}{*}{ Problema } & \multicolumn{3}{|c|}{ Número de } & \multirow{2}{*}{$\begin{array}{l}\text { Valor da melhor } \\
\text { solução inteira }\end{array}$} \\
\hline & variáveis & variáveis inteiras & restrições & \\
\hline MEANVARX & 36 & 14 & 45 & 14.369200 \\
\hline NOUs1 & 51 & 2 & 44 & 1.567100 \\
\hline NOUS2 & 51 & 2 & 44 & 0.626000 \\
\hline NVS01 & 4 & 2 & 4 & 12.469700 \\
\hline NVSO2 & 9 & 5 & 4 & 5.984600 \\
\hline NVS03 & 3 & 2 & 3 & 16.000000 \\
\hline NVS04 & 3 & 2 & 1 & 0.720000 \\
\hline NVS05 & 9 & 2 & 10 & 5.470900 \\
\hline NVS06 & 3 & 2 & 1 & 1.770300 \\
\hline NVS07 & 4 & 3 & 3 & 4.000000 \\
\hline NVS08 & 4 & 2 & 4 & 23.449700 \\
\hline NVS09 & 11 & 10 & 1 & -43.134300 \\
\hline NVS10 & 3 & 2 & 3 & -310.800000 \\
\hline NVS11 & 4 & 3 & 4 & -431.000000 \\
\hline NVS12 & 5 & 4 & 5 & -481.200000 \\
\hline NVS13 & 6 & 5 & 6 & -585.200000 \\
\hline NVS14 & 9 & 5 & 4 & -40153.723700 \\
\hline NVS15 & 4 & 3 & 2 & 1.000000 \\
\hline NVS16 & 3 & 2 & 1 & 0.703100 \\
\hline NVS17 & 8 & 7 & 8 & -1100.400000 \\
\hline NVS18 & 7 & 6 & 7 & -778.400000 \\
\hline NVS19 & 9 & 8 & 9 & -1098.400000 \\
\hline NVS20 & 17 & 5 & 9 & 230.922200 \\
\hline NVS21 & 4 & 2 & 3 & -5.684800 \\
\hline NVS22 & 9 & 4 & 10 & 6.058200 \\
\hline NVS23 & 10 & 9 & 10 & -1125.200000 \\
\hline NVS24 & 11 & 10 & 11 & -1033.200000 \\
\hline OAER & 10 & 3 & 8 & -1.923100 \\
\hline PARALLEL & 206 & 25 & 116 & 924.295600 \\
\hline PROB02 & 7 & 6 & 9 & 112235.000000 \\
\hline PROB03 & 3 & 2 & 2 & 10.000000 \\
\hline PROB10 & 3 & 1 & 3 & 3.445500 \\
\hline PROCSEL & 11 & 3 & 8 & -1.923100 \\
\hline PUMP & 25 & 9 & 35 & 128893.741000 \\
\hline RAVEM & 113 & 54 & 187 & 269590.219300 \\
\hline RAVEMPB & 113 & 54 & 187 & 269590.219300 \\
\hline RISK2BPB & 464 & 14 & 581 & -55.876100 \\
\hline SEP1 & 30 & 2 & 32 & -510.081000 \\
\hline SPECTRA2 & 70 & 30 & 73 & 13.978300 \\
\hline SPRING & 18 & 12 & 9 & 0.846200 \\
\hline ST_E13 & 3 & 1 & 3 & 2.000000 \\
\hline ST_E14 & 12 & 4 & 14 & 4.579600 \\
\hline ST_E15 & 6 & 3 & 6 & 7.667200 \\
\hline ST_E27 & 5 & 2 & 7 & 2.000000 \\
\hline ST_E29 & 12 & 8 & 8 & -0.943500 \\
\hline ST_E31 & 113 & 24 & 136 & -2.000000 \\
\hline ST_E32 & 36 & 19 & 19 & -1.430400 \\
\hline ST_E35 & 33 & 7 & 40 & 64868.076800 \\
\hline ST_E36 & 3 & 1 & 3 & -246.000000 \\
\hline ST_E38 & 5 & 2 & 4 & 7197.727100 \\
\hline ST_E40 & 5 & 3 & 9 & 30.414200 \\
\hline
\end{tabular}




\begin{tabular}{|l|c|c|c|r|}
\hline \multirow{2}{*}{ Problema } & \multicolumn{3}{|c|}{ Número de } & Valor da melhor \\
\cline { 2 - 4 } & variáveis & variáveis inteiras & restrições & soluço inteira \\
\hline \hline ST_MIQP1 & 6 & 5 & 2 & 281.000000 \\
ST_MIQP2 & 5 & 4 & 4 & 2.000000 \\
ST_MIQP3 & 3 & 2 & 2 & -6.000000 \\
ST_MIQP4 & 7 & 3 & 5 & -4574.000000 \\
ST_MIQP5 & 8 & 2 & 14 & -333.888900 \\
ST_TEST1 & 6 & 5 & 2 & 0.000000 \\
ST_TEST2 & 7 & 6 & 3 & -9.250000 \\
ST_TEST3 & 14 & 13 & 11 & -7.000000 \\
ST_TEST4 & 7 & 6 & 6 & -7.000000 \\
ST_TEST5 & 11 & 10 & 12 & -110.000000 \\
ST_TEST6 & 11 & 10 & 6 & 471.000000 \\
ST_TEST8 & 25 & 24 & 21 & -29605.000000 \\
ST_TESTGR1 & 11 & 10 & 6 & -12.811600 \\
ST_TESTGR3 & 21 & 20 & 21 & -20.590000 \\
ST_TESTPH4 & 4 & 3 & 11 & -80.500000 \\
SYNHEAT & 57 & 12 & 65 & 154997.334900 \\
SYNTHES1 & 7 & 3 & 7 & 6.009800 \\
SYNTHES2 & 12 & 5 & 15 & 73.035300 \\
SYNTHES3 & 18 & 8 & 24 & 68.009700 \\
TLN2 & 9 & 8 & 13 & 5.300000 \\
TLS2 & 38 & 33 & 25 & 5.300000 \\
VAR_CON10 & 574 & 12 & 465 & 444.214000 \\
VAR_CON5 & 574 & 12 & 465 & 278.144900 \\
WATERX & 71 & 14 & 55 & 910.471100 \\
WINDFAC & 15 & 3 & 14 & 0.254500 \\
\hline
\end{tabular}

Tabela 3.1: Descrição dos problemas da MINLPLib.

Na Tabela 3.2, podemos observar o desempenho do ALGENCAN-BB nos problemas de [31] para as diferentes estratégias de escolha de variável de divisão e de seleção de subproblema. Podemos notar que a estratégia que usa a regra do melhor limitante para a seleção do subproblema e escolhe a variável mais fracionária como a variável de divisão foi a mais eficiente de todas, sendo mais rápida em $23.53 \%$ dos problemas. Além disso, ela também foi a mais robusta e resolveu $82.35 \%$ dos problemas.

Na Tabela 3.3, temos o desempenho do IALgencan nos dezessete problemas de [31] para as diversas estratégias consideradas. Observamos que, para qualquer que seja a estratégia para a escolha da variável de divisão, IALGENCAN foi igualmente robusto usando a busca em largura e a regra do melhor limitante, resolvendo todos os problemas. A regra do melhor limitante também foi a estratégia mais eficiente para o IALGENCAN.

A Figura 3.1 exibe o perfil de desempenho de IALGENCAN e ALGENCAN-BB na resolução dos 110 problemas selecionados da MINLPLib. Os dados apresentados são relativos ao IALGENCAN usando a regra do melhor limitante e escolha da variável de divisão de menor índice, e ao ALGENCAN-BB usando também a regra do melhor limitante e com a escolha da variável mais fracionária como variável de divisão. A Tabela 3.4 auxilia na comparação. Podemos observar na Figura 3.1 que IALGENCAN é não apenas mais eficiente do que o AlgENCAN-BB, mas também é mais robusto. De fato, IALGENCAN resolve $85.45 \%$ 


\begin{tabular}{|l|l|r|c|}
\hline \multicolumn{2}{|c|}{ Estratégia } & Eficiência & Robustez \\
\hline \hline \multirow{4}{*}{ Melhor limitante } & Pseudo-custos & $17.65 \%$ & $70.59 \%$ \\
& Mais fracionária & $23.53 \%$ & $82.35 \%$ \\
& Aleatória & $17.65 \%$ & $70.59 \%$ \\
& Menor índice & $17.65 \%$ & $64.71 \%$ \\
\hline \multirow{5}{*}{ Busca em profundidade } & Pseudo-custos & $11.76 \%$ & $70.59 \%$ \\
& Mais fracionária & $5.88 \%$ & $76.47 \%$ \\
& Aleatória & $5.88 \%$ & $76.47 \%$ \\
& Menor índice & $11.76 \%$ & $64.71 \%$ \\
\hline \multirow{5}{*}{ Busca em largura } & Pseudo-custos & $5.88 \%$ & $76.47 \%$ \\
& Mais fracionária & $0.00 \%$ & $76.47 \%$ \\
& Aleatória & $5.88 \%$ & $76.47 \%$ \\
& Menor índice & $0.00 \%$ & $70.59 \%$ \\
\hline
\end{tabular}

Tabela 3.2: Desempenho do AlgencAn-BB para os dezessete problemas de [31].

\begin{tabular}{|l|l|r|c|}
\hline \multicolumn{2}{|c|}{ Estratégia } & Eficiência & Robustez \\
\hline \hline \multirow{3}{*}{ Melhor limitante } & Pseudo-custos & $23.53 \%$ & $100.00 \%$ \\
& Mais fracionária & $23.53 \%$ & $100.00 \%$ \\
& Aleatória & $0.00 \%$ & $100.00 \%$ \\
& Menor índice & $35.29 \%$ & $100.00 \%$ \\
\hline \multirow{3}{*}{ Busca em profundidade } & Pseudo-custos & $11.76 \%$ & $94.12 \%$ \\
& Mais fracionária & $0.00 \%$ & $88.24 \%$ \\
& Aleatória & $0.00 \%$ & $94.12 \%$ \\
& Menor índice & $11.76 \%$ & $88.24 \%$ \\
\hline \multirow{3}{*}{ Busca em largura } & Pseudo-custos & $5.88 \%$ & $100.00 \%$ \\
& Mais fracionária & $5.88 \%$ & $100.00 \%$ \\
& Aleatória & $5.88 \%$ & $100.00 \%$ \\
& Menor índice & $11.76 \%$ & $100.00 \%$ \\
\hline
\end{tabular}

Tabela 3.3: Desempenho do IALgEnCAN na resolução dos dezessete problemas de [31]. 


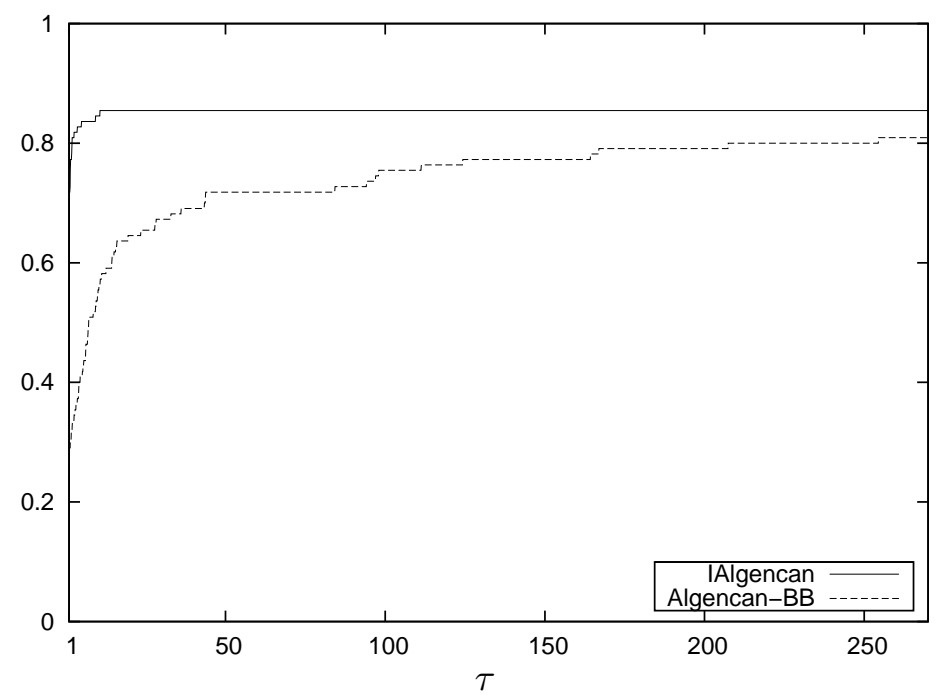

Figura 3.1: Perfil de desempenho de IAlgencan e Algencan-BB.

\begin{tabular}{|l|c|c|}
\hline Método & Eficiência & Robustez \\
\hline \hline IALGENCAN & $71.82 \%$ & $85.45 \%$ \\
ALGENCAN-BB & $23.64 \%$ & $80.91 \%$ \\
\hline
\end{tabular}

Tabela 3.4: Desempenho dos métodos IAlgencan e Algencan-BB nos problemas da MINLPLib (Tabela 3.1).

dos problemas, enquanto que AlgencAn-BB resolve 80.91\%. Além disso, IAlgencan é mais rápido em $71.82 \%$ dos problemas.

Sendo o desempenho do IAlgencan superior ao desempenho do AlgEnCAN-BB para esse conjunto de instâncias, comparamos agora o desempenho do IALGENCAN com o desempenho do Bonmin. A Figura 3.2 mostra o perfil de desempenho destes métodos e a Tabela 3.5 fornece um pouco mais de detalhe sobre os extremos do gráfico. Apesar do IAlgENCAN ser mais eficiente do que o Bonmin, este último é mais robusto. Enquanto que IALGENCAN foi capaz de resolver $80.91 \%$ dos problemas, o BonMIN conseguiu resolver $85.45 \%$.

Queremos saber, para cada um dos métodos, em quantos problemas o método encontra um ponto viável. Esses dados são apresentados na Tabela 3.6. Dos 110 problemas

\begin{tabular}{|l|c|c|}
\hline Método & Eficiência & Robustez \\
\hline \hline IALGENCAN & $50.91 \%$ & $80.91 \%$ \\
BONMIN & $46.36 \%$ & $85.45 \%$ \\
\hline
\end{tabular}

Tabela 3.5: Desempenho dos métodos IALgencan e Bonmin nos 110 problemas selecionados da MINLPLib. 


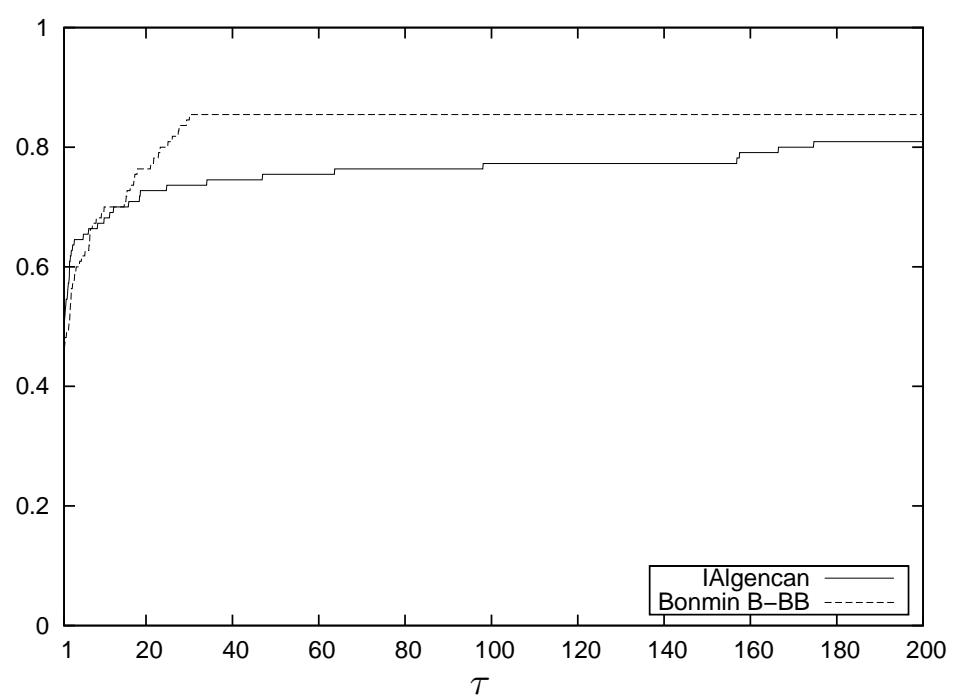

Figura 3.2: Perfil de desempenho dos métodos IAlgencan e Bonmin.

\begin{tabular}{|l|r|r|}
\cline { 2 - 3 } \multicolumn{1}{c|}{} & IALGENCAN & BONMIN \\
\hline Problemas viáveis & $102 / 110$ & $101 / 110$ \\
Satisfaz critérios de convergência & $102 / 102$ & $100 / 101$ \\
Melhor valor de função & $7 / 96$ & $13 / 96$ \\
Menor tempo (tolerância de 10\%) & $43 / 76$ & $33 / 76$ \\
\hline
\end{tabular}

Tabela 3.6: Comparação entre IALgencAn e Bonmin.

selecionados da MINLPLib, IALGENCAN encontra um ponto viável em 102 problemas, ao passo que Bonmin encontra em 101 problemas. Dos problemas em que IALGENCAN encontra um ponto viável, os critérios de convergência são satisfeitos em todos eles. Já para o BonMIN, o critério de convergência não é satisfeito para o problema LOP97ICX e o método pára por atingir o limite máximo de tempo. Dos 96 problemas em que ambos os métodos encontram uma solução viável, em 7 problemas a solução dada pelo IALGENCAN possui um valor melhor do que a encontrada pelo Bonmin, e em 13 problemas a solução dada pelo Bonmin é melhor do que a solução dada pelo IAlgencan. Dos 76 problemas restantes, em que ambos os métodos encontram uma solução viável e com o mesmo valor de função objetivo, considerando uma tolerância de 10\% no tempo, IALGENCAN é mais rápido em 43 deles, enquanto que o BonMIN é mais rápido em 33 problemas.

Os dados das Tabelas 3.5 e 3.6 indicam que o IALGEncAn é mais rápido do que o Bonmin para esse conjunto de instâncias. Entretanto, IALGENCAN mostra-se inferior no que diz respeito à robustez. De acordo com a Tabela 3.6, nos casos em que ambos encontram uma solução viável, em 13 problemas a solução encontrada pelo BonMIN tem um valor de função melhor do que o da solução encontrada pelo IALGENCAN. Isso indica que IALGENCAN está perdendo a solução global nesses problemas não-convexos e uma das possibilidades para contornar esse problema é alterar o parâmetro da estratégia de reinícios 
e aumentar o número de resoluções de subproblemas em cada nó da árvore. Alternativamente, podemos relaxar o critério de corte dos nós, conforme descrito na Seção 3.2.4, com a esperança de que nós contendo uma solução global para o problema não sejam cortados. 


\section{Capítulo 4}

\section{Detalhes de implementação}

Apresentamos, nesta seção, alguns detalhes relacionados à implementação do algoritmo descrito na Seção 3.2.1, no qual o AlgencAn é usado para resolver as relaxações dos subproblemas do método branch and bound. Na Seção 4.1 abordamos a representação em ponto flutuante das variáveis inteiras, na Seção 4.2 tratamos do arredondamento de soluções como forma de obter soluções viáveis que satisfaçam as restrições de integralidade e na Seção 4.3 exploramos os detalhes da geração de pontos iniciais para a estratégia de reinícios.

\subsection{Número inteiro em ponto flutuante}

No método branch and bound, após a resolução do problema relaxado, precisamos saber se a solução deste problema, caso seja uma solução viável do problema relaxado, também é uma solução viável do problema principal. Caso seja inviável para o problema principal, precisamos descobrir quais variáveis não satisfazem as restrições de integralidade. Ou seja, em última análise, precisamos determinar quando o valor de cada uma das variáveis inteiras pode ser considerado um número inteiro.

Para saber se um determinado número $a$ (diferente de zero) representado em ponto flutuante pode ser considerado um número inteiro, verificamos se

$$
\frac{\min \{a-\lfloor a\rfloor,\lceil a\rceil-a\}}{\max \{1,|a|\}} \leq \epsilon_{\text {mach }},
$$

onde $\epsilon_{\text {mach }}$ (chamado de "epsilon da máquina") é o menor número positivo representável em ponto flutuante tal que $1+\epsilon_{\text {mach }} \neq 1$. Isto é, verificamos se a distância entre $a$ e o número inteiro mais próximo de a é relativamente pequena. Em nosso caso especificamente,

$$
\epsilon_{\text {mach }}=2.220446049250313 \cdot 10^{-16} .
$$

A razão para não se fazer a medição apenas dos erros absolutos $a-\lfloor a\rfloor$ entre $a$ e $\lfloor a\rfloor$ e $\lceil a\rceil-a$ entre $a$ e $\lceil a\rceil$ é que esta é dependente de fatores de escala de $a$, enquanto que a medida dada pelo erro relativo não é. 
Entretanto, tal verificação pode ser muito exigente, de forma que poderíamos considerar como tolerância apenas a raiz quadrada do "epsilon da máquina", e aceitar um número a como sendo um número inteiro se ele satisfizer

$$
\frac{\min \{a-\lfloor a\rfloor,\lceil a\rceil-a\}}{\max \{1,|a|\}} \leq \sqrt{\epsilon_{\text {mach }}}
$$

Esta última garante que aproximadamente metade dos dígitos de $a$ e do inteiro mais próximo de $a$ coincidem.

Em nossa implementação, optamos por usar o critério (4.1).

\subsection{Arredondamento de soluções}

Uma técnica que pode ser utilizada para tentar encontrar uma solução que satisfaça as restrições de integralidade do problema é o arredondamento das soluções provenientes da resolução do problema relaxado. O valor de cada uma das variáveis inteiras é arredondado para o número inteiro mais próximo. No entanto, o arredondamento pode resultar em uma solução inviável do problema original, como mostra o exemplo a seguir. Considere o problema abaixo.

$$
\begin{array}{lr}
\text { Minimizar } & y_{1}\left(1-y_{2}\right)+\ln \left(y_{1}+1\right) \\
\text { sujeita a } & 1.5 y_{1}-y_{2}+1 \geq 0 \\
-1.5 y_{1}-y_{2}+2 & \leq 0 \\
-2 y_{1}-y_{2}+6 & \geq 0 \\
& y \in \mathbb{Z}_{+}^{2}
\end{array}
$$

A solução global deste problema é $y^{*}=(2,2)^{T}$ e tem valor -0.9013 . Partindo do ponto $y=(0,0)^{T}$, a solução do problema relaxado encontrada pelo AlGENCAN é $y_{r}=$ $(1.4285,3.1428)^{T}$. A solução arredondada $y_{a}=(1,3)^{T}$ é inviável, pois não satisfaz a primeira restrição. A Figura 4.1 ilustra esse problema.

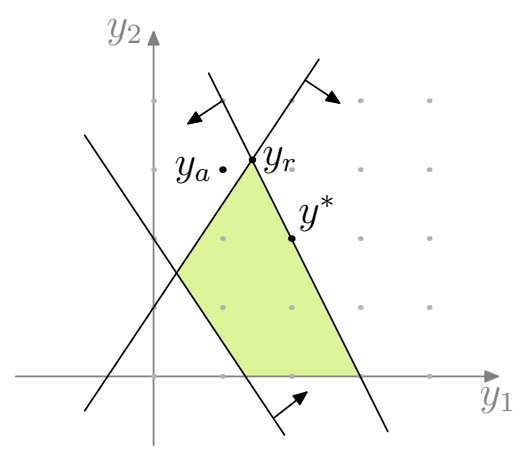

Figura 4.1: Exemplo do procedimento de arredondamento que resulta em um ponto inviável. A solução do problema relaxado é $y_{r}$. Arredondando essa solução, obtemos $y_{a}$, um ponto inviável do problema principal. 
Contudo, o arredondamento de uma solução é um processo relativamente barato, visto que consome tempo linear no número de variáveis e exige a avaliação das restrições para verificar a viabilidade da solução arredondada. Além disso, pode ser muito vantajoso, tendo em vista a possibilidade de obtenção de um novo limitante superior para o problema rapidamente.

Em nossa implementação do método branch and bound, após cada resolução do subproblema relaxado, verificamos se a solução arredondada satisfaz todas as restrições do problema principal. Em caso positivo, atualizamos a melhor solução conhecida para o problema principal se necessário. Independentemente do ponto arredondado satisfazer ou não as restrições do problema principal, o método branch and bound prossegue normalmente conforme descrito no Capítulo 2.

\subsection{Pontos iniciais}

Considere um subproblema no método branch and bound. Sabemos que o que diferencia esse subproblema de qualquer um de seus problemas filhos é apenas uma restrição de igualdade ou desigualdade em uma das variáveis. Isso sugere que a solução da relaxação do subproblema pode estar próxima da solução da relaxação de um de seus problemas filhos. Sendo assim, um bom ponto de partida para um determinado subproblema parece ser o ponto dado pela solução da relaxação do seu problema pai.

Entretanto, o uso da estratégia de reinícios exige diversos pontos iniciais. Podemos, então, tomar como primeiro ponto inicial para um subproblema a solução da relaxação do seu problema pai e obter os demais de alguma outra forma.

Como foi dito, a estratégia de reinícios consiste em resolver um mesmo problema diversas vezes, fornecendo ao algoritmo pontos iniciais distintos. Esses pontos iniciais estão distribuídos sobre toda a região viável do problema e espera-se, com isso, que o algoritmo seja capaz de encontrar soluções viáveis ou até mesmo a solução global do problema.

Precisamos definir uma forma de gerar esses pontos iniciais. Uma possibilidade é gerarmos pontos iniciais de forma pseudo-aleatória. Schrage [67] propôs um gerador de números pseudo-aleatórios que produz uma seqüência de números que é independente de máquina, desde que esta seja capaz de representar números inteiros entre $-2^{31}+1$ e $2^{31}-1$.

No entanto, para que possamos ter um certo controle sobre os experimentos, seria interessante que subproblemas iguais começassem com pontos iniciais iguais, independentemente da localização do subproblema na árvore de busca. Com isso, a estratégia de seleção dos nós (ver Seção 2.3) não iria interferir nos pontos iniciais de determinado subproblema e os experimentos com as diferentes estratégias de seleção de nós seriam comparáveis.

Para isso, precisamos saber o que diferencia um subproblema de outro. Observando o método branch and bound, vemos que a diferença de um (sub)problema para o seu filho (etapa de divisão) é apenas uma restrição em uma das variáveis (justamente na variável de divisão). Essa restrição é uma restrição de limite inferior ou de limite superior, no caso da variável ser do tipo inteira, ou uma restrição que fixa o valor da variável, no caso da variável ser binária ou discreta. Dessa forma, podemos distinguir dois subproblemas 
na árvore apenas olhando para as restrições nas variáveis inteiras. Tais restrições são restrições de caixa, isto é, são da forma $y \in \Omega_{y}$, onde $\Omega_{y}=\left\{y \in \mathbb{R}^{r} \mid l_{y} \leq y \leq u_{y}\right\}$. (No caso da restrição que tem o papel de fixar o valor de uma variável, por exemplo $y_{i}=v$, basta igualar os limitantes inferior e superior desta variável a $v\left(\left[l_{y}\right]_{i}=\left[u_{y}\right]_{i}=v\right)$ que teremos uma restrição de caixa.)

O algoritmo proposto por Schrage foi implementado em Fortran e pode ser encontrado em [67]. A função recebe um parâmetro (semente) que deve ser um inteiro no intervalo $\left(0,2^{31}-1\right)$ e devolve um número inteiro no intervalo $(0,1)$ e um novo valor para a semente que é usado para determinar o próximo número da seqüência.

Sendo a semente responsável por determinar a seqüência de números gerados, precisamos de sementes iguais para problemas iguais. Podemos fazer isso da seguinte forma: calculamos o valor

$$
s=\sum_{i=1}^{r} i\left(\left|\left[l_{y}\right]_{i}\right|+\left|\left[u_{y}\right]_{i}\right|\right)
$$

e tomamos como semente o valor $\lfloor s\rfloor$, desde que $\lfloor s\rfloor \in\left(0,2^{31}-1\right)$, como é exigido pela função. Se $\lfloor s\rfloor \notin\left(0,2^{31}-1\right)$, tomamos como semente algum valor pré-definido. Note que, mesmo tendo a garantia de que subproblemas iguais tenham a mesma semente, não podemos garantir que subproblemas diferentes tenham sementes distintas. O Algoritmo 4.3.1 apresenta a geração dos pontos iniciais.

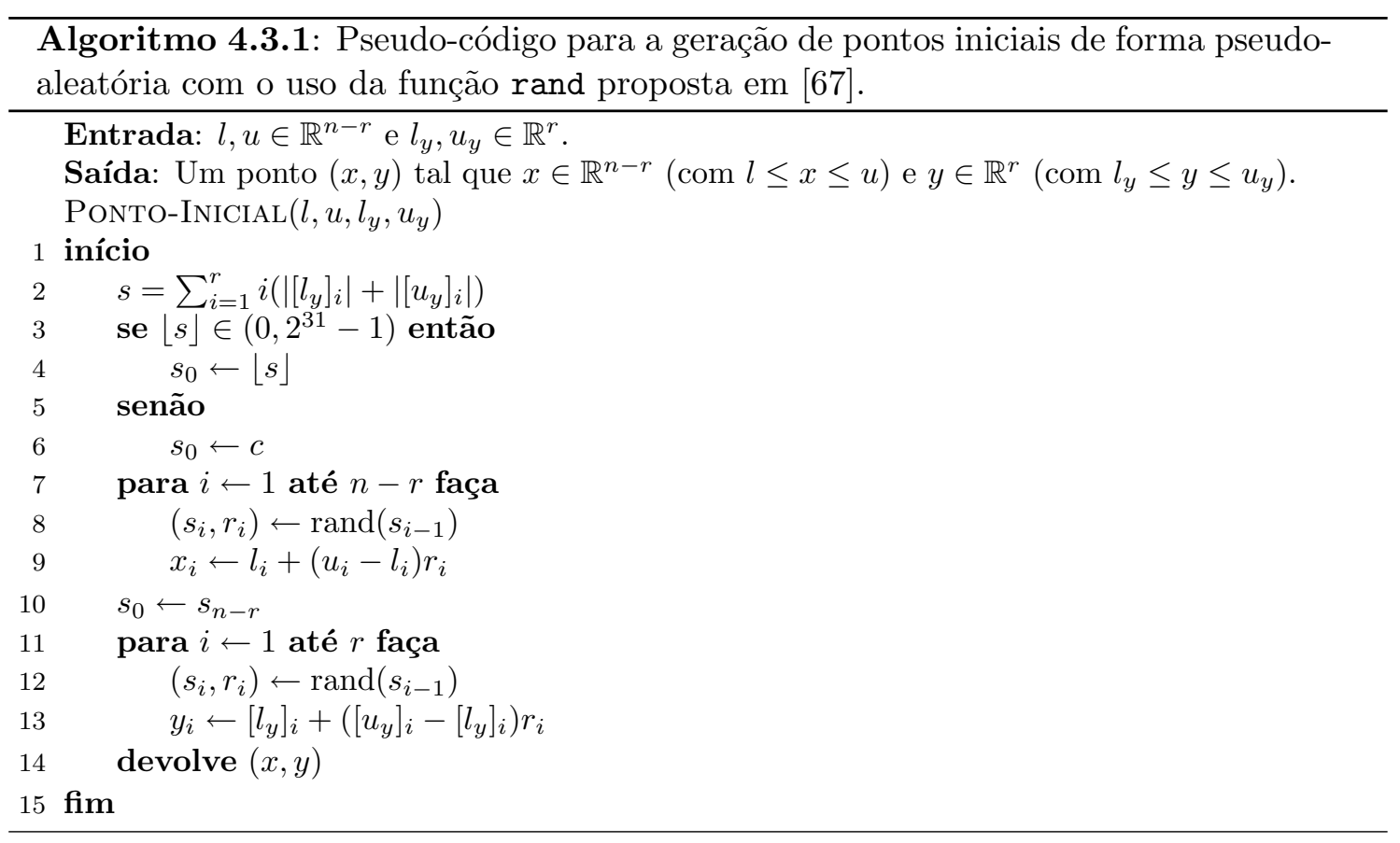




\section{Capítulo 5}

\section{Problemas de empacotamento}

Apresentamos neste capítulo alguns problemas de empacotamento bidimensional de retângulos que podem ser modelados por meio de programação não-linear inteira mista. Na Seção 5.1 introduzimos os dois problemas considerados por Birgin et al. [18]. Nesses problemas o objetivo é empacotar o maior número de retângulos dentro de regiões convexas arbitrárias sem que eles se sobreponham.

Inicialmente, Birgin et al. [18] consideraram o problema de empacotar retângulos em regiões convexas impondo algumas restrições sobre a orientação dos retângulos. Os retângulos deveriam ser posicionados paralelamente aos eixos cartesianos do plano (ou seja, seus lados deveriam permanecer paralelos aos eixos cartesianos) e nenhum tipo de rotação dos retângulos seria permitida, isto é, a orientação de cada um dos retângulos deveria ser fixa. Em seguida, consideraram o problema em que são permitidas rotações ortogonais dos retângulos, ou seja, cada retângulo pode ser rotacionado de um ângulo de 90 graus, mas seus lados ainda devem permanecer paralelos aos eixos cartesianos do plano.

Um problema semelhante foi tratado por Birgin et al. [17]. O problema em questão também tratava do empacotamento de retângulos em regiões convexas, mas permitia que cada um dos retângulos fosse rotacionado de um ângulo arbitrário em relação aos eixos do plano. Nesse problema, o grau de liberdade de cada um dos retângulos é ampliado de tal forma que é possível encontrar soluções contendo um número maior de retângulos do que os presentes nas soluções encontradas nos problemas abordados em [18]. Contudo, o problema também torna-se muito mais difícil de ser resolvido.

Na Seção 5.2, abordamos um problema intermediário aos apresentados em [17] e [18]. Nesse problema, os retângulos devem ser posicionados ortogonalmente entre si, mas eles podem ser rotacionados de um ângulo qualquer em relação aos eixos do plano. Esse problema pode ser considerado intermediário já que os retângulos continuam ortogonais entre si, assim como no problema tratado em [18], e podem ser rotacionados em conjunto de um mesmo ângulo, o que é uma restrição em relação ao problema considerado em [17]. A Figura 5.1 mostra algumas soluções para esses três tipos de problemas. Na Seção 5.3, apresentamos os resultados obtidos por nosso método na resolução do problema descrito na Seção 5.2 e fazemos uma comparação com os resultados alcançados em [17] e [18]. 


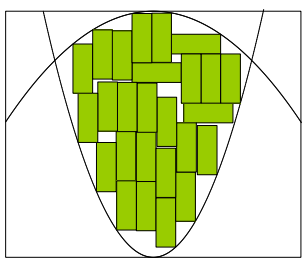

(a)

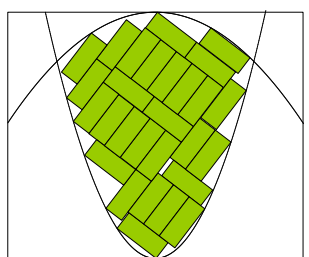

(b)

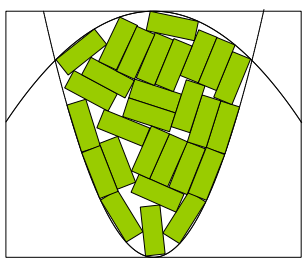

(c)

Figura 5.1: (a) Solução para o problema tratado em [18]. (b) Solução para o problema intermediário introduzido neste capítulo. (c) Solução para o problema abordado em [17].

Notação: Dado um ponto $v \in \mathbb{R}^{2},[v]_{x}$ denota a coordenada horizontal de $v$ e $[v]_{y}$ denota a coordenada vertical de $v$.

\subsection{Empacotamento ortogonal de retângulos}

Birgin et al. [18] consideraram o empacotamento de retângulos em regiões convexas arbitrárias. Um primeiro problema consiste em empacotar um conjunto de $m$ retângulos em uma dada região convexa $\Omega \subset \mathbb{R}^{2}$. Cada retângulo deve estar inteiramente dentro da região especificada e não pode existir sobreposição entre os retângulos. Além disso, nenhum tipo de rotação dos retângulos é permitida, de modo que um dos lados do retângulo estará sempre paralelo a um determinado eixo (vertical ou horizontal).

Para todo $i=1, \ldots, m$, seja $C^{i}=\left(c_{1}^{i}, c_{2}^{i}\right)$ a variável que indica o centro do retângulo $R^{i} \equiv R^{i}\left(a^{i}, b^{i}\right)$, onde $a^{i}, b^{i}>0$ são respectivamente os tamanhos dos lados horizontal (que será paralelo ao eixo horizontal) e vertical (que será paralelo ao eixo vertical) do retângulo $R^{i}$. Sejam

$$
\begin{aligned}
V_{\mathrm{SW}}^{i} \equiv V_{\mathrm{sw}}^{i}\left(R^{i}, C^{i}\right) & =\left(c_{1}^{i}-a^{i} / 2, c_{2}^{i}-b^{i} / 2\right), \\
V_{\mathrm{se}}^{i} \equiv V_{\mathrm{se}}^{i}\left(R^{i}, C^{i}\right) & =\left(c_{1}^{i}+a^{i} / 2, c_{2}^{i}-b^{i} / 2\right), \\
V_{\mathrm{ne}}^{i} \equiv V_{\mathrm{ne}}^{i}\left(R^{i}, C^{i}\right) & =\left(c_{1}^{i}+a^{i} / 2, c_{2}^{i}+b^{i} / 2\right) \mathrm{e} \\
V_{\mathrm{nw}}^{i} \equiv V_{\mathrm{nw}}^{i}\left(R^{i}, C^{i}\right) & =\left(c_{1}^{i}-a^{i} / 2, c_{2}^{i}+b^{i} / 2\right)
\end{aligned}
$$

os vértices do retângulo $R^{i}$ com centro em $C^{i}$ e seja $D=\{\mathrm{sw}$, se, ne, nw $\}$ o conjunto dos identificadores dos vértices do retângulo, como ilustra a Figura 5.2. Este problema pode

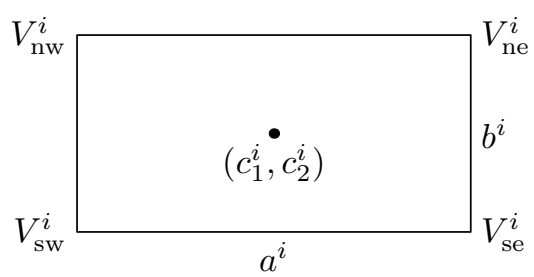

Figura 5.2: Vértices e centro do retângulo $i$ com dimensões $a^{i}$ e $b^{i}$.

ser enunciado da seguinte forma: 
Problema FOP: Dados um subconjunto convexo $\Omega$ de $\mathbb{R}^{2}$ e um conjunto de $m$ retângulos $R^{i} \equiv R^{i}\left(a^{i}, b^{i}\right), i=1, \ldots, m$, encontrar $C^{1}, \ldots, C^{m}$ tais que:

1. Para todo $i=1, \ldots, m$ e $d \in D$,

$$
V_{d}^{i} \in \Omega
$$

2. Para todo $i, j=1, \ldots, m, i \neq j$,

$$
\left|c_{1}^{i}-c_{1}^{j}\right| \geq \frac{a^{i}+a^{j}}{2}
$$

$\mathrm{Ou}$

$$
\left|c_{2}^{i}-c_{2}^{j}\right| \geq \frac{b^{i}+b^{j}}{2}
$$

A condição (5.2) diz que os vértices de cada retângulo $R^{i}$ devem estar dentro da região convexa $\Omega$. Note que essa condição é necessária e suficiente para garantir que os retângulos estejam inteiramente dentro da região desejada, já que $\Omega$ é uma região convexa. O fato de pelo menos uma das condições (5.3) ou (5.4) ser satisfeita significa que os retângulos $R^{i}$ e $R^{j}$ não estão sobrepostos. O problema FOP é um problema de viabilidade, mas ele também pode ser visto como o problema de encontrar uma solução global de

$$
\begin{array}{ll}
\underset{\text { minimizar }}{\operatorname{mita}} & \bar{f}\left(C^{1}, \ldots, C^{m}\right) \\
\text { sujeita } & (5.2)
\end{array}
$$

onde

$$
\begin{aligned}
\bar{f}\left(C^{1}, \ldots, C^{m}\right)=\sum_{i=1}^{m-1} \sum_{j=i+1}^{m} & {\left[\max \left\{0, \frac{\left(a^{i}+a^{j}\right)^{2}}{4}-\left(c_{1}^{i}-c_{1}^{j}\right)^{2}\right\}^{2}\right.} \\
\times & \left.\max \left\{0, \frac{\left(b^{i}+b^{j}\right)^{2}}{4}-\left(c_{2}^{i}-c_{2}^{j}\right)^{2}\right\}^{2}\right] .
\end{aligned}
$$

A função objetivo do problema (5.5) tem por fim penalizar os pontos que não satisfazem nem a restrição (5.3) e nem restrição (5.4), ou seja, penalizar os pontos que produzem soluções nas quais há sobreposições entre os retângulos. Observe, com isso, que uma solução viável para o problema (5.5) pode não ser uma solução viável para o problema FOP. Mais ainda, note que uma solução em que $c_{1}^{i}=c_{1}^{j}$ e $c_{2}^{i}=c_{2}^{j}$ para todo $i, j$ (inviável para o problema FOP) é um ponto estacionário da função objetivo do problema (5.5). As soluções viáveis de (5.5) são caracterizadas por pontos que produzem retângulos dentro da região viável $\Omega$, mas que possivelmente estejam sobrepostos.

As soluções viáveis de (5.5) que produzem soluções com retângulos não sobrepostos satisfazem (5.3) ou (5.4) e, portanto,

$$
\max \left\{0, \frac{\left(a^{i}+a^{j}\right)^{2}}{4}-\left(c_{1}^{i}-c_{1}^{j}\right)^{2}\right\}^{2} \times \max \left\{0, \frac{\left(b^{i}+b^{j}\right)^{2}}{4}-\left(c_{2}^{i}-c_{2}^{j}\right)^{2}\right\}^{2}=0
$$

para todo $i, j$ com $i \neq j$. Assim, tais soluções anulam o valor da função objetivo e, como $\bar{f}\left(C^{1}, \ldots, C^{m}\right)$ é uma função não negativa, elas são soluções globais de (5.5). É fácil ver 
que uma solução é viável para o problema FOP se, e somente se, ela é uma solução global para o problema (5.5).

Birgin et al. [18] consideraram o caso em que a região convexa $\Omega$ tem a forma $\Omega=$ $\Omega_{1} \cap \Omega_{2}$, onde $\Omega_{1}$ representa as restrições de caixa e $\Omega_{2}$ as demais restrições:

$$
\begin{aligned}
& \Omega_{1}=\left\{x \in \mathbb{R}^{2} \mid l \leq x \leq u\right\} \text { e } \\
& \Omega_{2}=\left\{x \in \mathbb{R}^{2} \mid g_{k}(x) \leq 0 \text { para } k=1, \ldots, r\right\} .
\end{aligned}
$$

Assim, a restrição (5.2) pode ser reescrita como

$$
V_{d}^{i} \in \Omega_{1}
$$

e

$$
V_{d}^{i} \in \Omega_{2}
$$

para $i=1, \ldots, m$ e $d \in D$. Pelo fato de que os pontos que satisfazem (5.7) anulam

$$
\sum_{k=1}^{r} \sum_{i=1}^{m} \sum_{d \in D} \max \left\{0, g_{k}\left(V_{d}^{i}\right)\right\}
$$

e vice-versa, o problema (5.5) pode ser reformulado como

$$
\begin{array}{ll}
\underset{\text { minimizar }}{\operatorname{majeita~a}} & \bar{f}\left(C^{1}, \ldots, C^{m}\right)+\sum_{k=1}^{r} \sum_{i=1}^{m} \sum_{d \in D} \max \left\{0, g_{k}\left(V_{d}^{i}\right)\right\} \\
\text { suje } &
\end{array}
$$

O problema (5.8) é um problema de minimização em caixas e Birgin et al. [18] decidiram resolvê-lo usando o GENCAN [15], um método específico para esse tipo de problema.

A fim de permitir rotações de 90 graus, comum em muitas aplicações, podemos escolher para cada retângulo $R^{i}, i=1, \ldots, m$, se seu lado horizontal será o de tamanho $a^{i}$ ou o de tamanho $b^{i}$. Se o problema FOP for resolvido com esta escolha, então o problema de empacotamento com rotações ortogonais também estará resolvido. Apesar de existirem $2^{m}$ possibilidades de configurações dos retângulos (cada retângulo pode ter o seu lado de comprimento $a^{i}$ paralelo ao eixo horizontal ou ao eixo vertical), este número pode ser reduzido em determinadas situações. Por exemplo, no caso em que $a^{i}=a$ e $b^{i}=b$, para $i=1, \ldots, m$, o número de configurações possíveis pode ser reduzido para $m+1$. Isso porque, como os retângulos são todos idênticos, só nos interessa saber o número $p$ de retângulos com o lado de comprimento $a$ paralelo ao eixo horizontal. Como $p$ pode ser 0 , $1, \ldots, m-1$ ou $m$, temos um total de $m+1$ configurações possíveis.

Assim, o problema de empacotar ortogonalmente $m$ retângulos idênticos em uma região convexa pode ser definido como segue.

Problema ORP: Dados um conjunto convexo $\Omega \subset \mathbb{R}^{2}$ e um conjunto de $m$ retângulos idênticos $R^{i} \equiv R^{i}(a, b)$, encontrar $C^{1}, \ldots, C^{m} \in \mathbb{R}^{2}$ e $p \in\{0, \ldots, m\}$ tais que $\left(C^{1}, \ldots, C^{m}\right)$ é uma solução do problema FOP definido pelos retângulos $\bar{R}^{i} \equiv \bar{R}^{i}(a, b), i=1, \ldots, p$, 


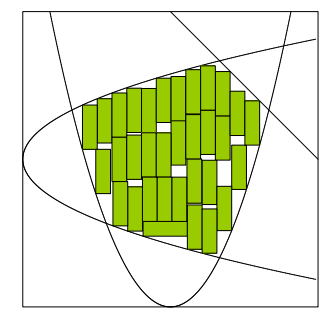

Figura 5.3: Representação gráfica de uma solução para o problema ORP em que $m=33$, $a=0.9, b=0.3$ e $\Omega=\left\{x \in \mathbb{R}^{2} \mid x_{1}^{2}-x_{2} \leq 0,-x_{1}+x_{2}^{2}-6 x_{2}+6 \leq 0, x_{1}+x_{2}-6 \leq 0\right\}$.

$\bar{R}^{i} \equiv \bar{R}^{i}(b, a), i=p+1, \ldots, m$ e pelo conjunto $\Omega$.

A versão de otimização do problema ORP é um exemplo de problema de otimização não-linear com variáveis inteiras e contínuas. Ela apresenta não-linearidades na função objetivo e possui variáveis contínuas $\left(C^{1}, \ldots, C^{m}\right)$ e uma variável inteira $(p)$. A Figura 5.3 ilustra uma solução deste problema para o caso em que $m=33, a=0.9, b=0.3$ e

$$
\Omega=\left\{x \in \mathbb{R}^{2} \mid x_{1}^{2}-x_{2} \leq 0,-x_{1}+x_{2}^{2}-6 x_{2}+6 \leq 0, x_{1}+x_{2}-6 \leq 0\right\} .
$$

\subsection{Empacotamento ortogonal com rotação}

O problema ORP descrito na Seção 5.1 exige que os retângulos tenham seus lados paralelos aos eixos cartesianos. Uma variação desse problema permite que os retângulos sejam rotacionadas de um ângulo qualquer desde que mantenham ortogonalidade entre si, isto é, cada retângulo deve ter um de seus lados paralelo a um dos lados de cada um dos outros retângulos. Chamaremos esse problema de "problema de empacotamento ortogonal com rotação" (EOR). A Figura 5.4 mostra uma solução para um problema desse tipo.

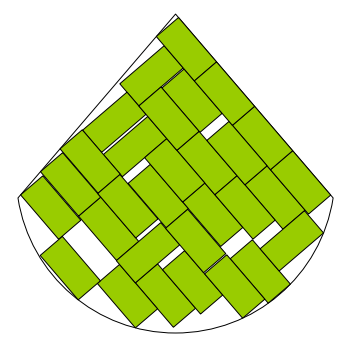

Figura 5.4: Exemplo de solução para o problema de empacotamento ortogonal com rotação. Note que os retângulos estão rotacionados em relação ao eixo cartesiano, mas estão posicionados ortogonalmente entre si.

Seja $D$ o conjunto de identificadores dos vértices dos retângulos como definido anteriormente e sejam $V_{d}^{i, \theta}$, para $d \in D$, os vértices do retângulo $i$. Formalmente, podemos definir esse problema da seguinte forma. 
Problema EOR: Dados um conjunto convexo $\Omega \subset \mathbb{R}^{2}$ e um conjunto de $m$ retângulos idênticos $R^{i} \equiv R^{i}(a, b)$, encontrar $C^{1}, \ldots, C^{m} \in \mathbb{R}^{2}, \theta \in[0,2 \pi]$ e $p \in\{0, \ldots, m\}$ tais que:

1. Para todo $i=1, \ldots, m$ e $d \in D$,

$$
V_{d}^{i, \theta} \in \Omega
$$

2. Para todo $i, j=1, \ldots, m, i \neq j$,

o retângulo $i$ não deve se sobrepor ao retângulo $j$.

A seguir, nas Seções 5.2.1 e 5.2.2, apresentamos dois modelos para esse problema. Na Seção 5.2.1, apresentamos um modelo cuja função objetivo tem o inconveniente de ser não diferenciável. Já na Seção 5.2.2, contornamos o problema da não diferenciabilidade propondo um outro modelo.

\subsubsection{Primeiro modelo}

O problema EOR pode ser modelado da seguinte forma. Assim como no problema ORP, seja $C^{i}=\left(c_{1}^{i}, c_{2}^{i}\right) \in \mathbb{R}^{2}$ a variável que indica o centro do retângulo $i$, para $i=$ $1, \ldots, m$. Seja $\theta \in[0,2 \pi]$ o ângulo de rotação dos retângulos relativamente aos eixos cartesianos. Seja $p \in\{0, \ldots, m\}$ a variável inteira que indica o número de retângulos que possuem o lado de comprimento a paralelo ao eixo horizontal rotacionado de $\theta$. Assim como no problema ORP e sem perda de generalidade, teremos $R^{i} \equiv R^{i}\left(a^{i}, b^{i}\right)=R^{i}(a, b)$ para $i=1, \ldots, p$ e $R^{i} \equiv R^{i}\left(a^{i}, b^{i}\right)=R^{i}(b, a)$ para $i=p+1, \ldots, m$.

Agora, precisamos definir um modo de detectar a sobreposição entre retângulos. Como vimos, no caso do problema FOP, a condição para que não haja sobreposição entre os retângulos é

$$
\left|c_{1}^{i}-c_{1}^{j}\right| \geq \frac{a^{i}+a^{j}}{2} \quad \text { ou } \quad\left|c_{2}^{i}-c_{2}^{j}\right| \geq \frac{b^{i}+b^{j}}{2}
$$

para todo $i, j=1, \ldots, m$ com $i \neq j$. Contudo, essa condição só é válida para o caso em que os retângulos têm seus lados paralelos aos eixos cartesianos. No problema EOR, os retângulos podem estar rotacionados em relação aos eixos cartesianos e nesse caso (5.11) não é mais uma condição para evitar sobreposições.

No entanto, a condição (5.11) ainda pode ser aproveitada para o problema EOR, bastando uma pequena modificação. Uma possibilidade para detectar as sobreposições entre os retângulos rotacionados é "desfazer" as rotações (isto é, rotacioná-los de um ângulo $-\theta)$. Sendo $\hat{C}^{i}=\left(\hat{c}_{1}^{i}, \hat{c}_{2}^{i}\right)$ o centro do retângulo $i$ após rotacioná-lo de $-\theta$, podemos usar a condição (5.11) com $\left(\hat{c}_{1}^{i}, \hat{c}_{2}^{i}\right)$ no lugar de $\left(c_{1}^{i}, c_{2}^{i}\right)$, e $\left(\hat{c}_{1}^{j}, \hat{c}_{2}^{j}\right)$ no lugar de $\left(c_{1}^{j}, c_{2}^{j}\right)$.

Seja $T(\theta)$ a matriz de rotação dada por

$$
T(\theta)=\left(\begin{array}{rr}
\cos (\theta) & -\sin (\theta) \\
\sin (\theta) & \cos (\theta)
\end{array}\right) .
$$

O centro "desrotacionado" $\hat{C}^{i}$ do retângulo $i$ é então dado por

$$
\hat{C}^{i}=T(-\theta) C^{i}=\left(\begin{array}{c}
c_{1}^{i} \cos (-\theta)-c_{2}^{i} \sin (-\theta) \\
c_{1}^{i} \sin (-\theta)+c_{2}^{i} \cos (-\theta)
\end{array}\right) .
$$




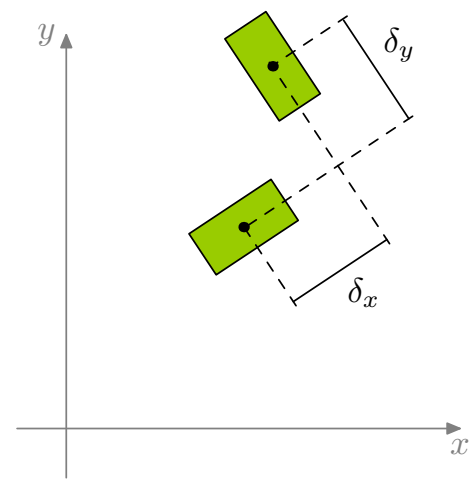

(a)

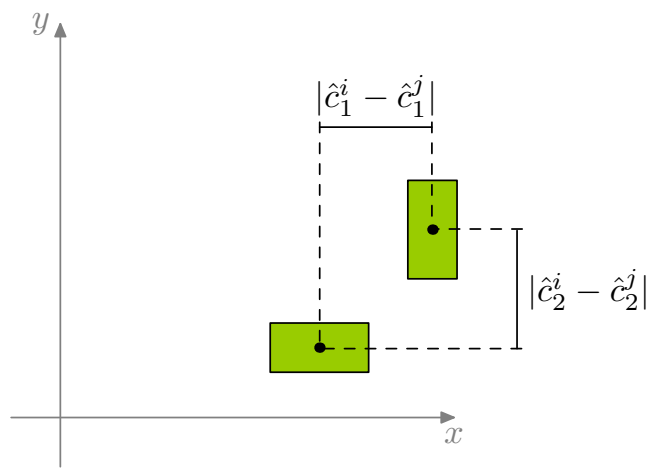

(b)

Figura 5.5: Método para determinar se dois retângulos estão sobrepostos. (a) Posicionamento dos retângulos. (b) Os retângulos rotacionados de $-\theta$ e a fácil verificação de sobreposição.

Para cada retângulo $i$, se $i \in\{1, \ldots, p\}$ (isto é, se o retângulo $i$ tem seu lado de comprimento $a$ paralelo ao eixo horizontal rotacionado), precisamos tomar $\left(a^{i}, b^{i}\right)=(a, b)$, enquanto que se $i>p$ devemos tomar $\left(a^{i}, b^{i}\right)=(b, a)$. Para isso podemos construir uma função indicadora $I(i, p)$ que diz se $i$ está em $\{1, \ldots, p\}$. Por exemplo, podemos tomar

$$
I(i, p)= \begin{cases}1 & i \leq p \\ 0 & i>p\end{cases}
$$

desde que $1 \leq i \leq m$. Definindo

$$
I(i, p)=\min (\max (0, p-i), 1)
$$

obtemos a função desejada. Sendo assim, os lados $\left(a_{p}^{i}, b_{p}^{i}\right)$ do retângulo $i$ são dados por

$$
\begin{aligned}
a_{p}^{i} & =I(i, p) a+(1-I(i, p)) b \\
b_{p}^{i} & =I(i, p) b+(1-I(i, p)) a .
\end{aligned}
$$

Então, a condição para que não haja sobreposição entre os retângulos $i$ e $j$ passa a ser

$$
\left|\hat{c}_{1}^{i}-\hat{c}_{1}^{j}\right| \geq \frac{a_{p}^{i}+a_{p}^{j}}{2} \quad \text { ou } \quad\left|\hat{c}_{2}^{i}-\hat{c}_{2}^{j}\right| \geq \frac{b_{p}^{i}+b_{p}^{j}}{2} .
$$

A Figura 5.5 ilustra a condição de não sobreposição dos retângulos.

Agora nos resta saber como verificar a condição (5.9), que diz que os vértices dos retângulos devem permanecer dentro da região $\Omega$. No problema FOP, dado o centro de um retângulo, seus vértices são facilmente calculados, já que os retângulos têm seus lados paralelos aos eixos cartesianos. Assim, basta adicionar ou subtrair apropriadamente ao centro do retângulo a metade dos tamanhos de seus lados para se obter os vértices do 


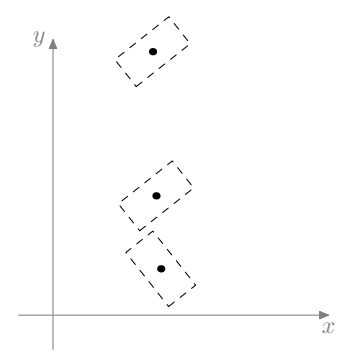

(a)

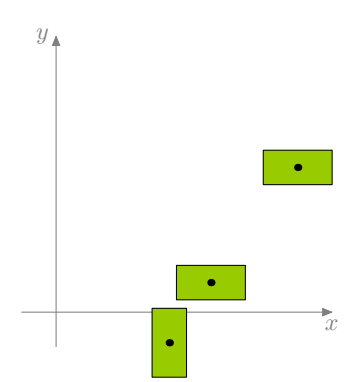

(b)

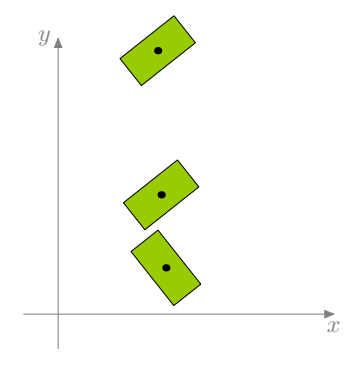

(c)

Figura 5.6: Ilustração do procedimento de determinação dos vértices dos retângulos. (a) Os centros dos retângulos com seus vértices ainda desconhecidos. (b) "Desrotação" dos retângulos seguida do cálculo trivial dos vértices $\hat{V}_{d}^{i, \theta}$. (c) Rotação dos vértices dos retângulos e fim do procedimento.

retângulo. No caso do problema EOR essa tarefa pode ser mais complicada, já que os retângulos estão rotacionadas de um ângulo qualquer.

Um possibilidade para calcular os vértices de um retângulo é utilizar a mesma estratégia usada para a detecção de sobreposições, por meio da "desrotação" dos retângulos. Assim, os vértices do retângulo $i$ podem ser determinados através do seguinte procedimento:

1. rotacionar o retângulo $i$ de um ângulo $-\theta$, obtendo centro $\hat{C}^{i}=T(-\theta) C^{i}$;

2. calcular os vértices do retângulo (analogamente a (5.1)), obtendo vértices $\hat{V}_{d}^{i, \theta}$ para $d \in D$ :

$$
\begin{aligned}
\hat{V}_{\mathrm{sW}}^{i, \theta} \equiv \hat{V}_{\mathrm{sw}}^{i}\left(R^{i}, C^{i}, \theta, p\right) & =\left(\hat{c}_{1}^{i}-a_{p}^{i} / 2, \hat{c}_{2}^{i}-b_{p}^{i} / 2\right), \\
\hat{V}_{\mathrm{se}}^{i, \theta} \equiv \hat{V}_{\mathrm{se}}^{i}\left(R^{i}, C^{i}, \theta, p\right) & =\left(\hat{c}_{1}^{i}+a_{p}^{i} / 2, \hat{c}_{2}^{i}-b_{p}^{i} / 2\right), \\
\hat{V}_{\mathrm{ne}}^{i, \theta} \equiv \hat{V}_{\mathrm{ne}}^{i}\left(R^{i}, C^{i}, \theta, p\right) & =\left(\hat{c}_{1}^{i}+a_{p}^{i} / 2, \hat{c}_{2}^{i}+b_{p}^{i} / 2\right) \mathrm{e} \\
\hat{V}_{\mathrm{nw}}^{i, \theta} \equiv \hat{V}_{\mathrm{nW}}^{i}\left(R^{i}, C^{i}, \theta, p\right) & =\left(\hat{c}_{1}^{i}-a_{p}^{i} / 2, \hat{c}_{2}^{i}+b_{p}^{i} / 2\right) ;
\end{aligned}
$$

3. rotacionar de um ângulo $\theta$ os vértices calculados, obtendo vértices $V_{d}^{i, \theta}=T(\theta) \hat{V}_{d}^{i, \theta}$, para $d \in D$.

A Figura 5.6 ilustra esse procedimento. Explicitamente, os vértices $V_{\mathrm{sw}}^{i, \theta}, V_{\mathrm{se}}^{i, \theta}, V_{\mathrm{ne}}^{i, \theta}$ e $V_{\mathrm{nw}}^{i, \theta}$ do retângulo $i$ são dados por:

$$
\begin{aligned}
{\left[V_{\mathrm{Sw}}^{i, \theta}\right]_{x}=} & {\left[\left(c_{1}^{i} \cos (-\theta)-c_{2}^{i} \sin (-\theta)\right)-(I(i, p) a+(1-I(i, p)) b) / 2\right] \cos (\theta)-} \\
& {\left[\left(c_{1}^{i} \sin (-\theta)+c_{2}^{i} \cos (-\theta)\right)-(I(i, p) b+(1-I(i, p)) a) / 2\right] \sin (\theta) } \\
{\left[V_{\mathrm{Sw}}^{i, \theta}\right]_{y}=} & {\left[\left(c_{1}^{i} \cos (-\theta)-c_{2}^{i} \sin (-\theta)\right)-(I(i, p) a+(1-I(i, p)) b) / 2\right] \sin (\theta)+} \\
& {\left[\left(c_{1}^{i} \sin (-\theta)+c_{2}^{i} \cos (-\theta)\right)-(I(i, p) b+(1-I(i, p)) a) / 2\right] \cos (\theta) }
\end{aligned}
$$




$$
\begin{aligned}
& {\left[V_{\mathrm{Se}}^{i, \theta}\right]_{x}=} {\left[\left(c_{1}^{i} \cos (-\theta)-c_{2}^{i} \sin (-\theta)\right)+(I(i, p) a+(1-I(i, p)) b) / 2\right] \cos (\theta)-} \\
& {\left[\left(c_{1}^{i} \sin (-\theta)+c_{2}^{i} \cos (-\theta)\right)-(I(i, p) b+(1-I(i, p)) a) / 2\right] \sin (\theta) } \\
& {\left[V_{\mathrm{se}}^{i, \theta}\right]_{y}=} {\left[\left(c_{1}^{i} \cos (-\theta)-c_{2}^{i} \sin (-\theta)\right)+(I(i, p) a+(1-I(i, p)) b) / 2\right] \sin (\theta)+} \\
& {\left[\left(c_{1}^{i} \sin (-\theta)+c_{2}^{i} \cos (-\theta)\right)-(I(i, p) b+(1-I(i, p)) a) / 2\right] \cos (\theta) } \\
& {\left[V_{\mathrm{ne}}^{i, \theta}\right]_{x}=} {\left[\left(c_{1}^{i} \cos (-\theta)-c_{2}^{i} \sin (-\theta)\right)+(I(i, p) a+(1-I(i, p)) b) / 2\right] \cos (\theta)-} \\
& {\left[\left(c_{1}^{i} \sin (-\theta)+c_{2}^{i} \cos (-\theta)\right)+(I(i, p) b+(1-I(i, p)) a) / 2\right] \sin (\theta) } \\
& {\left[V_{\mathrm{ne}}^{i, \theta}\right]_{y}=} {\left[\left(c_{1}^{i} \cos (-\theta)-c_{2}^{i} \sin (-\theta)\right)+(I(i, p) a+(1-I(i, p)) b) / 2\right] \sin (\theta)+} \\
& {\left[\left(c_{1}^{i} \sin (-\theta)+c_{2}^{i} \cos (-\theta)\right)+(I(i, p) b+(1-I(i, p)) a) / 2\right] \cos (\theta) } \\
& {\left[\left(c_{1}^{i} \cos (-\theta)-c_{2}^{i} \sin (-\theta)\right)-(I(i, p) a+(1-I(i, p)) b) / 2\right] \cos (\theta)-} \\
& {\left[\left(c_{1}^{i} \sin (-\theta)+c_{2}^{i} \cos (-\theta)\right)+(I(i, p) b+(1-I(i, p)) a) / 2\right] \sin (\theta) } \\
& {\left[V_{\mathrm{nw}}^{i, \theta}\right]_{x}=} {\left[\left(c_{1}^{i} \cos (-\theta)-c_{2}^{i} \sin (-\theta)\right)-(I(i, p) a+(1-I(i, p)) b) / 2\right] \sin (\theta)+} \\
& {\left.\left.\left[V_{\mathrm{nw}}^{i, \theta}\right]_{y}=c_{2}^{i} \cos (-\theta)\right)+(I(i, p) b+(1-I(i, p)) a) / 2\right] \cos (\theta) . }
\end{aligned}
$$

Como $\cos (-\theta)=\cos (\theta)$ e $\sin (-\theta)=-\sin (\theta)$, temos

$$
\begin{aligned}
{\left[V_{\mathrm{sW}}^{i, \theta}\right]_{x}=} & {\left[\left(c_{1}^{i} \cos (-\theta)-c_{2}^{i} \sin (-\theta)\right)-(I(i, p) a+(1-I(i, p)) b) / 2\right] \cos (\theta)-} \\
& {\left[\left(c_{1}^{i} \sin (-\theta)+c_{2}^{i} \cos (-\theta)\right)-(I(i, p) b+(1-I(i, p)) a) / 2\right] \sin (\theta) } \\
= & {\left[\left(c_{1}^{i} \cos (\theta)+c_{2}^{i} \sin (\theta)\right)-(I(i, p) a+(1-I(i, p)) b) / 2\right] \cos (\theta)-} \\
& {\left[\left(-c_{1}^{i} \sin (\theta)+c_{2}^{i} \cos (\theta)\right)-(I(i, p) b+(1-I(i, p)) a) / 2\right] \sin (\theta) } \\
= & {\left[\left(c_{1}^{i} \cos ^{2}(\theta)+c_{2}^{i} \sin (\theta) \cos (\theta)\right)-\cos (\theta)(I(i, p) a+(1-I(i, p)) b) / 2\right]-} \\
& {\left[\left(-c_{1}^{i} \sin ^{2}(\theta)+c_{2}^{i} \sin (\theta) \cos (\theta)\right)-\sin (\theta)(I(i, p) b+(1-I(i, p)) a) / 2\right] . }
\end{aligned}
$$


Usando a relação $\cos ^{2}(\theta)+\sin ^{2}(\theta)=1$, temos

$$
\left[V_{\mathrm{sw}}^{i, \theta}\right]_{x}=-\cos (\theta)(I(i, p) a+(1-I(i, p)) b) / 2+\sin (\theta)(I(i, p) b+(1-I(i, p)) a) / 2+c_{1}^{i} .
$$

Analogamente, para a componente $\left[V_{\mathrm{Sw}}^{i, \theta}\right]_{y}$ do vértice $V_{\mathrm{Sw}}^{i, \theta}$, temos

$$
\left[V_{\mathrm{sw}}^{i, \theta}\right]_{y}=-\sin (\theta)(I(i, p) a+(1-I(i, p)) b) / 2-\cos (\theta)(I(i, p) b+(1-I(i, p)) a) / 2+c_{2}^{i}
$$

Note que

$$
V_{\mathrm{sw}}^{i, \theta}=T(\theta)\left(\begin{array}{l}
-a_{p}^{i} / 2 \\
-b_{p}^{i} / 2
\end{array}\right)+C^{i}
$$

A expressão (5.13) pode ser interpretada da seguinte forma. O centro do retângulo $i$ é trazido para a origem do sistema cartesiano e, com os lados do retângulo paralelos aos eixos cartesianos, o vértice sw é calculado. Em seguida, o vértice sw é rotacionado de um ângulo $\theta$ e ao ponto resultante é adicionada a coordenada do centro do retângulo $i$. $\mathrm{O}$ ponto produzido é o vértice $V_{\mathrm{SW}}^{i, \theta}$ do retângulo $i$.

Portanto, para o cálculo dos vértices dos retângulos, podemos usar tanto o procedimento que usa "desrotação" quanto este último apresentado. Como este último possui uma expressão visivelmente mais simples, optamos por usá-lo ao invés do primeiro.

Formulando o problema EOR como um problema de otimização, temos:

$$
\begin{array}{ll}
\operatorname{minimizar} & f_{1}(C, p, \theta) \\
\text { sujeita a } & V_{d}^{i, \theta} \in \Omega_{1}, i=1, \ldots, m \text { e } d \in D \\
& p \in\{1, \ldots, m\}
\end{array}
$$

onde

$$
\begin{aligned}
f_{1}(C, p, \theta)= & \sum_{i=1}^{m-1} \sum_{j=i+1}^{m} \max \left\{0,\left(a_{p}^{i}+a_{p}^{j}\right)^{2} / 4-\left(\hat{c}_{1}^{i}-\hat{c}_{1}^{j}\right)^{2}\right\}^{2} \\
& \times \max \left\{0,\left(b_{p}^{i}+b_{p}^{j}\right)^{2} / 4-\left(\hat{c}_{2}^{i}-\hat{c}_{2}^{j}\right)^{2}\right\}^{2} \\
& +\sum_{k=1}^{r} \sum_{i=1}^{m} \sum_{d \in D} \max \left\{0, g_{k}\left(V_{d}^{i, \theta}\right)\right\}^{2} .
\end{aligned}
$$

Substituindo $a_{p}^{i}, a_{p}^{j}, b_{p}^{i}, b_{p}^{j}, \hat{c}_{1}^{i}, \hat{c}_{1}^{j}, \hat{c}_{2}^{i}$ e $\hat{c}_{2}^{j}$ por seus respectivos valores, temos: 


$$
\begin{aligned}
& f_{1}(C, p, \theta)=\sum_{i=1}^{m-1} \sum_{j=i+1}^{m} \max \{0, {[(I(i, p) a+(1-I(i, p)) b)+(I(j, p) a+(1-I(j, p)) b)]^{2} / 4-} \\
& {\left.\left[\left(c_{1}^{i} \cos (-\theta)-c_{2}^{i} \sin (-\theta)\right)-\left(c_{1}^{j} \cos (-\theta)-c_{2}^{j} \sin (-\theta)\right)\right]^{2}\right\}^{2} } \\
& \times \max \left\{0, \quad[(I(i, p) b+(1-I(i, p)) a)+(I(j, p) b+(1-I(j, p)) a)]^{2} / 4-\right. \\
&+\sum_{k=1}^{r} \sum_{i=1}^{m} \sum_{d \in D} \max \{0,\left.g_{k}\left(V_{d}^{i, \theta}\right)\right\}^{2} .
\end{aligned}
$$

Um grande problema que esse modelo apresenta é a não diferenciabilidade da função objetivo $f_{1}$. A função indicadora $I$ que tem o papel de decidir o comprimento dos lados dos retângulos não é diferenciável, o que compromete a resolução do problema, visto que o nosso método requer que a função seja diferenciável.

\subsubsection{Um modelo mais adequado}

O modelo apresentado a seguir possui função objetivo diferenciável, de forma que o nosso método pode ser usado para resolver o problema de empacotamento.

No modelo descrito na Seção 5.2.1, existe uma única variável inteira para determinar o lado de cada um dos retângulos que será paralelo ao eixo horizontal rotacionado, ou seja, para determinar se $\left(a^{i}, b^{i}\right)=(a, b)$ ou se $\left(a^{i}, b^{i}\right)=(b, a)$ para cada $i=1, \ldots, m$. Agora, para cada retângulo $i$, teremos uma variável binária denominada $p^{i}$ que indicará o lado do retângulo $i$ que será paralelo ao eixo horizontal rotacionado. Se $p^{i}=1$ então teremos $\left(a^{i}, b^{i}\right)=(a, b)$ e se $p^{i}=0$, teremos $\left(a^{i}, b^{i}\right)=(b, a)$. Assim, os lados $\left(a_{p^{i}}^{i}, b_{p^{i}}^{i}\right)$ do retângulo $i$ são dados por

$$
\begin{aligned}
& a_{p^{i}}^{i}=p^{i} a+\left(1-p^{i}\right) b \\
& b_{p^{i}}^{i}=p^{i} b+\left(1-p^{i}\right) a .
\end{aligned}
$$

Seja $\hat{C}^{i}=\left(\hat{c}_{1}^{i}, \hat{c}_{2}^{i}\right)$ o centro do retângulo $i$ rotacionado de $-\theta$. De forma análoga ao realizado anteriormente, a condição para que não haja sobreposição entre os retângulos é

$$
\left|\hat{c}_{1}^{i}-\hat{c}_{1}^{j}\right| \geq \frac{a_{p^{i}}^{i}+a_{p^{j}}^{j}}{2} \quad \text { ou } \quad\left|\hat{c}_{2}^{i}-\hat{c}_{2}^{j}\right| \geq \frac{b_{p^{i}}^{i}+b_{p^{j}}^{j}}{2}
$$

para $i, j=1, \ldots, m$ e $i \neq j$. Agora, os vértices do retângulo $i$ são dados por

$$
\begin{aligned}
V_{\mathrm{Sw}}^{i, \theta} \equiv V_{\mathrm{sw}}^{i}\left(R^{i}, C^{i}, \theta, p^{i}\right) & =T(\theta)\left(-a_{p^{i}}^{i} / 2,-b_{p^{i}}^{i} / 2\right)+C^{i} \\
V_{\mathrm{Se}}^{i, \theta} & \equiv V_{\mathrm{se}}^{i}\left(R^{i}, C^{i}, \theta, p^{i}\right)=T(\theta)\left(a_{p^{i}}^{i} / 2,-b_{p^{i}}^{i} / 2\right)+C^{i} \\
V_{\mathrm{ne}}^{i, \theta} & \equiv V_{\mathrm{ne}}^{i}\left(R^{i}, C^{i}, \theta, p^{i}\right)=T(\theta)\left(a_{p^{i}}^{i} / 2, b_{p^{i}}^{i} / 2\right)+C^{i} \mathrm{e} \\
V_{\mathrm{nw}}^{i, \theta} \equiv V_{\mathrm{nw}}^{i}\left(R^{i}, C^{i}, \theta, p^{i}\right) & =T(\theta)\left(-a_{p^{i}}^{i} / 2, b_{p^{i}}^{i} / 2\right)+C^{i} .
\end{aligned}
$$


Explicitamente, os vértices $V_{\mathrm{sw}}^{i, \theta}, V_{\mathrm{se}}^{i, \theta}, V_{\mathrm{ne}}^{i, \theta}$ e $V_{\mathrm{nw}}^{i, \theta}$ do retângulo $i$ são dados por

$$
\begin{aligned}
& {\left[V_{\mathrm{sw}}^{i, \theta}\right]_{x}=-\cos (\theta)\left(p^{i} a+\left(1-p^{i}\right) b\right) / 2+\sin (\theta)\left(p^{i} b+\left(1-p^{i}\right) a\right) / 2+c_{1}^{i}} \\
& {\left[V_{\mathrm{sw}}^{i, \theta}\right]_{y}=-\sin (\theta)\left(p^{i} a+\left(1-p^{i}\right) b\right) / 2-\cos (\theta)\left(p^{i} b+\left(1-p^{i}\right) a\right) / 2+c_{2}^{i}} \\
& {\left[V_{\mathrm{se}}^{i, \theta}\right]_{x}=\cos (\theta)\left(p^{i} a+\left(1-p^{i}\right) b\right) / 2+\sin (\theta)\left(p^{i} b+\left(1-p^{i}\right) a\right) / 2+c_{1}^{i}} \\
& {\left[V_{\mathrm{se}}^{i, \theta}\right]_{y}=\sin (\theta)\left(p^{i} a+\left(1-p^{i}\right) b\right) / 2-\cos (\theta)\left(p^{i} b+\left(1-p^{i}\right) a\right) / 2+c_{2}^{i}} \\
& {\left[V_{\mathrm{ne}}^{i, \theta}\right]_{x}=\cos (\theta)\left(p^{i} a+\left(1-p^{i}\right) b\right) / 2-\sin (\theta)\left(p^{i} b+\left(1-p^{i}\right) a\right) / 2+c_{1}^{i}} \\
& {\left[V_{\mathrm{ne}}^{i, \theta}\right]_{y}=\sin (\theta)\left(p^{i} a+\left(1-p^{i}\right) b\right) / 2+\cos (\theta)\left(p^{i} b+\left(1-p^{i}\right) a\right) / 2+c_{2}^{i}} \\
& {\left[V_{\mathrm{nw}}^{i, \theta}\right]_{x}=-\cos (\theta)\left(p^{i} a+\left(1-p^{i}\right) b\right) / 2-\sin (\theta)\left(p^{i} b+\left(1-p^{i}\right) a\right) / 2+c_{1}^{i}} \\
& {\left[V_{\mathrm{nw}}^{i, \theta}\right]_{y}=-\sin (\theta)\left(p^{i} a+\left(1-p^{i}\right) b\right) / 2+\cos (\theta)\left(p^{i} b+\left(1-p^{i}\right) a\right) / 2+c_{2}^{i}}
\end{aligned}
$$

Novamente, formulando o problema EOR como um problema de otimização, desejamos

$$
\begin{array}{ll}
\text { minimizar } & f_{2}(C, p, \theta) \\
\text { sujeita a } & V_{d}^{i, \theta} \in \Omega_{1}, i=1, \ldots, m \text { e } d \in D \\
& p \in\{0,1\}^{m}
\end{array}
$$

onde

$$
\begin{aligned}
f_{2}(C, p, \theta)=\sum_{i=1}^{m-1} \sum_{j=i+1}^{m} \max \{0, & {\left[p^{i} a+\left(1-p^{i}\right) b+p^{j} a+\left(1-p^{j}\right) b\right]^{2} / 4-} \\
& {\left.\left[\left(c_{1}^{i} \cos (-\theta)-c_{2}^{i} \sin (-\theta)\right)-\left(c_{1}^{j} \cos (-\theta)-c_{2}^{j} \sin (-\theta)\right)\right]^{2}\right\}^{2} } \\
\times \max \left\{0, \quad\left[p^{i} b+\left(1-p^{i}\right) a+p^{j} b+\left(1-p^{j}\right) a\right]^{2} / 4-\right. & \\
& {\left.\left[\left(c_{1}^{i} \sin (-\theta)+c_{2}^{i} \cos (-\theta)\right)-\left(c_{1}^{j} \sin (-\theta)+c_{2}^{j} \cos (-\theta)\right)\right]^{2}\right\}^{2} } \\
+\sum_{k=1}^{r} \sum_{i=1}^{m} \sum_{d \in D} \max \{0, & \left.g_{k}\left(V_{d}^{i, \theta}\right)\right\}^{2} .
\end{aligned}
$$

Pelo fato de nosso método ser capaz de lidar com restrições gerais no nível inferior, podemos remover o termo $\sum_{k=1}^{r} \sum_{i=1}^{m} \sum_{d \in D} \max \left\{0, g_{k}\left(V_{d}^{i, \theta}\right)\right\}^{2}$ da função objetivo e introduzi-lo na forma de restrições do nível inferior do programa. Assim, obtemos o seguinte programa:

$$
\begin{array}{ll}
\text { minimizar } & f_{3}(C, p, \theta) \\
\text { sujeita a } & g_{k}\left(V_{d}^{i, \theta}\right) \leq 0, \quad i=1, \ldots, m, k=1, \ldots, r \text { e } d \in D \\
& V_{d}^{i, \theta} \in \Omega_{1}, \quad i=1, \ldots, m \text { e } d \in D \\
& p \in\{0,1\}^{m}
\end{array}
$$


onde

$$
\begin{aligned}
f_{3}(C, p, \theta)=\sum_{i=1}^{m-1} \sum_{j=i+1}^{m} \max \{0, & {\left[p^{i} a+\left(1-p^{i}\right) b+p^{j} a+\left(1-p^{j}\right) b\right]^{2} / 4-} \\
& {\left.\left[\left(c_{1}^{i} \cos (-\theta)-c_{2}^{i} \sin (-\theta)\right)-\left(c_{1}^{j} \cos (-\theta)-c_{2}^{j} \sin (-\theta)\right)\right]^{2}\right\}^{2} } \\
\times \max \{0, & {\left[p^{i} b+\left(1-p^{i}\right) a+p^{j} b+\left(1-p^{j}\right) a\right]^{2} / 4-} \\
& {\left.\left[\left(c_{1}^{i} \sin (-\theta)+c_{2}^{i} \cos (-\theta)\right)-\left(c_{1}^{j} \sin (-\theta)+c_{2}^{j} \cos (-\theta)\right)\right]^{2}\right\}^{2} . }
\end{aligned}
$$

\subsection{3 Ângulo de rotação}

O posicionamento de um retângulo $i$ em relação aos eixos cartesianos é determinado por uma combinação do ângulo de rotação $\theta$ e da variável $p^{i}$. A posição do retângulo também depende, é claro, de seu centro $C^{i}$, mas o nosso interesse é analisar apenas a rotação do retângulo.

$\theta$ é uma variável que naturalmente não precisa estar limitada. Qualquer valor que ela assuma pode ser convertido em outro pertencente ao intervalo $[0,2 \pi]$, de forma que a solução encontrada seja a mesma. Mais ainda, como estamos tratando da rotação de um retângulo, podemos nos limitar ao caso em que $\theta \in[0, \pi]$, já que após uma rotação de 180 graus o retângulo volta à sua posição inicial.

Se considerarmos ainda uma rotação adicional de 90 graus que não dependa de $\theta$, podemos, também, nos concentrarmos em encontrar $\theta$ no intervalo $[0, \pi / 2]$. No modelo apresentado na Seção 5.2.2, a variável $p^{i}$ é responsável por essa rotação adicional (do retângulo $i$ ). Sendo assim, consideraremos três casos: $\theta \in[0, \pi / 2], \theta \in[0, \pi]$ e $\theta \in$ $(-\infty,+\infty)$

Se restringirmos $\theta$ ao intervalo $[0, \pi]$, é possível obter todas as rotações do retângulo $i$ independentemente do valor de $p^{i}$.

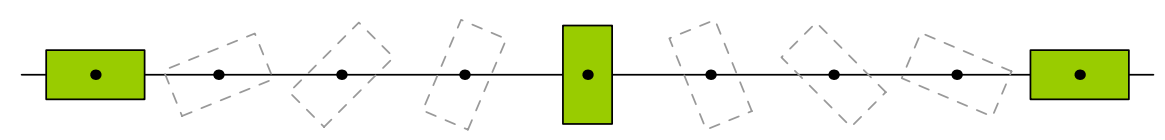

(a) $p^{i}=0$.

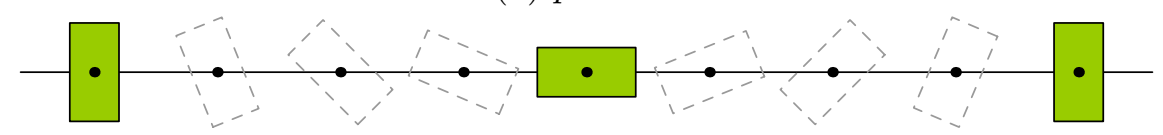

(b) $p^{i}=1$.

Figura 5.7: Para $\theta \in[0, \pi]$, é possível obter qualquer rotação de forma independente do valor de $p^{i}$.

Por outro lado, se restringirmos $\theta$ ao intervalo [0, $\pi / 2$ ], o posicionamento do retângulo $i$ dependerá de uma combinação de $\theta$ e da variável $p^{i}$. A Figura 5.8 ilustra as possíveis posições do retângulo $i$. Note que o primeiro caso, em que $p^{i}=0$, complementa as posições 


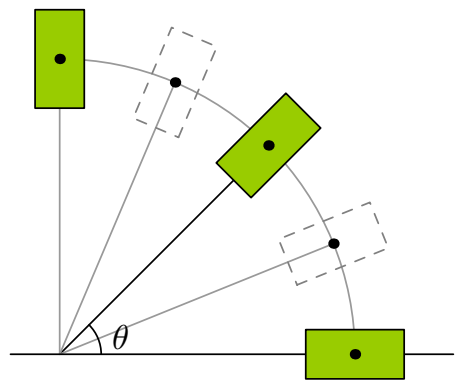

(a) $p^{i}=0$.

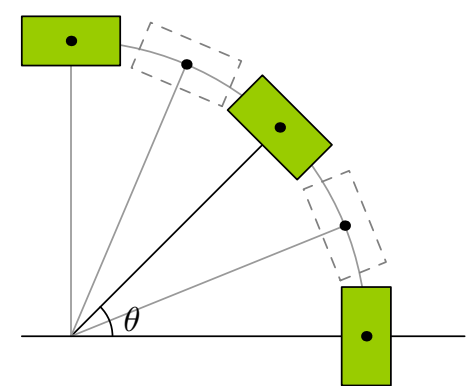

(b) $p^{i}=1$.

Figura 5.8: Possíveis posições do retângulo $i$ considerando $\theta \in[0, \pi / 2]$. Em (a), o caso em que $p^{i}=0$, e, em (b), o caso em que $p^{i}=1$.

fornecidas no segundo caso, em que $p^{i}=1$. Dessa forma, obtemos todas as posições intermediárias que aparecem no movimento de rotação de 180 graus do retângulo.

Um outra possibilidade é não restringir o intervalo da variável $\theta$, deixando-a livre. Em princípio, essa deveria ser a opção preferencial, visto que a introdução de limites em uma variável pode produzir pontos estacionários que não são minimizadores ou maximizadores.

Para exemplificar esse fenômeno, considere o problema de minimizar a função sen $\theta$. As soluções para esse problema são da forma $3 \pi / 2+2 k \pi$ para $k$ inteiro. Assim, qualquer intervalo de tamanho $2 \pi$ contém uma solução ótima para o problema. Se estivermos interessados em qualquer uma das soluções ótimas para esse problema, podemos, por exemplo, restringir $\theta$ ao intervalo $[0,2 \pi]$ e obter o seguinte problema:

$$
\begin{array}{ll}
\text { minimizar } & \operatorname{sen} \theta \\
\text { sujeita a } & \theta \in[0,2 \pi] .
\end{array}
$$

Esse problema possui quatro pontos estacionários, como mostra a Figura 5.9a. Dois deles são pontos da fronteira, onde o gradiente projetado da função objetivo é nulo, um é um minimizador e o outro é um maximizador. Contudo, se restringirmos $\theta$ ao intervalo

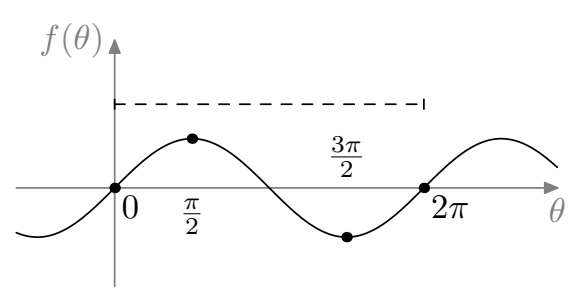

(a) $\theta \in[0,2 \pi]$.

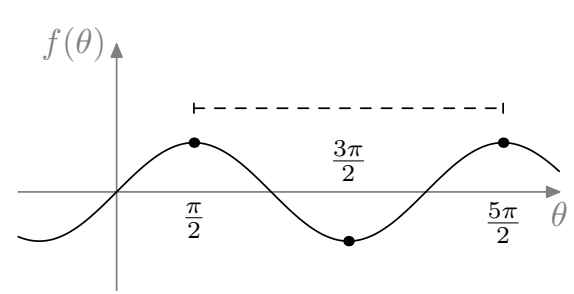

(b) $\theta \in\left[\frac{\pi}{2}, \frac{5 \pi}{2}\right]$.

Figura 5.9: Exemplo da introdução de pontos estacionários com a mudança nos limites da variável $\theta$. Em (a), temos um problema com quatro pontos estacionários. Em (b), temos um problema com apenas três pontos estacionários.

$[\pi / 2,5 \pi / 2]$, temos um problema com um ponto estacionário a menos, como mostra a Figura 5.9b. 


\subsubsection{Ponto inicial}

Pelo fato do problema definido em (5.17) não ser convexo, precisaremos usar técnicas apropriadas para a resolução de problemas não-convexos, a fim de conseguirmos soluções com boa qualidade. Podemos, por exemplo, usar a estratégia de reinícios para tentar resolver o problema. Como essa estratégia é baseada na geração de diversos pontos iniciais e posterior resolução do problema, podemos definir uma forma mais adequada do que a simples geração de pontos aleatórios.

Como as variáveis $p^{i}$ para $i=1, \ldots, m$ são binárias, é muito natural que a escolha de seus valores seja feita no intervalo $[0,1]$. Para a variável $\theta$, podemos tomar valores no intervalo $[0, \pi]$ por exemplo. Já para a escolha dos valores das variáveis que definem os centros dos retângulos, isto é, das variáveis $C^{i}$ para $i=1, \ldots, m$, apresentamos algumas possibilidades a seguir.

A primeira opção é selecionar pontos que estejam próximos da região convexa que define o problema que estamos interessados em resolver. Para isso, podemos definir uma caixa que contenha a região convexa. Em particular, podemos usar a menor caixa que contém a região convexa, que pode ser obtida da seguinte forma. Sejam

$$
\begin{gathered}
g_{1}\left(x_{1}, x_{2}\right) \leq 0 \\
g_{2}\left(x_{1}, x_{2}\right) \leq 0 \\
\vdots \\
g_{r}\left(x_{1}, x_{2}\right) \leq 0
\end{gathered}
$$

as inequações que definem a região convexa no plano. Seja $\Omega_{\mathrm{c}}=\left\{x \in \mathbb{R}^{2} \mid l \leq x \leq u\right\}$ a menor caixa que contém a região definida por (5.19). Os limites $l$ e $u$ da caixa são dados por

$$
\begin{aligned}
l_{1} & =\arg \min \left\{x_{1}: g_{k}\left(x_{1}, x_{2}\right) \leq 0, k=1, \ldots, r\right\}, \\
l_{2} & =\arg \min \left\{x_{2}: g_{k}\left(x_{1}, x_{2}\right) \leq 0, k=1, \ldots, r\right\}, \\
u_{1} & =\arg \max \left\{x_{1}: g_{k}\left(x_{1}, x_{2}\right) \leq 0, k=1, \ldots, r\right\}, \\
u_{2} & =\arg \max \left\{x_{2}: g_{k}\left(x_{1}, x_{2}\right) \leq 0, k=1, \ldots, r\right\} .
\end{aligned}
$$

Um ponto inicial para o problema pode ser dado por um ponto aleatório dentro desta caixa, que é facilmente calculado.

Uma tentativa para melhorar a qualidade do ponto inicial é produzir pontos que estejam dentro da região convexa que define o problema. Para isso, podemos simplesmente gerar pontos dentro da caixa, como anteriormente, e verificar se eles pertencem à região. Se um determinado ponto não pertencer à região convexa, basta tomar, aleatoriamente, um outro ponto dentro da caixa e repetir esse processo até encontrar um ponto que pertença à região convexa. Note que, até aqui, estamos pedindo que o centro do retângulo esteja dentro da região convexa. Uma outra possibilidade é exigir que o retângulo inteiro esteja dentro da região convexa. Para isso, basta gerar pontos que façam com que os vértices dos retângulos estejam dentro da região convexa.

Uma técnica mais sofisticada, e que promete fornecer pontos inicias com qualidade superior, é baseada em empacotamento de círculos. Essa técnica foi usada na implementação 


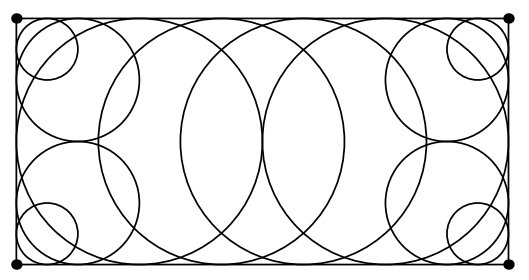

Figura 5.10: Exemplo de cobertura de um retângulo por círculos.

do método descrito em [17] e foi determinante para que o método tivesse um bom desempenho computacional. A idéia é cobrir parcialmente (porém quase totalmente) cada um dos retângulos com círculos e resolver o problema de empacotar tais círculos dentro da região convexa sem que os círculos de um retângulo se sobreponham aos círculos dos demais retângulos. A grande vantagem é que este problema é mais fácil do que o problema de empacotar retângulos e, ainda que uma solução para este problema não forneça uma solução para o problema (5.17), ela pode fornecer um ponto inicial muito bom para se resolver o problema (5.17).

Sejam $a$ o tamanho do lado horizontal e $b$ o tamanho do lado vertical de um retângulo (com $a \geq b$ ). Inicialmente, para garantir que o retângulo representado pelos círculos permaneça dentro da região convexa, círculos com raios desprezíveis são colocados em cada um dos vértices do retângulo. Em seguida, quatro círculos com raios de tamanho $\frac{b}{8}$ são colocados em cada um dos quatro cantos do retângulo, sendo cada um dos círculos tangente aos dois lados adjacentes do retângulo. Outros quatro círculos com raios de tamanho $\frac{b}{4}$ também são colocados da mesma forma.

Adicionalmente, $2\left\lfloor\frac{a}{b}\right\rfloor$ círculos com raios de tamanho $\frac{b}{2}$ são igualmente distribuídos de um lado ao outro do retângulo, como ilustra a Figura 5.10. Considerando o retângulo com o seu centro sobre a origem do plano cartesiano, o primeiro desses círculos será colocado no lado esquerdo do retângulo, tendo seu centro na posição $\left(\frac{b-a}{2}, 0\right)$, o último será colocado no lado direito do retângulo, com seu centro na posição $\left(\frac{a-b}{2}, 0\right)$, e os demais serão colocados entre esses dois e igualmente espaçados de $\frac{a-b}{2\left\lfloor\frac{a}{b}\right\rfloor-1}$. Assim, ordenando esses círculos em ordem crescente da coordenada horizontal do centro, o $j$-ésimo círculo terá a coordenada vertical do seu centro em 0 e a coordenada horizontal em

$$
\frac{b-a}{2}+(j-1) \frac{a-b}{2\left\lfloor\frac{a}{b}\right\rfloor-1} .
$$

Ao contrário do problema resolvido em [17], em que os tamanhos do lados dos retângulos são fixos, nós lidamos com subproblemas nos quais os tamanhos dos lados de cada retângulo são determinados por uma variável (a saber, $p^{i}$ ), como mostra (5.15). Apesar de $p^{i}$ ser uma variável binária, essa restrição é removida na relaxação dos subproblemas de forma que $p^{i}$ passa a ser uma variável contínua entre 0 e 1 . Nesses subproblemas, a menos que a variável $p^{i}$ esteja fixada em 0 ou 1 , o $i$-ésimo retângulo pode ter o tamanho de seus lados variando continuamente entre $b$ e $a$. Não podemos, então, simplesmente definir uma cobertura por círculos considerando o retângulo em sua dimensão original, mas sim defi- 


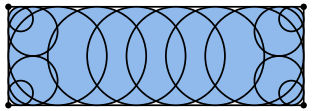

$p^{i}=1$.

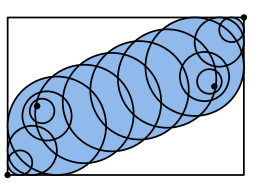

$p^{i}=0.7$.

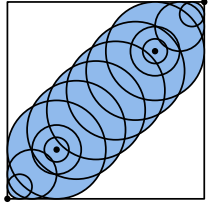

$p^{i}=0.5$.

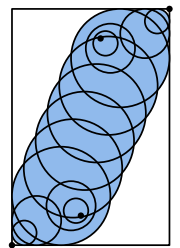

$p^{i}=0.3$.

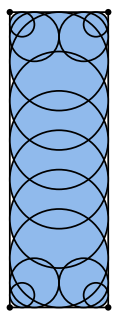

$p^{i}=0$.

Figura 5.11: Distribuição dos círculos dentro do retângulo com seus centros dados por (5.20).

nir uma cobertura para cada um dos possíveis retângulos dados por (5.15). Além disso, também precisamos levar em consideração a rotação do retângulo.

Consideremos a cobertura por círculos como explicado acima para o retângulo em seu tamanho original. De maneira similar ao que foi feito em (5.15) para determinar as dimensões do retângulo, podemos definir uma forma de obter a posição dos círculos em um retângulo com suas dimensões dadas por (5.15) a partir da posição dos círculos do retângulo em seu tamanho original. Seja $C_{c_{k}}$ a constante que indica o centro do $k$-ésimo círculo do retângulo quando o retângulo tem o seu centro localizado na origem do plano cartesiano e o seu tamanho original, isto é, $(a, b)$. Assim, dada uma posição qualquer $\left(p^{i}, C^{i}, \theta\right)$ do retângulo $i$, o centro $\bar{C}_{c_{k}}^{i}$ do $k$-ésimo círculo do retângulo $i$ pode ser dado por

$$
\bar{C}_{c_{k}}^{i}=T(\theta)\left(\begin{array}{c}
p^{i}\left[C_{c_{k}}\right]_{x}+\left(1-p^{i}\right)\left[C_{c_{k}}\right]_{y} \\
p^{i}\left[C_{c_{k}}\right]_{y}+\left(1-p^{i}\right)\left[C_{c_{k}}\right]_{x}
\end{array}\right)+C^{i} .
$$

Apesar de obtermos o resultado desejado quando $p^{i}=0$ e $p^{i}=1$, o centro do círculo dado por (5.20) produz alguns resultados não satisfatórios (do ponto de vista da cobertura do retângulo) para valores intermediários de $p^{i}$, como ilustra a Figura 5.11. Podemos observar que uma grande área do retângulo fica descoberta para $p^{i}=0.5$, por exemplo. De fato, experimentos preliminares mostraram que resolver este problema de empacotamento de círculos não produz bons pontos iniciais para o problema de empacotamento de retângulos, o que já era esperado com base na ilustração da Figura 5.11. Para evitar esse tipo de comportamento, definiremos, a seguir, a disposição dos círculos individualmente para cada retângulo e não apenas com o aproveitamento da cobertura do retângulo original.

Consideremos o retângulo $i$ de dimensões $\left(a_{p^{i}}^{i}, b_{p^{i}}^{i}\right)$, conforme (5.15). Da mesma forma como explicado anteriormente, colocaremos três círculos de raios $0, \frac{b}{8}$ e $\frac{b}{4}$ em cada um dos cantos do retângulo. Tendo em mente a deformação do retângulo causada por $p^{i}$, modificaremos a disposição e a quantidade dos demais círculos. Considerando o retângulo $i$ posicionado na origem do plano cartesiano e sem rotação, pretendemos não apenas distribuir círculos horizontalmente do lado esquerdo ao lado direito do retângulo, mas também verticalmente, do lado de baixo ao lado de cima. Seja $r$ o raio destes círculos e seja $2 N$ o número de círculos a serem colocados ( $N$ horizontalmente, $N$ verticalmente e 
$N \geq 1$ ). Os círculos na horizontal sempre terão a coordenada vertical do centro em 0 e os círculos na vertical terão sempre a coordenada horizontal em 0. Para que os círculos permaneçam inteiramente dentro do retângulo, precisamos exigir que o raio destes círculos satisfaça

$$
r \leq \frac{1}{2} \min \left\{a_{p^{i}}^{i}, b_{p^{i}}^{i}\right\} .
$$

Vamos tratar primeiro dos círculos dispostos horizontalmente. Se $N=1$, o círculo será colocado no ponto $(0,0)$. Senão, colocaremos um círculo no ponto $\left(\frac{1}{2}\left(2 r-a_{p^{i}}^{i}\right), 0\right)$ e outro no ponto $\left(\frac{1}{2}\left(a_{p^{i}}^{i}-2 r\right), 0\right)$. Se $N>2$, colocaremos os demais círculos entre os dois anteriores e igualmente espaçados de $\Delta_{x}$, onde

$$
\Delta_{x}=\frac{a_{p^{i}}^{i}-2 r}{N-1}
$$

Assim, as coordenadas dos centros destes círculos terão a forma $\left(x_{j}, 0\right)$ com

$$
x_{j}=\frac{2 r-a_{p^{i}}^{i}}{2}+(j-1) \Delta_{x}, \quad j=2, \ldots, N .
$$

De forma análoga, para os círculos dispostos verticalmente, teremos

$$
\Delta_{y}=\frac{b_{p^{i}}^{i}-2 r}{N-1}
$$

e as coordenadas dos centros destes círculos terão a forma $\left(0, y_{j}\right)$ com

$$
y_{j}=\frac{2 r-b_{p^{i}}^{i}}{2}+(j-1) \Delta_{y}, \quad j=1, \ldots, N .
$$

Definidos os centros $C_{c_{k}}^{i}$ dos círculos do retângulo $i$ desta maneira, podemos calcular os centros $\bar{C}_{c_{k}}^{i}$ destes círculos relativamente a $\left(p^{i}, C^{i}, \theta\right)$ da seguinte forma

$$
\bar{C}_{c_{k}}^{i}=T(\theta) C_{c_{k}}^{i}+C^{i} .
$$

A Figura 5.12 exemplifica a cobertura do retângulo neste caso, para $(a, b)=(3,1)$ e considerando $r=b$ e $N=2$.

Para que esses círculos tangenciem lados opostos do retângulo e cubram uma parte maior do retângulo, precisamos tomar $r=\frac{1}{2} \min \left\{a_{p^{i}}^{i}, b_{p^{i}}^{i}\right\}$. Neste caso, no entanto, $r$ seria uma variável e da forma como está definida causaria alguns problemas relacionados à não diferenciablidade da função objetivo, como veremos adiante. Para evitar problemas deste tipo, podemos simplesmente tomar $r=\frac{b}{2}$, que é a maior constante que satisfaz (5.21).

Um modelo para o problema de empacotamento de círculos pode ser então obtido simplesmente substituindo, na função objetivo do modelo (5.17) (ou do modelo (5.18)), o termo responsável pela sobreposição de retângulos por um termo capaz de avaliar a sobreposição dos círculos.

A sobreposição entre dois círculos é facilmente calculada. Sejam $\left(x_{1}, y_{1}\right)$ e $r_{1}$ o centro e o raio de um círculo $A$ e $\left(x_{2}, y_{2}\right)$ e $r_{2}$ o centro e o raio de um círculo $B$. Para que os 


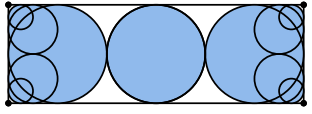

$p^{i}=1$.

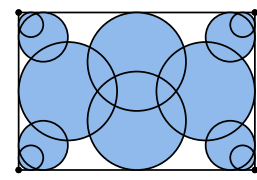

$p^{i}=0.7$.

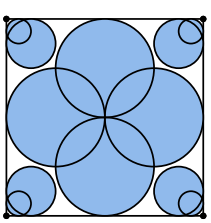

$p^{i}=0.5$.

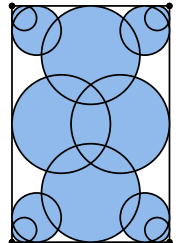

$p^{i}=0.3$.

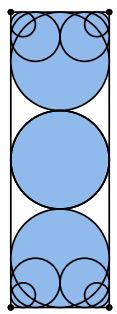

$p^{i}=0$

Figura 5.12: Distribuição dos círculos dentro do retângulo com seus centros dados por (5.22).

círculos $A$ e $B$ não se sobreponham, a distância entre os centros dos círculos não deve ser menor do que a soma de seus raios. Ou seja, devemos ter

$$
\sqrt{\left(x_{1}-x_{2}\right)^{2}+\left(y_{1}-y_{2}\right)^{2}} \geq r_{1}+r_{2}
$$

para que os círculos $A$ e $B$ não se sobreponham. Equivalentemente, podemos exigir que

$$
\left(r_{1}+r_{2}\right)^{2}-\left(x_{1}-x_{2}\right)^{2}-\left(y_{1}-y_{2}\right)^{2} \leq 0 .
$$

Agora, podemos desenvolver um termo para penalizar a sobreposição entre os círculos. Seja $r_{c_{k}}^{i}$ o centro do $k$-ésimo círculo do retângulo $i$. Então, para que o $k$-ésimo círculo do retângulo $i$ não se sobreponha ao $l$-ésimo círculo do retângulo $j$ devemos ter

$$
\left(r_{c_{k}}^{i}+r_{c_{l}}^{j}\right)^{2}-\left(\left[\bar{C}_{c_{k}}^{i}\right]_{x}-\left[\bar{C}_{c_{l}}^{j}\right]_{x}\right)^{2}-\left(\left[\bar{C}_{c_{k}}^{i}\right]_{y}-\left[\bar{C}_{c_{l}}^{j}\right]_{y}\right)^{2} \leq 0
$$

e podemos penalizar essa restrição com

$$
\max \left\{0,\left(r_{c_{k}}^{i}+r_{c_{l}}^{j}\right)^{2}-\left(\left[\bar{C}_{c_{k}}^{i}\right]_{x}-\left[\bar{C}_{c_{l}}^{j}\right]_{x}\right)^{2}-\left(\left[\bar{C}_{c_{k}}^{i}\right]_{y}-\left[\bar{C}_{c_{l}}^{j}\right]_{y}\right)^{2}\right\}^{2} .
$$

Seja $z$ o número de círculos presentes em cada retângulo. Logo, o termo responsável pela sobreposição dos retângulos na função objetivo do modelo (5.17) deve ser substituído por

$$
\sum_{i=1}^{m-1} \sum_{j=i+1}^{m} \sum_{k=1}^{z} \sum_{l=1}^{z} \max \left\{0,\left(r_{c_{k}}^{i}+r_{c_{l}}^{j}\right)^{2}-\left(\left[\bar{C}_{c_{k}}^{i}\right]_{x}-\left[\bar{C}_{c_{l}}^{j}\right]_{x}\right)^{2}-\left(\left[\bar{C}_{c_{k}}^{i}\right]_{y}-\left[\bar{C}_{c_{l}}^{j}\right]_{y}\right)^{2}\right\}^{2} .
$$

\subsection{Experimentos computacionais}

Nesta seção, apresentamos os experimentos realizados e os resultados obtidos por nosso método para resolver o problema de empacotamento ortogonal de retângulos com rotação, descrito na Seção 5.2. Optamos por utilizar os modelos (5.17) e (5.18) apresentados na Seção 5.2.2, já que eles proporcionam problemas mais adequados para serem resolvidos usando o nosso método.

Esses modelos foram implementados de duas formas: a primeira delas usando a linguagem de modelagem AMPL [34] e a segunda em Fortran. No primeiro caso, as derivadas são 
calculadas por diferenciação automática pela biblioteca do AMPL [38] (amplsolver.a). Já no segundo caso, tanto as primeiras quanto as segundas derivadas da função objetivo e das restrições foram calculadas manualmente e então escritas em Fortran. Realizamos uma comparação com o método de diferenças finitas centrais implementado pelo ALGENCAN para nos certificarmos de que as derivadas foram calculadas corretamente.

Todos os testes foram executados em um computador 2.4GHz Intel Core2 Quad Q6600 com 4.0GB de memória RAM e Sistema Operacional Linux. O programa foi compilado com o compilador GNU Fortran (GCC) 4.2.1 e a opção -O3 de otimização de código foi adotada.

As instâncias usadas em nossos testes são as mesmas que foram usadas por Birgin et al. [18]. A Tabela 5.1 contém as definições de cada uma das instâncias. A primeira coluna mostra o número do problema, a segunda coluna exibe as funções que definem a região convexa do problema, a terceira coluna mostra uma estimativa para a área da região convexa (que pode ser usada para calcular um limitante superior para o número de retângulos que podem ser empacotados dentro da região), e as duas últimas colunas mostram as dimensões e a área dos retângulos, respectivamente.

Como estratégia para o método branch and bound, usamos pseudo-custos para determinar a variável de divisão, busca em profundidade e a estratégia de reinícios, já que os problemas de empacotamento considerados possuem muitos mínimos locais. A escolha dessas estratégias foi baseada nos resultados de experimentos preliminares.

Apesar de conhecermos previamente um limitante inferior para cada um dos problemas, executamos o programa para resolver os problemas começando com apenas um retângulo e incrementamos esse número a cada vez que o algoritmo encontrava uma solução. Assim, o tempo reportado nas tabelas a seguir refere-se ao tempo total gasto para resolver desde o problema com um único retângulo até o problema com o número máximo de retângulos encontrado.

Para compararmos tanto a qualidade da solução encontrada quanto o tempo gasto para encontrar a solução, compilamos e executamos em nossa máquina as implementações dos métodos apresentados em [17] e [18]. A Tabela 5.2 mostra os resultados. Na primeira coluna temos o número do problema e na segunda coluna temos um limitante superior dado pela razão entre a área da região convexa e a área do retângulo. Na terceira coluna temos o número de retângulos empacotados pelo método que considera rotações arbitrárias dos retângulos [17] e na quarta coluna temos o tempo gasto por esse método para encontrar a solução. Os mesmos dados são apresentados nas duas últimas colunas para o método que considera apenas rotações ortogonais [18]. Os dados correspondentes ao problema 11 não estão disponíveis para o método introduzido em [17].

As demais tabelas estão organizadas no seguinte formato. A primeira coluna exibe o número do problema. A segunda coluna mostra o número de retângulos empacotados no seguinte formato

$$
n_{\mathrm{r}}\left[d_{\mathrm{a}}, d_{\mathrm{o}}\right]
$$

onde $n_{\mathrm{r}}$ é o número de retângulos empacotados pelo método em questão, $d_{\mathrm{a}}$ é a diferença entre $n_{\mathrm{r}}$ e o número de retângulos empacotados pelo método de rotações arbitrárias introduzido em [17] e $d_{\mathrm{o}}$ é a diferença entre $n_{\mathrm{r}}$ e o número de retângulos empacotados pelo 


\begin{tabular}{|c|c|c|c|c|}
\hline \multirow{2}{*}{ Problema } & \multicolumn{2}{|l|}{ Região convexa } & \multicolumn{2}{|c|}{ Retângulo } \\
\hline & Descrição & Área & Dimensões & Área \\
\hline 1 & $\begin{array}{l}g_{1}\left(x_{1}, x_{2}\right)=-x_{1} \\
g_{2}\left(x_{1}, x_{2}\right)=-x_{2} \\
g_{3}\left(x_{1}, x_{2}\right)=-x_{1}-x_{2}+3 \\
g_{4}\left(x_{1}, x_{2}\right)=x_{1}^{2}+x_{2}^{2}-100\end{array}$ & 74.1 & $2 \times 1$ & 2 \\
\hline 2 & $\begin{array}{l}g_{1}\left(x_{1}, x_{2}\right)=-7 x_{1}+6 x_{2}-24 \\
g_{2}\left(x_{1}, x_{2}\right)=7 x_{1}+6 x_{2}-108 \\
g_{3}\left(x_{1}, x_{2}\right)=\left(x_{1}-6\right)^{2}+\left(x_{2}-8\right)^{2}-9\end{array}$ & 21.7 & $1.1 \times 0.55$ & 0.61 \\
\hline 3 & $\begin{array}{l}g_{1}\left(x_{1}, x_{2}\right)=-x_{1} \\
g_{2}\left(x_{1}, x_{2}\right)=x_{1}-8 \\
g_{3}\left(x_{1}, x_{2}\right)=\left(x_{1}-6\right)^{2}+x_{2}^{2}-81 \\
g_{4}\left(x_{1}, x_{2}\right)=\left(x_{1}-1.7\right)^{2}+\left(x_{2}-10\right)^{2}-81\end{array}$ & 54.4 & $2 \times 0.6$ & 1.2 \\
\hline 4 & $\begin{array}{l}g_{1}\left(x_{1}, x_{2}\right)=x_{1}^{2}-x_{2} \\
g_{2}\left(x_{1}, x_{2}\right)=x_{1}^{2} / 4+x_{2}-5\end{array}$ & 13.3 & $1 \times 0.4$ & 0.40 \\
\hline 5 & $\begin{array}{l}g_{1}\left(x_{1}, x_{2}\right)=x_{1}^{2}-x_{2} \\
g_{2}\left(x_{1}, x_{2}\right)=-x_{1}+x_{2}^{2}-6 x_{2}+6 \\
g_{3}\left(x_{1}, x_{2}\right)=x_{1}+x_{2}-6\end{array}$ & 10.9 & $0.9 \times 0.3$ & 0.27 \\
\hline 6 & $\begin{array}{l}g_{1}\left(x_{1}, x_{2}\right)=-x_{1}+x_{2}^{2}-6 x_{2}+6 \\
g_{2}\left(x_{1}, x_{2}\right)=x_{1}+x_{2}^{2}-3 x_{2}-3 / 4\end{array}$ & 10.2 & $0.9 \times 0.3$ & 0.27 \\
\hline 7 & $g_{1}\left(x_{1}, x_{2}\right)=\left(x_{1}-2\right)^{2} / 4+\left(x_{2}-4\right)^{2} / 16-1$ & 25.1 & $2 \times 0.5$ & 1 \\
\hline 8 & $\begin{array}{l}g_{1}\left(x_{1}, x_{2}\right)=\left(x_{1}-6\right)^{2} / 4+\left(x_{2}-6\right)^{2} / 36-1 \\
g_{2}\left(x_{1}, x_{2}\right)=\left(x_{1}-6\right)^{2} / 36+\left(x_{2}-6\right)^{2} / 4-1 \\
g_{3}\left(x_{1}, x_{2}\right)=x_{1}-x_{2}-3 \\
g_{4}\left(x_{1}, x_{2}\right)=-x_{1}+x_{2}-2\end{array}$ & 13.2 & $0.7 \times 0.5$ & 0.35 \\
\hline 9 & $\begin{array}{l}g_{1}\left(x_{1}, x_{2}\right)=\left(x_{1}-3\right)^{2} / 4+\left(x_{2}-4\right)^{2} / 16-1 \\
g_{2}\left(x_{1}, x_{2}\right)=\left(x_{1}-2.65\right)^{2} / 4+\left(x_{2}-4\right)^{2} / 16-1 \\
g_{3}\left(x_{1}, x_{2}\right)=-x_{1}+1 \\
g_{4}\left(x_{1}, x_{2}\right)=x_{1}-x_{2}-1 \\
g_{5}\left(x_{1}, x_{2}\right)=x_{1}+x_{2}-9\end{array}$ & 13.7 & $0.8 \times 0.6$ & 0.48 \\
\hline 10 & $\begin{array}{l}g_{1}\left(x_{1}, x_{2}\right)=\left(x_{1}-6\right)^{2} / 36+\left(x_{2}-6\right)^{2} / 4-1 \\
g_{2}\left(x_{1}, x_{2}\right)=\left(x_{1}-6\right)^{2} / 9+\left(x_{2}-8\right)^{2} / 9-1\end{array}$ & 13.6 & $0.95 \times 0.35$ & 0.33 \\
\hline 11 & $\begin{array}{l}g_{1}\left(x_{1}, x_{2}\right)=\left(x_{1} / 6\right)^{4}+\left(x_{2} / 2\right)^{4}-1 \\
g_{2}\left(x_{1}, x_{2}\right)=8 x_{1}-11 x_{2}-26\end{array}$ & 34.7 & $1.9 \times 0.5$ & 0.95 \\
\hline 12 & $\begin{array}{l}g_{1}\left(x_{1}, x_{2}\right)=\sqrt{3} x_{1}+x_{2}-\sqrt{3}(4+8 / \sqrt{3}) \\
g_{2}\left(x_{1}, x_{2}\right)=-\sqrt{3} x_{1}+x_{2} \\
g_{3}\left(x_{1}, x_{2}\right)=-x_{2}\end{array}$ & 32.2 & $1 \times 1$ & 1 \\
\hline 13 & $\begin{array}{l}g_{1}\left(x_{1}, x_{2}\right)=\sqrt{3} x_{1}+x_{2}-\sqrt{3}(3+10 / \sqrt{3}) \\
g_{2}\left(x_{1}, x_{2}\right)=-\sqrt{3} x_{1}+x_{2} \\
g_{3}\left(x_{1}, x_{2}\right)=-x_{2}\end{array}$ & 33.3 & $1 \times 1$ & 1 \\
\hline 14 & $\begin{array}{l}g_{1}\left(x_{1}, x_{2}\right)=\sqrt{3} x_{1}+x_{2}-\sqrt{3}(8+2 / \sqrt{3}) \\
g_{2}\left(x_{1}, x_{2}\right)=-\sqrt{3} x_{1}+x_{2} \\
g_{3}\left(x_{1}, x_{2}\right)=-x_{2}\end{array}$ & 36.3 & $1 \times 1$ & 1 \\
\hline 15 & $\begin{array}{l}g_{1}\left(x_{1}, x_{2}\right)=\sqrt{3} x_{1}+x_{2}-\sqrt{3}(9.302) \\
g_{2}\left(x_{1}, x_{2}\right)=-\sqrt{3} x_{1}+x_{2} \\
g_{3}\left(x_{1}, x_{2}\right)=-x_{2}\end{array}$ & 37.5 & $1 \times 1$ & 1 \\
\hline 16 & $\begin{array}{l}g_{1}\left(x_{1}, x_{2}\right)=\sqrt{3} x_{1}+x_{2}-\sqrt{3}(7+4 / \sqrt{3}) \\
g_{2}\left(x_{1}, x_{2}\right)=-\sqrt{3} x_{1}+x_{2} \\
g_{3}\left(x_{1}, x_{2}\right)=-x_{2}\end{array}$ & 37.5 & $1 \times 1$ & 1 \\
\hline
\end{tabular}

Tabela 5.1: Definição dos problemas.

método descrito em [18]. Se $n_{\mathrm{r}}$ estiver em negrito então isso significa que a solução encontrada é melhor do que as soluções encontradas pelos métodos em [17] e [18]. Como a região viável do problema tratado em [18] está incluída na região viável do problema introduzido neste capítulo, podemos esperar que o nosso método encontre soluções tão 


\begin{tabular}{|c|c|c|c|c|c|}
\hline \multirow[b]{2}{*}{ Problema } & \multirow[b]{2}{*}{$\begin{array}{l}\text { Limitante } \\
\text { superior }\end{array}$} & \multicolumn{2}{|c|}{ Rotações arbitrárias [17] } & \multicolumn{2}{|c|}{ Rotações ortogonais [18] } \\
\hline & & $\begin{array}{c}\text { Retângulos } \\
\text { empacotados }\end{array}$ & $\begin{array}{l}\text { Tempo em } \\
\text { segundos }\end{array}$ & $\begin{array}{c}\text { Retângulos } \\
\text { empacotados }\end{array}$ & $\begin{array}{l}\text { Tempo em } \\
\text { segundos }\end{array}$ \\
\hline 1 & 37 & 32 & 147729.78 & 32 & 3131.90 \\
\hline 2 & 35 & 30 & 610.53 & 28 & 14.52 \\
\hline 3 & 45 & 37 & 18020.96 & 40 & 12738.61 \\
\hline 4 & 33 & 28 & 2549.49 & 26 & 295.21 \\
\hline 5 & 40 & 35 & 53073.28 & 33 & 247.52 \\
\hline 6 & 37 & 32 & 4090.31 & 30 & 607.48 \\
\hline 7 & 25 & 20 & 367.74 & 19 & 100.25 \\
\hline 8 & 37 & 32 & 280.50 & 32 & 1023.65 \\
\hline 9 & 28 & 24 & 336.05 & 22 & 65.15 \\
\hline 10 & 40 & 33 & 6110.95 & 34 & 365.09 \\
\hline 11 & 36 & - & - & 31 & 39096.27 \\
\hline 12 & 32 & 27 & 754.37 & 25 & 0.59 \\
\hline 13 & 33 & 28 & 19.25 & 26 & 1.22 \\
\hline 14 & 36 & 29 & 18.69 & 29 & 0.25 \\
\hline 15 & 37 & 30 & 14.63 & 29 & 0.24 \\
\hline 16 & 37 & 31 & 35.78 & 30 & 1.24 \\
\hline
\end{tabular}

Tabela 5.2: Resultados obtidos pelos métodos descritos em [17] e [18].

boas quanto às encontradas pelo método descrito em [18]. Nos casos em que isso não ocorre, indicamos esse fato na primeira coluna da tabela através de um asterisco $\left(^{*}\right)$ ao lado do número do problema. Por fim, a terceira coluna da tabela mostra o número de subproblemas resolvidos (problemas de minimização em caixas) e a última coluna mostra o tempo necessário para encontrar a solução.

Quando fizermos referência a IALGENCAN, estaremos nos referindo ao método descrito na Seção 3.2, capaz de lidar com variáveis inteiras.

\subsection{1 Ângulo de rotação}

O objetivo do primeiro experimento é avaliar a influência dos limites na variável $\theta$. Consideramos os três casos seguintes: $\theta \in[0, \pi / 2], \theta \in[0, \pi]$ e $\theta \in(-\infty, \infty)$. Em nossos experimentos, optamos por usar o modelo (5.17) implementado em Fortran e, para cada um dos três limites em $\theta$ considerados, usamos a estratégia de reinícios com 5 e 100 resoluções de subproblemas por nó da árvore do branch and bound. Além disso, os pontos iniciais são dados pela regra mais simples descrita na Seção 5.2.4, em que os pontos gerados estão próximos à região convexa que define o problema. As Tabelas 5.3 e 5.4 apresentam os resultados para $\theta \in[0, \pi / 2]$, as Tabelas 5.5 e 5.6 apresentam os resultados para $\theta \in[0, \pi]$ e as Tabelas 5.7 e 5.8 mostram os resultados para $\theta \in(-\infty, \infty)$.

Analisaremos, primeiramente, os resultados obtidos com o uso da estratégia de reinícios com 5 resoluções por nó. Antes de mais nada, podemos notar que o nosso método encontrou uma solução de melhor qualidade para o problema de número 8. Enquanto os métodos de rotações arbitrárias e ortogonais encontraram soluções com 32 retângulos para esse problema, nosso método encontrou soluções com um retângulo a mais. No entanto, para 
$\theta \in[0, \pi / 2]$, nosso método encontrou soluções de pior qualidade em 7 dos 16 problemas e de melhor qualidade em 6 problemas se comparado com o método de rotações ortogonais. Já para $\theta \in[0, \pi]$, nosso método perde em qualidade de solução nos problemas $1,3,11$ e 12 quando comparado com o método de rotações ortogonais, e ganha em qualidade em 4 problemas (a saber, nos problemas $2,6,8$ e 9). Para $\theta \in(-\infty, \infty)$ os resultados parecem melhores, sendo que apenas os problemas 1, 3 e 11 exibem soluções de qualidade inferior em relação ao método de rotações ortogonais.

Já com o uso da estratégia de reinícios com 100 resoluções por nó, os resultados melhoram significativamente. Para $\theta \in[0, \pi / 2]$ e $\theta \in[0, \pi]$, apenas os problemas 3 e 11 apresentaram soluções de qualidade inferior às apresentadas pelo método de rotações ortogonais. Para $\theta \in(-\infty, \infty)$, isso ocorre apenas com o problema 11, para o qual o nosso método encontra uma solução com 30 retângulos e o método de rotações ortogonais encontra uma solução com 31 retângulos. Por outro lado, a qualidade das soluções encontradas é superior às encontradas pelo método de rotações ortogonais em 6 problemas (a saber, problemas $2,4,5,6,8$ e 9). Portanto, com exceção do problema 11, para $\theta \in(-\infty, \infty)$ temos um método capaz de encontrar soluções de qualidade superior com um eventual pequeno aumento no consumo de tempo.

Uma vez que que a qualidade das soluções encontradas por nosso método para as diferentes escolhas de limite para $\theta$ varia bastante, uma comparação baseada no tempo de processamento torna-se um pouco complicada. Porém, podemos analisar o impacto no tempo de processamento causado pela mudança na estratégia de reinícios (de 5 para 100 resoluções por nó) considerando apenas os problemas cujas soluções permaneceram com a mesma qualidade. Assim, para $\theta \in[0, \pi / 2]$, o tempo chegou a ficar 11.75 vezes maior (problema 6); para $\theta \in[0, \pi]$ o tempo chegou a ficar 8.5 vezes maior (problema 10); e para $\theta \in(-\infty, \infty)$ o tempo chegou a ficar 10 vezes maior (problema 10).

Vale a pena mencionar que, apesar da região viável do problema de empacotamento considerado neste capítulo estar contida na região viável do problema abordado em [17], nosso método encontrou soluções de melhor qualidade nos problemas 3, 8 e 10 .

Levando-se em consideração a qualidade da solução encontrada, podemos dizer que o modelo em que a variável $\theta$ está livre é o melhor dos três para esse conjunto de instâncias. Nos experimentos seguintes, consideraremos apenas o modelo com a variável $\theta$ livre.

\subsubsection{Ponto inicial}

Pretendemos, agora, comparar as diferentes estratégias de construção de pontos iniciais descritas na Seção 5.2.4. Novamente, optamos por utilizar o modelo (5.17) implementado em Fortran, a estratégia de reinícios com 100 resoluções por nó e $\theta \in(-\infty, \infty)$. A primeira estratégia, e a mais simples, produz pontos iniciais próximos à região convexa do problema, isto é, os centros dos retângulos são gerados dentro da menor caixa que contém a região convexa do problema. Os resultados para essa estratégia são apresentados na Tabela 5.8. Na segunda estratégia, os pontos iniciais são gerados dentro da região convexa. Os resultados são apresentados na Tabela 5.9.

A primeira mudança que observamos é quanto a qualidade das soluções encontradas. Ao passo que com a primeira estratégia pudemos encontrar uma solução com 40 retângulos 


\begin{tabular}{|c|c|c|c|c|}
\hline \multirow[b]{2}{*}{ Problema } & \multicolumn{3}{|c|}{ Número de } & \multirow{2}{*}{$\begin{array}{c}\text { Tempo de } \\
\text { processamento } \\
\text { em segundos }\end{array}$} \\
\hline & \multicolumn{2}{|c|}{$\begin{array}{c}\text { retângulos } \\
\text { empacotados }\end{array}$} & $\begin{array}{l}\text { subproblemas } \\
\text { resolvidos }\end{array}$ & \\
\hline $1^{*}$ & 31 & {$[-1,-1]$} & 566 & 174.86 \\
\hline 2 & 30 & $0,+2]$ & 762 & 161.15 \\
\hline $3^{*}$ & 38 & {$[+1,-2]$} & 1238 & 633.77 \\
\hline 4 & 27 & {$[-1,+1]$} & 750 & 105.79 \\
\hline 5 & 34 & {$[-1,+1]$} & 839 & 329.78 \\
\hline 6 & 32 & $0,+2]$ & 692 & 264.04 \\
\hline 7 & 19 & {$\left[\begin{array}{ll}-1, & 0\end{array}\right]$} & 250 & 12.77 \\
\hline 8 & 33 & {$[+1,+1]$} & 4853 & 1284.54 \\
\hline 9 & 24 & $0,+2]$ & 689 & 43.39 \\
\hline $10^{*}$ & 33 & {$[+1,-1]$} & 1481 & 237.03 \\
\hline $11^{*}$ & 29 & {$[-,-2]$} & 447 & 186.21 \\
\hline $12^{*}$ & 24 & {$[-3,-1]$} & 25 & 1.32 \\
\hline $13^{*}$ & 25 & {$[-3,-1]$} & 27 & 1.53 \\
\hline 14 & 29 & $0, \quad 0]$ & 32 & 3.79 \\
\hline $15^{*}$ & 28 & {$[-2,-1]$} & 28 & 1.62 \\
\hline 16 & 30 & {$[-1,0]$} & 32 & 1.68 \\
\hline
\end{tabular}

Tabela 5.3: Resultados obtidos usando o modelo (5.17) implementado em Fortran, restringindo a variável $\theta$ ao intervalo $[0, \pi / 2]$ e usando a estratégia de reinícios com 5 resoluções por nó.

\begin{tabular}{|c|c|c|c|c|}
\hline \multirow[b]{2}{*}{ Problema } & \multicolumn{3}{|c|}{ Número de } & \multirow{2}{*}{$\begin{array}{c}\text { Tempo de } \\
\text { processamento } \\
\text { em segundos }\end{array}$} \\
\hline & \multicolumn{2}{|c|}{$\begin{array}{c}\text { retângulos } \\
\text { empacotados }\end{array}$} & $\begin{array}{l}\text { subproblemas } \\
\text { resolvidos }\end{array}$ & \\
\hline 1 & 32 & $\left.\begin{array}{ll}0, & 0\end{array}\right]$ & 18305 & 5452.98 \\
\hline 2 & 30 & $0,+2]$ & 2506 & 396.19 \\
\hline $3^{*}$ & 39 & {$[+2,-1]$} & 9283 & 5938.14 \\
\hline 4 & 27 & {$[-1,+1]$} & 2315 & 409.04 \\
\hline 5 & 34 & {$[-1,+1]$} & 7653 & 3186.29 \\
\hline 6 & 32 & $0,+2]$ & 6631 & 3102.96 \\
\hline 7 & 19 & {$\left[\begin{array}{ll}-1, & 0\end{array}\right]$} & 393 & 19.64 \\
\hline 8 & 33 & {$[+1,+1]$} & 27924 & 5478.53 \\
\hline 9 & 24 & $0,+2]$ & 6439 & 387.52 \\
\hline 10 & 34 & {$\left[\begin{array}{ll}+2, & 0\end{array}\right]$} & 18674 & 3342.42 \\
\hline $11^{*}$ & 30 & {$[-,-1]$} & 3276 & 1659.22 \\
\hline 12 & 25 & {$\left[\begin{array}{ll}-2, & 0\end{array}\right]$} & 31 & 1.70 \\
\hline 13 & 26 & {$\left[\begin{array}{ll}-2, & 0\end{array}\right]$} & 34 & 2.59 \\
\hline 14 & 29 & $0, \quad 0]$ & 32 & 3.82 \\
\hline 15 & 29 & {$\left[\begin{array}{ll}-1, & 0\end{array}\right]$} & 34 & 5.70 \\
\hline 16 & 30 & {$\left[\begin{array}{ll}-1, & 0\end{array}\right]$} & 32 & 1.69 \\
\hline
\end{tabular}

Tabela 5.4: Resultados obtidos usando o modelo (5.17) implementado em Fortran, restringindo a variável $\theta$ ao intervalo $[0, \pi / 2]$ e usando a estratégia de reinícios com 100 resoluções por nó. 


\begin{tabular}{|c|c|c|c|c|}
\hline \multirow[b]{2}{*}{ Problema } & \multicolumn{3}{|c|}{ Número de } & \multirow{2}{*}{$\begin{array}{c}\text { Tempo de } \\
\text { processamento } \\
\text { em segundos }\end{array}$} \\
\hline & & $\begin{array}{l}\text { ngulos } \\
\text { icotados }\end{array}$ & $\begin{array}{c}\text { subproblemas } \\
\text { resolvidos }\end{array}$ & \\
\hline $1^{*}$ & 31 & {$[-1,-1]$} & 399 & 121.61 \\
\hline 2 & 30 & $0,+2]$ & 677 & 153.98 \\
\hline $3^{*}$ & 39 & {$[+2,-1]$} & 1124 & 705.76 \\
\hline 4 & 26 & {$[-2, \quad 0]$} & 371 & 21.77 \\
\hline 5 & 33 & {$\left[\begin{array}{ll}-2, & 0\end{array}\right]$} & 1036 & 409.96 \\
\hline 6 & 31 & {$[-1,+1]$} & 519 & 150.88 \\
\hline 7 & 19 & {$\left[\begin{array}{ll}-1, & 0\end{array}\right]$} & 230 & 8.72 \\
\hline 8 & 33 & {$[+1,+1]$} & 2663 & 626.82 \\
\hline 9 & 24 & $0,+2]$ & 1389 & 105.42 \\
\hline 10 & 34 & {$[+2, \quad 0]$} & 1989 & 353.00 \\
\hline $11^{*}$ & 29 & {$[-,-2]$} & 426 & 160.95 \\
\hline $12^{*}$ & 24 & {$[-3,-1]$} & 24 & 0.71 \\
\hline 13 & 26 & {$\left[\begin{array}{ll}-2, & 0\end{array}\right]$} & 29 & 1.64 \\
\hline 14 & 29 & $\left.\begin{array}{ll}0, & 0\end{array}\right]$ & 30 & 1.71 \\
\hline 15 & 29 & {$\left[\begin{array}{ll}-1, & 0\end{array}\right]$} & 30 & 2.57 \\
\hline 16 & 30 & {$\left[\begin{array}{ll}-1, & 0\end{array}\right]$} & 30 & 1.36 \\
\hline
\end{tabular}

Tabela 5.5: Resultados obtidos usando o modelo (5.17) implementado em Fortran, restringindo a variável $\theta$ ao intervalo $[0, \pi]$ e usando a estratégia de reinícios com 5 resoluções por nó.

\begin{tabular}{|c|c|c|c|c|}
\hline \multirow[b]{2}{*}{ Problema } & \multicolumn{3}{|c|}{ Número de } & \multirow{2}{*}{$\begin{array}{c}\text { Tempo de } \\
\text { processamento } \\
\text { em segundos }\end{array}$} \\
\hline & \multicolumn{2}{|c|}{$\begin{array}{c}\text { retângulos } \\
\text { empacotados }\end{array}$} & $\begin{array}{l}\text { subproblemas } \\
\text { resolvidos }\end{array}$ & \\
\hline 1 & 32 & $\left.\begin{array}{ll}0, & 0\end{array}\right]$ & 1164 & 495.04 \\
\hline 2 & 30 & $0,+2]$ & 3498 & 617.86 \\
\hline $3^{*}$ & 39 & {$[+2,-1]$} & 3111 & 1999.24 \\
\hline 4 & 27 & {$[-1,+1]$} & 3083 & 502.11 \\
\hline 5 & 34 & {$[-1,+1]$} & 18092 & 8346.48 \\
\hline 6 & 32 & $0,+2]$ & 5558 & 2392.67 \\
\hline 7 & 19 & {$\left[\begin{array}{ll}-1, & 0\end{array}\right]$} & 253 & 9.29 \\
\hline 8 & 33 & {$[+1,+1]$} & 18840 & 3219.90 \\
\hline 9 & 24 & $0,+2]$ & 11873 & 692.15 \\
\hline 10 & 34 & {$\left[\begin{array}{ll}+2, & 0\end{array}\right]$} & 17892 & 2999.29 \\
\hline $11^{*}$ & 30 & {$[-,-1]$} & 1660 & 807.65 \\
\hline 12 & 25 & {$\left[\begin{array}{ll}-2, & 0\end{array}\right]$} & 33 & 1.46 \\
\hline 13 & 26 & {$\left[\begin{array}{ll}-2, & 0\end{array}\right]$} & 29 & 1.68 \\
\hline 14 & 29 & $0, \quad 0]$ & 30 & 1.69 \\
\hline 15 & 29 & {$\left[\begin{array}{ll}-1, & 0\end{array}\right]$} & 30 & 2.62 \\
\hline 16 & 30 & {$\left[\begin{array}{ll}-1, & 0\end{array}\right]$} & 30 & 1.38 \\
\hline
\end{tabular}

Tabela 5.6: Resultados obtidos usando o modelo (5.17) implementado em Fortran, restringindo a variável $\theta$ ao intervalo $[0, \pi]$ e usando a estratégia de reinícios com 100 resoluções por nó. 


\begin{tabular}{|c|c|c|c|c|}
\hline \multirow[b]{2}{*}{ Problema } & \multicolumn{3}{|c|}{ Número de } & \multirow{2}{*}{$\begin{array}{c}\text { Tempo de } \\
\text { processamento } \\
\text { em segundos }\end{array}$} \\
\hline & \multicolumn{2}{|c|}{$\begin{array}{c}\text { retângulos } \\
\text { empacotados }\end{array}$} & $\begin{array}{c}\text { subproblemas } \\
\text { resolvidos }\end{array}$ & \\
\hline $1^{*}$ & 31 & {$[-1,-1]$} & 418 & 176.73 \\
\hline 2 & 30 & $0,+2]$ & 741 & 94.99 \\
\hline $3^{*}$ & 39 & {$[+2,-1]$} & 955 & 615.41 \\
\hline 4 & 27 & {$[-1,+1]$} & 647 & 94.53 \\
\hline 5 & 33 & {$\left[\begin{array}{ll}-2, & 0\end{array}\right]$} & 886 & 251.49 \\
\hline 6 & 32 & $0,+2]$ & 1558 & 748.04 \\
\hline 7 & 19 & {$\left[\begin{array}{ll}-1, & 0\end{array}\right]$} & 255 & 12.88 \\
\hline 8 & 33 & {$[+1,+1]$} & 3237 & 711.96 \\
\hline 9 & 24 & $0,+2]$ & 1948 & 185.41 \\
\hline 10 & 34 & {$\left[\begin{array}{ll}+2, & 0\end{array}\right]$} & 1304 & 191.01 \\
\hline $11^{*}$ & 30 & {$[-,-1]$} & 600 & 272.38 \\
\hline 12 & 25 & {$\left[\begin{array}{ll}-2, & 0\end{array}\right]$} & 28 & 1.61 \\
\hline 13 & 26 & {$\left[\begin{array}{ll}-2, & 0\end{array}\right]$} & 27 & 1.24 \\
\hline 14 & 29 & $0, \quad 0]$ & 29 & 1.77 \\
\hline 15 & 29 & {$\left[\begin{array}{ll}-1, & 0\end{array}\right]$} & 33 & 4.32 \\
\hline 16 & 30 & {$\left[\begin{array}{ll}-1, & 0\end{array}\right]$} & 32 & 2.88 \\
\hline
\end{tabular}

Tabela 5.7: Resultados obtidos usando o modelo (5.17) implementado em Fortran, deixando livre a variável $\theta$ e usando a estratégia de reinícios com 5 resoluções por nó.

\begin{tabular}{|c|c|c|c|c|}
\hline \multirow[b]{2}{*}{ Problema } & \multicolumn{3}{|c|}{ Número de } & \multirow{2}{*}{$\begin{array}{c}\text { Tempo de } \\
\text { processamento } \\
\text { em segundos }\end{array}$} \\
\hline & \multicolumn{2}{|c|}{$\begin{array}{c}\text { retângulos } \\
\text { empacotados }\end{array}$} & $\begin{array}{c}\text { subproblemas } \\
\text { resolvidos }\end{array}$ & \\
\hline 1 & 32 & $\begin{array}{ll}0, & 0\end{array}$ & 5177 & 1749.95 \\
\hline 2 & 30 & $0,+2]$ & 2614 & 434.28 \\
\hline 3 & 40 & {$[+3,0]$} & 33744 & 19909.84 \\
\hline 4 & 27 & {$[-1,+1]$} & 1635 & 183.32 \\
\hline 5 & 34 & {$[-1,+1]$} & 71803 & 36592.87 \\
\hline 6 & 32 & $0,+2]$ & 4248 & 2195.82 \\
\hline 7 & 19 & {$\left[\begin{array}{ll}-1, & 0\end{array}\right]$} & 758 & 47.32 \\
\hline 8 & 33 & {$[+1,+1]$} & 18480 & 3162.90 \\
\hline 9 & 24 & {$[0,+2]$} & 5628 & 456.71 \\
\hline 10 & 34 & {$[+2,0]$} & 11633 & 1914.92 \\
\hline $11^{*}$ & 30 & {$[-,-1]$} & 907 & 505.55 \\
\hline 12 & 25 & {$\left[\begin{array}{ll}-2, & 0\end{array}\right]$} & 28 & 1.62 \\
\hline 13 & 26 & {$\left[\begin{array}{ll}-2, & 0\end{array}\right]$} & 27 & 1.19 \\
\hline 14 & 29 & $0, \quad 0]$ & 29 & 1.73 \\
\hline 15 & 29 & {$\left[\begin{array}{ll}-1, & 0\end{array}\right]$} & 33 & 4.31 \\
\hline 16 & 30 & {$\left[\begin{array}{ll}-1, & 0\end{array}\right]$} & 32 & 2.88 \\
\hline
\end{tabular}

Tabela 5.8: Resultados obtidos usando o modelo (5.17) implementado em Fortran, deixando livre a variável $\theta$ e usando a estratégia de reinícios com 100 resoluções por nó. 


\begin{tabular}{|c|c|c|c|c|}
\hline \multirow[b]{2}{*}{ Problema } & \multicolumn{3}{|c|}{ Número de } & \multirow{2}{*}{$\begin{array}{c}\text { Tempo de } \\
\text { processamento } \\
\text { em segundos }\end{array}$} \\
\hline & \multicolumn{2}{|c|}{$\begin{array}{c}\text { retângulos } \\
\text { empacotados }\end{array}$} & $\begin{array}{c}\text { subproblemas } \\
\text { resolvidos }\end{array}$ & \\
\hline 1 & 32 & $\left.\begin{array}{ll}0, & 0\end{array}\right]$ & 13818 & 3860.73 \\
\hline 2 & 30 & $0,+2]$ & 8286 & 1239.03 \\
\hline $3^{*}$ & 39 & {$[+2,-1]$} & 3212 & 2229.88 \\
\hline 4 & 27 & {$[-1,+1]$} & 9691 & 649.22 \\
\hline 5 & 33 & {$\left[\begin{array}{ll}-2, & 0\end{array}\right]$} & 21134 & 4848.65 \\
\hline 6 & 32 & $0,+2]$ & 21457 & 6147.67 \\
\hline 7 & 19 & {$[-1,0]$} & 223 & 6.67 \\
\hline 8 & 33 & {$[+1,+1]$} & 17837 & 2852.04 \\
\hline 9 & 24 & $0,+2]$ & 9391 & 471.92 \\
\hline 10 & 34 & {$[+2, \quad 0]$} & 21746 & 3929.00 \\
\hline $11^{*}$ & 30 & {$[-,-1]$} & 3422 & 1267.43 \\
\hline 12 & 25 & {$\left[\begin{array}{ll}-2, & 0\end{array}\right]$} & 42 & 2.56 \\
\hline 13 & 26 & {$\left[\begin{array}{ll}-2, & 0\end{array}\right]$} & 34 & 2.59 \\
\hline 14 & 29 & $\left.\begin{array}{ll}0, & 0\end{array}\right]$ & 29 & 1.75 \\
\hline 15 & 29 & {$\left[\begin{array}{ll}-1, & 0\end{array}\right]$} & 30 & 2.37 \\
\hline 16 & 30 & {$\left[\begin{array}{ll}-1, & 0\end{array}\right]$} & 33 & 3.83 \\
\hline
\end{tabular}

Tabela 5.9: Pontos iniciais gerados dentro da região convexa do problema.

para a instância 3, a segunda estratégia encontrou apenas 39. A qualidade da solução também foi diferente para a instância 5, tendo a primeira estratégia encontrado uma solução com um retângulo a mais. Pelo fato das demais soluções encontradas possuírem a mesma qualidade e tratando-se de duas das instâncias mais difíceis (as instâncias 3 e 5 foram as que consumiram mais tempo para serem resolvidas de acordo com a Tabela 5.8), esse fato por si só não é capaz de indicar uma superioridade por parte da primeira estratégia. Com exceção das instâncias 7,8 e 15, a primeira estratégia foi mais rápida em todas as instâncias. Para a instância 4, por exemplo, o tempo gasto com a segunda estratégia foi cerca de 3.54 vezes maior. É importante observar que o aumento de tempo não foi devido ao tempo gasto para produzir os pontos iniciais dentro da região convexa, mas sim pelo aumento no número de subproblemas. De fato, o número de subproblemas gerados pela segunda estratégia foi maior em 12 instâncias, apesar do tempo médio para se resolver um único subproblema ter diminuído em 10 instâncias. Portanto, a primeira e mais simples estratégia apresentou um desempenho melhor do que a segunda.

Na última estratégia, cada retângulo é parcialmente coberto por círculos e em seguida o problema de empacotar tais círculos na região convexa é resolvido. A solução encontrada é então usada como ponto inicial para o problema de empacotamento de retângulos. A Tabela 5.10 exibe os resultados obtidos com essa estratégia e usando reinícios com 5 resoluções por nó. O número de subproblemas resolvidos apresentado na Tabela 5.10 inclui os subproblemas de empacotamento de círculos. Comparando com os resultados apresentados na Tabela 5.7, que se refere ao método que também usa reinícios com 5 resoluções por nó mas que tem os pontos iniciais dados em uma caixa que contém a região convexa do problema, vemos que as soluções para as instâncias 3 e 11 perdem em qualidade. Para ambas as instâncias, o método com os pontos iniciais dados pelo 


\begin{tabular}{|c|c|c|c|c|}
\hline \multirow[b]{2}{*}{ Problema } & \multicolumn{3}{|c|}{ Número de } & \multirow{2}{*}{$\begin{array}{c}\text { Tempo de } \\
\text { processamento } \\
\text { em segundos }\end{array}$} \\
\hline & \multicolumn{2}{|c|}{$\begin{array}{c}\text { retângulos } \\
\text { empacotados }\end{array}$} & $\begin{array}{c}\text { subproblemas } \\
\text { resolvidos }\end{array}$ & \\
\hline $1^{*}$ & 31 & {$[-1,-1]$} & 1676 & 985.29 \\
\hline 2 & 30 & $0,+2]$ & 2502 & 983.30 \\
\hline $3^{*}$ & 38 & {$[+1,-2]$} & 3002 & 3609.15 \\
\hline 4 & 27 & {$[-1,+1]$} & 2588 & 680.76 \\
\hline 5 & 33 & {$\left[\begin{array}{ll}-2, & 0\end{array}\right]$} & 2204 & 1205.97 \\
\hline 6 & 32 & {$[0,+2]$} & 2916 & 2070.01 \\
\hline 7 & 19 & {$\left[\begin{array}{ll}-1, & 0\end{array}\right]$} & 388 & 34.18 \\
\hline 8 & 33 & {$[+1,+1]$} & 7570 & 2499.82 \\
\hline 9 & 24 & $0,+2]$ & 1432 & 178.17 \\
\hline 10 & 34 & {$[+2,0]$} & 3386 & 891.94 \\
\hline $11^{*}$ & 29 & {$[-,-2]$} & 1170 & 669.32 \\
\hline 12 & 25 & {$\left[\begin{array}{ll}-2, & 0\end{array}\right]$} & 58 & 2.82 \\
\hline 13 & 26 & {$\left[\begin{array}{ll}-2, & 0\end{array}\right]$} & 54 & 2.07 \\
\hline 14 & 29 & $0, \quad 0]$ & 58 & 6.10 \\
\hline 15 & 29 & {$\left[\begin{array}{ll}-1, & 0\end{array}\right]$} & 58 & 2.81 \\
\hline 16 & 30 & {$[-1,0]$} & 60 & 3.18 \\
\hline
\end{tabular}

Tabela 5.10: Pontos iniciais dados pelas soluções do problema de empacotamento de círculos e usando a estratégia de reinícios com 5 resoluções por nó.

empacotamento de círculos encontra soluções com um retângulo a menos. Além disso, o tempo de processamento também é visivelmente muito maior. De fato, exceto para as instâncias 9 e 15, o método com os pontos iniciais dados pela forma mais simples é mais rápido e chega a ser cerca de 10.35 vezes mais rápido para a instância 2 .

\subsubsection{Instâncias $12^{\prime}-16^{\prime}$}

Como podemos observar nas tabelas anteriores, as instâncias 12-16 foram resolvidas com relativa facilidade, isto é, a melhor solução de cada problema foi encontrada rapidamente. Esse baixo esforço computacional também foi observado com o método que considera apenas rotações ortogonais, como podemos ver na Tabela 5.2. Devido a isso, Birgin et al. [18] decidiram construir novas instâncias a partir das instâncias 12-16, simplesmente substituindo o retângulo a ser empacotado por outro com metade da área, com dimensões $1 \times 0.5$. Essas novas instâncias foram chamadas 12'-16'.

Além disso, como as instâncias 12-16 tratam do empacotamento de quadrados, as variáveis inteiras que decidem se cada retângulo ficará "em pé" ou "deitado" não são necessárias. Dessa forma, estas instâncias tornam-se desinteressantes, já que o objetivo do nosso método é resolver problemas com variáveis inteiras. Mais ainda, mesmo para as relaxações contínuas, as dimensões dos retângulos tornam-se constantes de acordo com (5.15), independentemente do valor de $p^{i}$.

Na Tabela 5.11 são apresentados os resultados obtidos pelo método que considera rotações arbitrárias [17], pelo método que considera apenas rotações ortogonais [18] e pelo nosso método para as instâncias 12'-16'. Podemos observar que o nosso método encontrou 


\begin{tabular}{|c|c|c|c|c|c|c|c|}
\hline \multirow[b]{2}{*}{ Problema } & \multirow[b]{2}{*}{$\begin{array}{l}\text { Limitante } \\
\text { superior }\end{array}$} & \multicolumn{2}{|c|}{ Rotações arbitrárias [17] } & \multicolumn{2}{|c|}{ Rotações ortogonais [18] } & \multicolumn{2}{|c|}{ IALGENCAN } \\
\hline & & $\begin{array}{l}\text { Retângulos } \\
\text { empacotados }\end{array}$ & $\begin{array}{l}\text { Tempo em } \\
\text { segundos }\end{array}$ & $\begin{array}{l}\text { Retângulos } \\
\text { empacotados }\end{array}$ & $\begin{array}{l}\text { Tempo em } \\
\text { segundos }\end{array}$ & $\begin{array}{l}\text { Retângulos } \\
\text { empacotados }\end{array}$ & $\begin{array}{l}\text { Tempo em } \\
\text { segundos }\end{array}$ \\
\hline $12^{\prime}$ & 64 & 56 & 33275.87 & 55 & 6975.21 & 55 & 12088.92 \\
\hline $13^{\prime}$ & 66 & 58 & 4550.97 & 57 & 8285.77 & 57 & 3562.25 \\
\hline $14^{\prime}$ & 72 & 64 & 31875.08 & 62 & 10856.04 & 62 & 3185.46 \\
\hline $15^{\prime}$ & 74 & 65 & 5562.10 & 64 & 11406.28 & 64 & 3032.86 \\
\hline $16^{\prime}$ & 74 & 66 & 135864.71 & 64 & 10328.75 & 64 & 3060.99 \\
\hline
\end{tabular}

Tabela 5.11: Resultados obtidos pelos métodos descritos em [17] e [18] para os problemas 12'-16', e pelo nosso método com o modelo (5.17) implementado em Fortran e usando reinícios com 5 resoluções por nó.

soluções equivalentes às encontradas pelo método em [18] e, exceto para a instância 12', o nosso método foi mais rápido em todas as instâncias. Para a instância 13', nosso método foi cerca de 2.32 vezes mais rápido, enquanto que para a instância 15' ele foi cerca de 2.76 vezes mais rápido.

\subsubsection{Fixando as variáveis inteiras}

Como explicado na Seção 4.2, dada uma solução para um subproblema em que as restrições de integralidade são removidas, podemos arredondá-la (isto é, arredondar o valor de cada variável inteira para o número inteiro mais próximo) e verificar se o ponto resultante satisfaz as restrições do subproblema. Seguindo esta mesma idéia, poderíamos ir além do arredondamento e resolver o subproblema novamente, mas desta vez com as variáveis inteiras fixadas em seus valores provenientes do arredondamento. Apesar deste procedimento ser muito mais caro do que o simples arredondamento da solução, podemos eventualmente encontrar soluções inteiras de melhor qualidade.

Na Tabela 5.12 são mostrados os resultados obtidos com esta estratégia. Comparando com os resultados da Tabela 5.8, notamos que houve um decréscimo significativo no tempo de execução para resolver algumas instâncias. A instância 6 foi resolvida em um tempo mais de nove vezes menor e as instâncias 3 e 5 exigiram um tempo mais de sete vezes menor. Por outro lado, a qualidade da solução da instância 11 foi inferior, contendo um retângulo a menos.

\subsubsection{Modelo de empacotamento com restrições}

No modelo (5.17), descrito na Seção 5.2.2, as restrições que exigem que os retângulos permaneçam dentro da região convexa são penalizadas. Examinando a função Lagrangiano Aumentado (3.4), apresentada no Capítulo 3, vemos que penalizar tais restrições da forma como realizado no modelo (5.17) equivale a fixar $\mu=0$ e $\rho=2 \mathrm{em} \mathrm{(3.4).} \mathrm{A} \mathrm{técnica} \mathrm{de} \mathrm{fixar}$ o parâmetro de penalidade é conhecida como "atalho" (ver shortcut em [28], página 285). Em princípio, essas restrições deveriam ser mantidas como restrições de nível inferior no modelo e o método de Lagrangianos Aumentados deveria ser o responsável por penalizá-las e decidir os valores de $\mu$ e $\rho$. Isso é feito no modelo (5.18), que mantém essas restrições 


\begin{tabular}{|c|c|c|c|c|}
\hline \multirow[b]{2}{*}{ Problema } & \multicolumn{3}{|c|}{ Número de } & \multirow{2}{*}{$\begin{array}{c}\text { Tempo de } \\
\text { processamento } \\
\text { em segundos }\end{array}$} \\
\hline & \multicolumn{2}{|c|}{$\begin{array}{c}\text { retângulos } \\
\text { empacotados }\end{array}$} & $\begin{array}{c}\text { subproblemas } \\
\text { resolvidos }\end{array}$ & \\
\hline 1 & 32 & $\left.\begin{array}{ll}0, & 0\end{array}\right]$ & 19271 & 4777.73 \\
\hline 2 & 30 & $0,+2]$ & 174 & 100.02 \\
\hline 3 & 40 & {$[+3,0]$} & 5628 & 2522.34 \\
\hline 4 & 27 & {$[-1,+1]$} & 132 & 23.02 \\
\hline 5 & 34 & {$[-1,+1]$} & 12566 & 5048.98 \\
\hline 6 & 32 & $0,+2]$ & 517 & 235.35 \\
\hline 7 & 19 & {$[-1,0]$} & 46 & 1.69 \\
\hline 8 & 33 & {$[+1,+1]$} & 33798 & 5129.16 \\
\hline 9 & 24 & $0,+2]$ & 151 & 13.94 \\
\hline 10 & 34 & {$[+2, \quad 0]$} & 1934 & 384.47 \\
\hline $11^{*}$ & 29 & {$[-,-2]$} & 119 & 35.03 \\
\hline 12 & 25 & {$\left[\begin{array}{ll}-2, & 0\end{array}\right]$} & 56 & 1.62 \\
\hline 13 & 26 & {$\left[\begin{array}{ll}-2, & 0\end{array}\right]$} & 54 & 1.21 \\
\hline 14 & 29 & $\left.\begin{array}{ll}0, & 0\end{array}\right]$ & 58 & 1.80 \\
\hline 15 & 29 & {$\left[\begin{array}{ll}-1, & 0\end{array}\right]$} & 62 & 4.23 \\
\hline 16 & 30 & {$\left[\begin{array}{ll}-1, & 0\end{array}\right]$} & 64 & 2.94 \\
\hline
\end{tabular}

Tabela 5.12: Resultados obtidos usando reinícios com 100 resoluções por nó e resolvendo o subproblema novamente com as variáveis inteiras fixadas.

no nível inferior.

Nas Tabelas 5.13 e 5.14, temos os resultados obtidos com o modelo (5.18) usando reinícios com 5 e 100 resoluções por nó, respectivamente. Comparando os resultados da Tabela 5.14 com os resultados apresentados na Tabela 5.8, podemos ver que, apesar do tempo médio para se resolver um único subproblema ter sido reduzido em todas as instâncias (uma redução de 12.5 vezes na instância 7, por exemplo), o aumento na quantidade de subproblemas resolvidos foi suficiente para que o tempo de processamento fosse muito maior. Com exceção da instância 7, que foi resolvida em 25.62 segundos, todas as instâncias necessitaram de um tempo maior para serem resolvidas. O tempo para se resolver a instância 4 , em particular, aumentou 6.74 vezes e o tempo para se resolver a instância 13 aumentou cerca de 26.43 vezes. No que diz respeito à qualidade das soluções, notamos uma diferença apenas para a instância 11. Com o modelo (5.17), pudemos encontrar uma solução com 30 retângulos para a instância 7, ao passo que, com o modelo (5.18), encontramos uma solução com um retângulo a menos. Com base nesses resultados, podemos concluir que o modelo (5.17) é o mais adequado para esse conjunto de instâncias.

\subsubsection{Relaxando o corte}

A razão pela qual usamos a estratégia de reinícios vem da não-convexidade da função objetivo do modelo (5.17), que proporciona o aparecimento de diversos mínimos locais. Como o método branch and bound baseia-se no fato de que os subproblemas (quando precisam ser resolvidos) devem ser resolvidos de forma ótima, a estratégia de reinícios tem por objetivo aumentar a chance de se encontrar a solução global do subproblema. 


\begin{tabular}{|c|c|c|c|c|}
\hline \multirow[b]{2}{*}{ Problema } & \multicolumn{3}{|c|}{ Número de } & \multirow{2}{*}{$\begin{array}{c}\text { Tempo de } \\
\text { processamento } \\
\text { em segundos }\end{array}$} \\
\hline & \multicolumn{2}{|c|}{$\begin{array}{c}\text { retângulos } \\
\text { empacotados }\end{array}$} & $\begin{array}{c}\text { subproblemas } \\
\text { resolvidos }\end{array}$ & \\
\hline $1^{*}$ & 31 & {$[-1,-1]$} & 1329 & 94.62 \\
\hline 2 & 30 & $0,+2]$ & 1314 & 151.38 \\
\hline $3^{*}$ & 39 & {$[+2,-1]$} & 2905 & 1031.55 \\
\hline 4 & 27 & {$[-1,+1]$} & 1355 & 124.87 \\
\hline 5 & 33 & {$\left[\begin{array}{ll}-2, & 0\end{array}\right]$} & 2209 & 659.22 \\
\hline 6 & 31 & {$[-1,+1]$} & 1690 & 207.93 \\
\hline 7 & 19 & {$\left[\begin{array}{ll}-1, & 0\end{array}\right]$} & 567 & 11.32 \\
\hline 8 & 33 & {$[+1,+1]$} & 2965 & 507.25 \\
\hline 9 & 24 & $0,+2]$ & 1415 & 122.33 \\
\hline 10 & 34 & {$[+2, \quad 0]$} & 4686 & 850.08 \\
\hline $11^{*}$ & 30 & {$[-,-1]$} & 1573 & 115.37 \\
\hline 12 & 25 & {$\left[\begin{array}{ll}-2, & 0\end{array}\right]$} & 162 & 3.52 \\
\hline 13 & 26 & {$\left[\begin{array}{ll}-2, & 0\end{array}\right]$} & 181 & 3.88 \\
\hline 14 & 29 & $\left.\begin{array}{ll}0, & 0\end{array}\right]$ & 194 & 4.43 \\
\hline 15 & 29 & {$\left[\begin{array}{ll}-1, & 0\end{array}\right]$} & 200 & 3.82 \\
\hline 16 & 30 & {$\left[\begin{array}{ll}-1, & 0\end{array}\right]$} & 228 & 6.01 \\
\hline
\end{tabular}

Tabela 5.13: Modelo (5.18) com restrições, implementado em Fortran e usando reinícios com 5 resoluções por nó.

\begin{tabular}{|c|c|c|c|c|}
\hline \multirow[b]{2}{*}{ Problema } & \multicolumn{3}{|c|}{ Número de } & \multirow{2}{*}{$\begin{array}{c}\text { Tempo de } \\
\text { processamento } \\
\text { em segundos }\end{array}$} \\
\hline & \multicolumn{2}{|c|}{$\begin{array}{c}\text { retângulos } \\
\text { empacotados }\end{array}$} & $\begin{array}{c}\text { subproblemas } \\
\text { resolvidos }\end{array}$ & \\
\hline 1 & 32 & $\left.\begin{array}{ll}0, & 0\end{array}\right]$ & 48369 & 9524.95 \\
\hline 2 & 30 & $0,+2]$ & 26450 & 563.75 \\
\hline 3 & 40 & {$[+3, \quad 0]$} & 52317 & 26597.56 \\
\hline 4 & 27 & {$[-1,+1]$} & 24102 & 1236.93 \\
\hline 5 & 34 & {$[-1,+1]$} & 36493 & 9526.22 \\
\hline 6 & 32 & $0,+2]$ & 39920 & 4570.13 \\
\hline 7 & 19 & {$\left[\begin{array}{ll}-1, & 0\end{array}\right]$} & 5141 & 25.62 \\
\hline 8 & 33 & {$[+1,+1]$} & 50416 & 4805.94 \\
\hline 9 & 24 & $0,+2]$ & 16378 & 540.39 \\
\hline 10 & 34 & {$[+2, \quad 0]$} & 47855 & 2975.81 \\
\hline $11^{*}$ & 29 & {$[-,-2]$} & 22662 & 533.68 \\
\hline 12 & 25 & {$\left[\begin{array}{ll}-2, & 0\end{array}\right]$} & 1676 & 31.80 \\
\hline 13 & 26 & {$\left[\begin{array}{ll}-2, & 0\end{array}\right]$} & 1873 & 31.46 \\
\hline 14 & 29 & $\left.\begin{array}{ll}0, & 0\end{array}\right]$ & 2153 & 23.82 \\
\hline 15 & 29 & {$\left[\begin{array}{ll}-1, & 0\end{array}\right]$} & 1771 & 22.18 \\
\hline 16 & 30 & {$\left[\begin{array}{ll}-1, & 0\end{array}\right]$} & 2288 & 39.65 \\
\hline
\end{tabular}

Tabela 5.14: Modelo (5.18) com restrições, implementado em Fortran e usando reinícios com 100 resoluções por nó. 


\begin{tabular}{|c|c|c|c|c|}
\hline \multirow[b]{2}{*}{ Problema } & \multicolumn{3}{|c|}{ Número de } & \multirow{2}{*}{$\begin{array}{c}\text { Tempo de } \\
\text { processamento } \\
\text { em segundos }\end{array}$} \\
\hline & \multicolumn{2}{|c|}{$\begin{array}{c}\text { retângulos } \\
\text { empacotados }\end{array}$} & $\begin{array}{c}\text { subproblemas } \\
\text { resolvidos }\end{array}$ & \\
\hline 1 & 32 & $\left.\begin{array}{ll}0, & 0\end{array}\right]$ & 40322 & 10574.47 \\
\hline 2 & 30 & $0,+2]$ & 741 & 94.78 \\
\hline 3 & 40 & {$[+3,0]$} & 1484548 & 622073.00 \\
\hline 4 & 28 & {$[0,+2]$} & 3092728 & 304674.76 \\
\hline 5 & 34 & {$[-1,+1]$} & 12015 & 4953.22 \\
\hline 6 & 32 & $0,+2]$ & 2628 & 1056.20 \\
\hline 7 & 19 & {$[-1,0]$} & 189 & 6.21 \\
\hline 8 & 33 & {$[+1,+1]$} & 12891 & 1754.43 \\
\hline 9 & 24 & $0,+2]$ & 828 & 47.45 \\
\hline 10 & 34 & {$[+2, \quad 0]$} & 1848 & 276.47 \\
\hline 11 & 31 & {$\left[\begin{array}{ll}-, & 0\end{array}\right]$} & 251869 & 64298.39 \\
\hline 12 & 25 & {$\left[\begin{array}{ll}-2, & 0\end{array}\right]$} & 28 & 1.60 \\
\hline 13 & 26 & {$\left[\begin{array}{ll}-2, & 0\end{array}\right]$} & 27 & 1.27 \\
\hline 14 & 29 & $\left.\begin{array}{ll}0, & 0\end{array}\right]$ & 29 & 1.78 \\
\hline 15 & 29 & {$\left[\begin{array}{ll}-1, & 0\end{array}\right]$} & 33 & 4.34 \\
\hline 16 & 30 & {$\left[\begin{array}{ll}-1, & 0\end{array}\right]$} & 32 & 2.89 \\
\hline
\end{tabular}

Tabela 5.15: Resultados obtidos usando o modelo (5.17) implementado em Fortran, deixando livre a variável $\theta$, usando a estratégia de reinícios com 5 resoluções por nó, $\alpha=10^{-4}$ e $M=0$.

Além de reinícios, podemos usar também a estratégia de relaxação do corte discutida na Seção 3.2.4, cuja idéia é não cortar ramos da árvore que tenham uma chance maior de conter a solução ótima do problema.

Usando como parâmetros para essa estratégia $\alpha=10^{-4}$ e $M=0$, e aliada à estratégia de reinícios (com 5 resoluções por nó), a Tabela 5.15 mostra os resultados obtidos. Comparando com os resultados obtidos com a nossa melhor estratégia até agora (Tabela 5.8), vemos que as soluções das instâncias 4 e 11 foram melhoradas. Uma atenção especial deve ser dada ao resultado obtido para a instância 11. Até agora, nenhuma das alternativas consideradas tinha sido capaz de encontrar uma solução com 31 retângulos para a instância 11, e esta era a única instância cuja solução dada pelo método de rotações ortogonais [18] era superior.

Na Tabela 5.16, também apresentamos os resultados com a estratégia de relaxação de corte com $\alpha=10^{-4}$ e $M=0$, mas, desta vez, usando reinícios com 100 resoluções por nó. Na Tabela 5.17 são apresentados os resultados com essa mesma estratégia, além do uso da estratégia de fixar as variáveis inteiras conforme explicado na Seção 5.3.4. Comparando os resultados das Tabelas 5.15, 5.16 e 5.17, observamos que as soluções encontradas são igualmente boas. No que diz respeito ao tempo de resolução dos problemas, os resultados da Tabela 5.17 são, em geral, melhores.

Vale a pena mencionar que testes preliminares mostraram que, usando $M=0 \mathrm{e}$ $\alpha \in\left\{10^{-1}, 10^{-2}, 10^{-3}\right\}$, não foi possível encontrar uma solução com 31 retângulos para a instância 11. Isso ocorreu porque esses valores de $\alpha$ não foram suficientes para impedir que o algoritmo cortasse ramos da árvore contendo uma solução para o problema. 


\begin{tabular}{|c|c|c|c|c|}
\hline \multirow[b]{2}{*}{ Problema } & \multicolumn{3}{|c|}{ Número de } & \multirow{2}{*}{$\begin{array}{c}\text { Tempo de } \\
\text { processamento } \\
\text { em segundos }\end{array}$} \\
\hline & \multicolumn{2}{|c|}{$\begin{array}{c}\text { retângulos } \\
\text { empacotados }\end{array}$} & $\begin{array}{c}\text { subproblemas } \\
\text { resolvidos }\end{array}$ & \\
\hline 1 & 32 & $\left.\begin{array}{ll}0, & 0\end{array}\right]$ & 15115 & 4381.78 \\
\hline 2 & 30 & $0,+2]$ & 2614 & 433.34 \\
\hline 3 & 40 & {$[+3, \quad 0]$} & 925640 & 399946.02 \\
\hline 4 & 28 & $0,+2]$ & 5491287 & 584599.93 \\
\hline 5 & 34 & {$[-1,+1]$} & 13199 & 6004.22 \\
\hline 6 & 32 & $0,+2]$ & 5348 & 2686.31 \\
\hline 7 & 19 & {$\left[\begin{array}{ll}-1, & 0\end{array}\right]$} & 758 & 47.37 \\
\hline 8 & 33 & {$[+1,+1]$} & 80880 & 10341.03 \\
\hline 9 & 24 & $0,+2]$ & 11988 & 705.02 \\
\hline 10 & 34 & {$[+2, \quad 0]$} & 11633 & 1878.03 \\
\hline 11 & 31 & {$\left[\begin{array}{ll}-, & 0\end{array}\right]$} & 416100 & 97360.64 \\
\hline 12 & 25 & {$\left[\begin{array}{ll}-2, & 0\end{array}\right]$} & 28 & 1.58 \\
\hline 13 & 26 & {$\left[\begin{array}{ll}-2, & 0\end{array}\right]$} & 27 & 1.21 \\
\hline 14 & 29 & $\left.\begin{array}{ll}0, & 0\end{array}\right]$ & 29 & 1.74 \\
\hline 15 & 29 & {$\left[\begin{array}{ll}-1, & 0\end{array}\right]$} & 33 & 4.30 \\
\hline 16 & 30 & {$\left[\begin{array}{ll}-1, & 0\end{array}\right]$} & 32 & 2.90 \\
\hline
\end{tabular}

Tabela 5.16: Resultados obtidos usando o modelo (5.17) implementado em Fortran, deixando livre a variável $\theta$, usando a estratégia de reinícios com 100 resoluções por nó, $\alpha=10^{-4}$ e $M=0$.

\begin{tabular}{|c|c|c|c|c|}
\hline \multirow[b]{2}{*}{ Problema } & \multicolumn{3}{|c|}{ Número de } & \multirow{2}{*}{$\begin{array}{c}\text { Tempo de } \\
\text { processamento } \\
\text { em segundos }\end{array}$} \\
\hline & \multicolumn{2}{|c|}{$\begin{array}{c}\text { retângulos } \\
\text { empacotados }\end{array}$} & $\begin{array}{c}\text { subproblemas } \\
\text { resolvidos }\end{array}$ & \\
\hline 1 & 32 & $\begin{array}{ll}0, & 0\end{array}$ & 5488 & 1449.31 \\
\hline 2 & 30 & $0,+2]$ & 174 & 97.71 \\
\hline 3 & 40 & {$\left[\begin{array}{ll}+3, & 0\end{array}\right]$} & 150369 & 49937.07 \\
\hline 4 & 28 & $0,+2]$ & 9469327 & 791933.68 \\
\hline 5 & 34 & {$[-1,+1]$} & 185658 & 65363.59 \\
\hline 6 & 32 & $0,+2]$ & 517 & 230.44 \\
\hline 7 & 19 & {$\left[\begin{array}{ll}-1, & 0\end{array}\right]$} & 46 & 1.63 \\
\hline 8 & 33 & {$[+1,+1]$} & 75606 & 8106.83 \\
\hline 9 & 24 & $0,+2]$ & 151 & 13.95 \\
\hline 10 & 34 & {$[+2,0]$} & 1934 & 379.83 \\
\hline 11 & 31 & {$\left[\begin{array}{ll}-, & 0\end{array}\right]$} & 272121 & 48652.84 \\
\hline 12 & 25 & {$\left[\begin{array}{ll}-2, & 0\end{array}\right]$} & 56 & 1.64 \\
\hline 13 & 26 & {$[-2,0]$} & 54 & 1.26 \\
\hline 14 & 29 & $\left.\begin{array}{ll}0, & 0\end{array}\right]$ & 58 & 1.76 \\
\hline 15 & 29 & {$\left[\begin{array}{ll}-1, & 0\end{array}\right]$} & 62 & 4.21 \\
\hline 16 & 30 & {$\left[\begin{array}{ll}-1, & 0\end{array}\right]$} & 64 & 2.88 \\
\hline
\end{tabular}

Tabela 5.17: Resultados obtidos usando o modelo (5.17) implementado em Fortran, deixando livre a variável $\theta$, usando a estratégia de reinícios com 100 resoluções por nó, $\alpha=10^{-4}$ e $M=0$, e resolvendo o subproblema com as variáveis inteiras fixadas. 


\begin{tabular}{|c|c|c|c|c|}
\hline \multirow[b]{2}{*}{ Problema } & \multicolumn{3}{|c|}{ Número de } & \multirow{2}{*}{$\begin{array}{c}\text { Tempo de } \\
\text { processamento } \\
\text { em segundos }\end{array}$} \\
\hline & \multicolumn{2}{|c|}{$\begin{array}{c}\text { retângulos } \\
\text { empacotados }\end{array}$} & $\begin{array}{c}\text { subproblemas } \\
\text { resolvidos }\end{array}$ & \\
\hline 1 & 32 & $\left.\begin{array}{ll}0, & 0\end{array}\right]$ & 39387 & 164288.05 \\
\hline 2 & 30 & $0,+2]$ & 2691 & 2916.31 \\
\hline $3^{*}$ & 39 & {$[+2,-1]$} & 2279 & 18467.84 \\
\hline 4 & 27 & {$[-1,+1]$} & 1541 & 2223.43 \\
\hline 5 & 34 & {$[-1,+1]$} & 13055 & 72684.36 \\
\hline 6 & 32 & {$[0,+2]$} & 1211 & 6361.43 \\
\hline 7 & 19 & {$[-1,0]$} & 353 & 49.72 \\
\hline 8 & 33 & {$[+1,+1]$} & 23713 & 49631.01 \\
\hline 9 & 24 & {$[0,+2]$} & 5483 & 7690.30 \\
\hline 10 & 34 & {$[+2, \quad 0]$} & 16005 & 28295.83 \\
\hline $11^{*}$ & 30 & {$[-,-1]$} & 2718 & 8429.00 \\
\hline 12 & 25 & {$\left[\begin{array}{ll}-2, & 0\end{array}\right]$} & 64 & 30.80 \\
\hline 13 & 26 & {$\left[\begin{array}{ll}-2, & 0\end{array}\right]$} & 54 & 12.50 \\
\hline 14 & 29 & $0, \quad 0]$ & 57 & 7.62 \\
\hline 15 & 29 & {$\left[\begin{array}{ll}-1, & 0\end{array}\right]$} & 58 & 16.05 \\
\hline 16 & 30 & {$\left[\begin{array}{ll}-1, & 0\end{array}\right]$} & 60 & 14.05 \\
\hline
\end{tabular}

Tabela 5.18: Resultados obtidos usando o modelo (5.17) implementado em AMPL usando reinícios com 100 tentativas por nó.

\subsubsection{Modelo em AMPL}

Apenas para dar uma idéia da diferença de desempenho e justificar o trabalho realizado para implementar em Fortran o modelo para o problema de empacotamento de retângulos, mostramos na Tabela 5.18 o resultado obtido ao executarmos nosso programa com o modelo (5.17) implementado em AMPL. Como podemos observar, as soluções encontradas possuem a mesma qualidade das soluções encontradas pelo modelo implementado em Fortran, mas o tempo para resolver os problemas foi muito maior, como pode ser comparado com os resultados apresentados na Tabela 5.8. A única instância que levou menos tempo para ser resolvida foi a instância 3. Ainda assim, com uma diferença de tempo muito pequena. O tempo para se resolver a instância 1, por exemplo, aumentou quase 94 vezes.

\subsubsection{Representações gráficas das soluções}

Apresentamos nesta seção as representações gráficas das soluções encontradas pelo nosso método. Na Figura 5.13, temos uma comparação entre a solução encontrada pelo nosso método e as soluções encontradas pelos métodos descritos em [18] e [17] para a instância 8. Note que o ângulo de rotação dos retângulos na solução exibida na Figura $5.13 \mathrm{~b}$ é bem pequeno em valor absoluto (aproximadamente $-0.02 \mathrm{grau}$ ), mas que com essa pequena mudança foi possível encontrar uma solução melhor do que considerando o ângulo fixado em zero (Figura 5.13a). Na Figura 5.14 temos as soluções das instâncias 12'-16' e na Figura 5.15 temos as soluções das demais instâncias. 


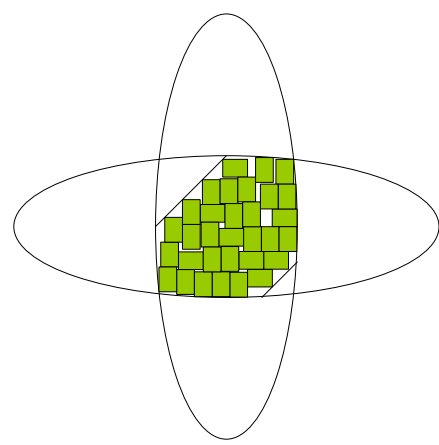

(a)

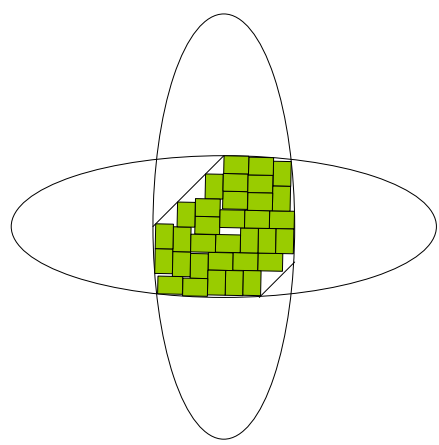

(b)

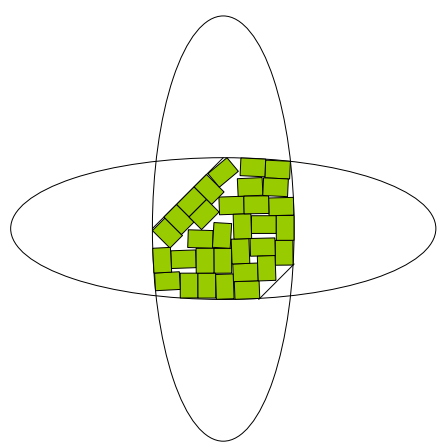

(c)

Figura 5.13: Soluções encontradas para a instância 8. Em (a), a solução encontrada pelo método que considera rotações ortogonais [18] e, em (c), a solução encontrada pelo método que considera rotações arbitrárias [17] (ambas com 32 retângulos). Em (b), a solução encontrada pelo nosso método com 33 retângulos.
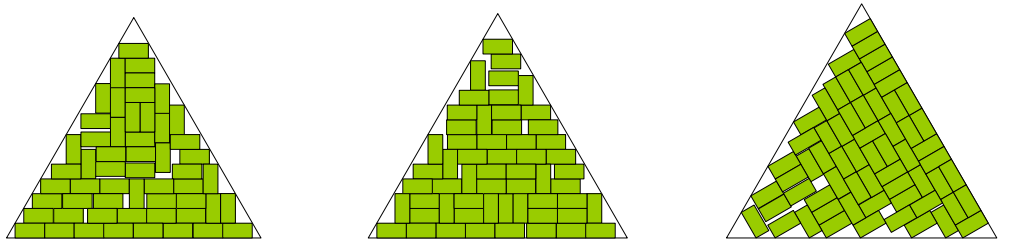

(12') 55 retângulos.

(13') 57 retângulos.

(14') 62 retângulos.

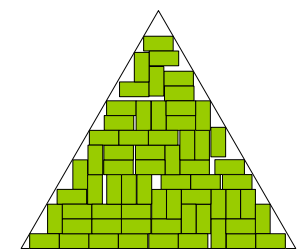

(15’) 64 retângulos.

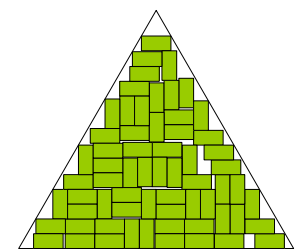

(16’) 64 retângulos.

Figura 5.14: Representação gráfica das soluções encontradas para as instâncias 12'-16'. 


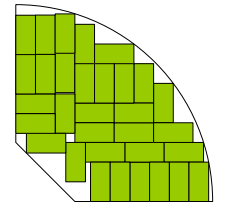

(1) 32 retângulos.

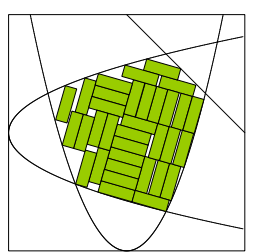

(5) 34 retângulos.

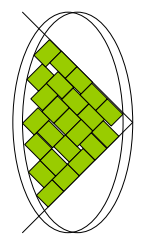

(9) 24 retângulos.

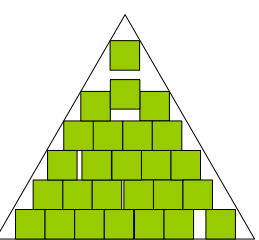

(13) 26 retângulos.

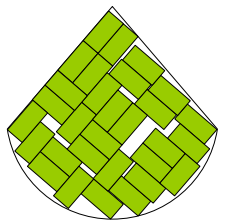

(2) 30 retângulos.

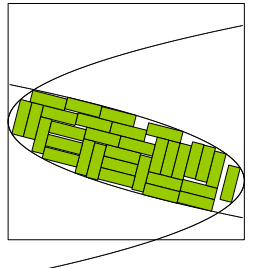

(6) 32 retângulos.

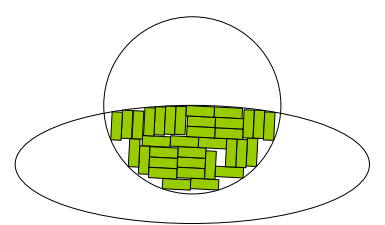

(10) 34 retângulos.

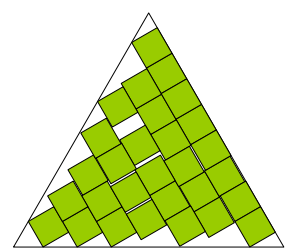

(14) 29 retângulos.

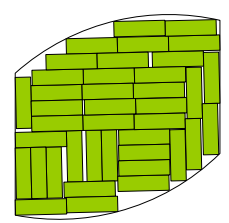

(3) 40 retângulos.

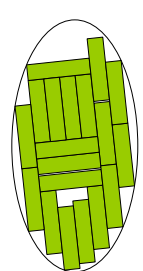

(7) 19 retângulos.

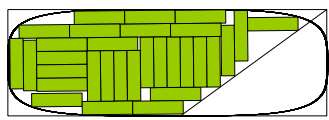

(11) 31 retângulos.

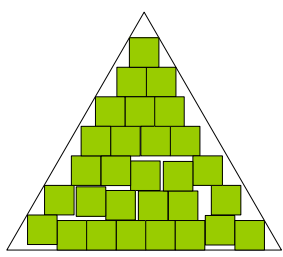

(15) 29 retângulos.

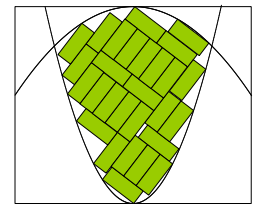

(4) 28 retângulos.

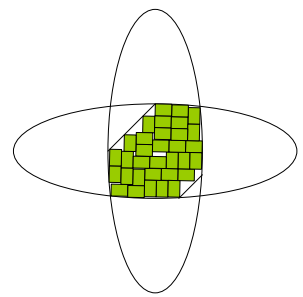

(8) 33 retângulos.

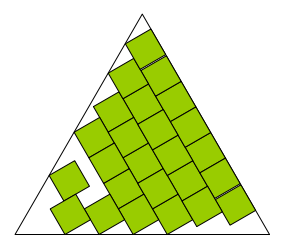

(12) 25 retângulos.

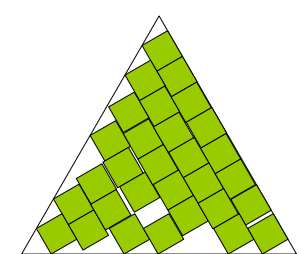

(16) 30 retângulos.

Figura 5.15: Representação gráfica das soluções encontradas para as instâncias 1-16. 


\section{Capítulo 6}

\section{Considerações finais e trabalhos futuros}

O objetivo deste trabalho foi o de desenvolver uma algoritmo para resolver problemas de programação não-linear inteira mista. Tínhamos como base um método de Lagrangianos Aumentados destinado à resolução de problemas de programação não-linear. A idéia era tirar proveito deste método para desenvolver um algoritmo que fosse capaz de resolver MINLPs. Consideramos, inicialmente, um algoritmo baseado em branch and bound e que, em cada nó da árvore, resolve um problema de programação não-linear (resultante da relaxação do subproblema do nó em questão) usando o método de Lagrangianos Aumentados. Chamamos a nossa implementação deste método de Algencan-BB. Neste caso, o método de Lagrangianos Aumentados é apenas usado para resolver as relaxações em cada nó da árvore e poderia ser substituído por qualquer outro algoritmo de programação não-linear.

Posteriormente, consideramos um método de Lagrangianos Aumentados que mantém as restrições de integralidade do MINLP como restrições do nível inferior. Nossa implementação deste método recebeu o nome de IAlgencan. Desse modo, os subproblemas presentes na iteração interna do método de Lagrangianos Aumentados passam a ser problemas de minimização em caixas com variáveis inteiras. Para resolver esses problemas, optamos por usar um método do tipo branch and bound associado à um método adequado para a resolução de problemas de minimização em caixas.

Realizamos experimentos com alguns problemas encontrados na literatura. Os problemas foram obtidos de [31] e da MINLPLib [22]. Para esses problemas, IALGEnCAN mostrou-se muito superior ao ALGENCAN-BB. Em geral, IALGENCAN não apenas foi mais eficiente, mas também foi mais robusto do que Algencan-Bb. Por outro lado, quando comparado com o Bonmin, IAlgencan foi menos robusto, embora mais eficiente. A perda quanto à robustez é explicada pelo fato do IALGENCAN apenas encontrar uma solução local para alguns dos problemas não-convexos. Para contornar isso, poderíamos usar a estratégia de reinícios com um número maior de resoluções de subproblemas por nó, gastando possivelmente um pouco mais de tempo para resolver os problemas.

Contudo, para concluirmos algo a respeito da eficiência e robustez dos métodos, preci- 
samos analisá-los usando um conjunto maior e mais representativo de problemas. Pretendemos, como um próximo passo, testar ambos os métodos com os demais problemas da MINLPLib e, dessa forma, inferir algo sobre a robustez e eficiência dos métodos a partir de uma base mais confiável de informação.

Tratamos, também, de um problema de empacotamento de retângulos em regiões convexas arbitrárias. Neste problema, o objetivo é colocar o maior número de retângulos dentro de uma região convexa sem que os retângulos se sobreponham. Consideramos a restrição de que os retângulos deveriam ser posicionados ortogonalmente entre si. Em outras palavras, um lado de cada retângulo deveria ser paralelo a um lado de cada um dos demais retângulos. Por outro lado, permitimos que os retângulos fossem rotacionados em conjunto em relação aos eixos cartesianos. Propusemos, então, um modelo de programação não-linear inteira mista para este problema em que variáveis inteiras têm o papel de determinar se cada um dos retângulos será colocado "em pé" ou "deitado". Realizamos alguns experimentos e resolvemos este problema com o método baseado em Lagrangianos Aumentados e variáveis inteiras que implementamos. Os resultados foram considerados bons e o método foi capaz inclusive de encontrar uma solução de melhor qualidade para uma das instâncias, em comparação com outros métodos conhecidos na literatura. 


\section{Apêndice A}

\section{Convexidade}

A função Lagrangiano Aumentado considerada pelo método Algencan [7, 8, 13, 16] descrito na Seção 3.1 é

$$
L_{\rho}(x, \lambda, \mu)=f(x)+\frac{\rho}{2}\left\{\sum_{i=1}^{m}\left[h_{i}(x)+\frac{\lambda_{i}}{\rho}\right]^{2}+\sum_{i=1}^{p}\left[\max \left(0, g_{i}(x)+\frac{\mu_{i}}{\rho}\right)\right]^{2}\right\}
$$

onde $f: \mathbb{R}^{n} \rightarrow \mathbb{R}, h: \mathbb{R}^{n} \rightarrow \mathbb{R}^{m}$ e $g: \mathbb{R}^{n} \rightarrow \mathbb{R}^{p}$ são funções contínuas e diferenciáveis e $\rho>0, \lambda \in \mathbb{R}^{m}$ e $\mu \in \mathbb{R}_{+}^{p}$. Mostraremos que, para valores fixos de $\rho, \lambda$ e $\mu$, uma condição suficiente para a convexidade de $L_{\rho}(x, \lambda, \mu)$ é que $f$ e $g_{i}$, para $i=1, \ldots, p$, sejam funções convexas e que $h_{i}$, para $i=1, \ldots, m$, sejam funções afins.

\section{A.1 Definição}

Uma função $f: D \rightarrow \mathbb{R}$ é dita convexa se $D$ é um conjunto convexo e

$$
f(\alpha x+(1-\alpha) y) \leq \alpha f(x)+(1-\alpha) f(y)
$$

para todo $x, y \in D$ e para todo $\alpha \in[0,1]$. O Teorema a seguir apresenta a condição necessária e suficiente para a convexidade de funções diferenciáveis.

Teorema A.1 Seja $f: D \rightarrow \mathbb{R}$ uma função diferenciável, onde $D \subseteq \mathbb{R}^{n}$. Então $f$ é uma função convexa se, e somente se, $D$ é um conjunto convexo $e$

$$
f(y) \geq f(x)+\nabla f(x)^{T}(y-x)
$$

para todo $x, y \in D$.

A condição expressa pelo Teorema A.1 é conhecida como condição de convexidade de primeira ordem. 


\section{A.2 Convexidade da função Lagrangiano Aumentado}

Proposiçao A.1 Sejam $f_{1}, f_{2}: D \subset \mathbb{R}^{n} \rightarrow \mathbb{R}$ funções convexas. Então, a função $f$ definida por $f(x)=\max \left(f_{1}(x), f_{2}(x)\right)$ é convexa.

Prova: Sejam $x, y \in \mathbb{R}^{n}$ e $\alpha \in[0,1]$. Então,

$$
\begin{aligned}
f(\alpha x+(1-\alpha) y) & =\max \left(f_{1}(\alpha x+(1-\alpha) y), f_{2}(\alpha x+(1-\alpha) y)\right) \\
& \leq \max \left(\alpha f_{1}(x)+(1-\alpha) f_{1}(y), \alpha f_{2}(x)+(1-\alpha) f_{2}(y)\right) \\
& \leq \max \left(\alpha f_{1}(x), \alpha f_{2}(x)\right)+\max \left((1-\alpha) f_{1}(y),(1-\alpha) f_{2}(y)\right) \\
& =\alpha \max \left(f_{1}(x), f_{2}(x)\right)+(1-\alpha) \max \left(f_{1}(y), f_{2}(y)\right) \\
& =\alpha f(x)+(1-\alpha) f(y) .
\end{aligned}
$$

Logo, $f$ é uma função convexa.

Proposiçao A.2 Seja $f: D \rightarrow \mathbb{R}$ uma função convexa e não negativa, onde $D \subset \mathbb{R}^{n}$. Então, $g(x)=f(x)^{2}$ é uma função convexa.

Prova: Sejam $x, y \in D$ e $\alpha \in[0,1]$. Mostraremos que

$$
f(\alpha x+(1-\alpha) y)^{2} \leq \alpha f(x)^{2}+(1-\alpha) f(y)^{2} .
$$

Como $f$ é convexa, temos

$$
f(\alpha x+(1-\alpha) y) \leq \alpha f(x)+(1-\alpha) f(y) .
$$

Como $f$ é não negativa, temos

$$
\begin{aligned}
f(\alpha x+(1-\alpha) y)^{2} & \leq[\alpha f(x)+(1-\alpha) f(y)]^{2} \\
& =\alpha^{2} f(x)^{2}+2 \alpha(1-\alpha) f(x) f(y)+(1-\alpha)^{2} f(y)^{2} \\
& =\alpha^{2} f(x)^{2}+2 \alpha(1-\alpha) f(x) f(y)+\left[f(y)^{2}-2 \alpha f(y)^{2}+\alpha^{2} f(y)^{2}\right] \\
& =\alpha^{2} f(x)^{2}+2 \alpha(1-\alpha) f(x) f(y)+\left[(1-\alpha) f(y)^{2}-\alpha f(y)^{2}+\alpha^{2} f(y)^{2}\right] .
\end{aligned}
$$

Usando a igualdade $\alpha^{2} f(x)^{2}=\alpha f(x)^{2}-\alpha f(x)^{2}+\alpha^{2} f(x)^{2}$, temos

$$
\begin{aligned}
f(\alpha x+(1-\alpha) y)^{2} \leq & {\left[\alpha f(x)^{2}+(1-\alpha) f(y)^{2}\right]-\alpha f(x)^{2}+\alpha^{2} f(x)^{2} } \\
& +2 \alpha(1-\alpha) f(x) f(y)-\alpha f(y)^{2}+\alpha^{2} f(y)^{2}
\end{aligned}
$$

Para completarmos a prova, basta mostrarmos que

$$
-\alpha f(x)^{2}+\alpha^{2} f(x)^{2}+2 \alpha(1-\alpha) f(x) f(y)-\alpha f(y)^{2}+\alpha^{2} f(y)^{2} \leq 0 .
$$


Temos,

$$
\begin{aligned}
-\alpha f(x)^{2}+\alpha^{2} f(x)^{2}+2 \alpha(1-\alpha) f(x) f(y)-\alpha f(y)^{2}+\alpha^{2} f(y)^{2} & = \\
-\alpha f(x)^{2}+\alpha^{2} f(x)^{2}+2 \alpha f(x) f(y)-2 \alpha^{2} f(x) f(y)-\alpha f(y)^{2}+\alpha^{2} f(y)^{2} & = \\
\alpha^{2}\left[f(x)^{2}-2 f(x) f(y)+f(y)^{2}\right]-\alpha\left[f(x)^{2}-2 f(x) f(y)+f(y)^{2}\right] & = \\
\alpha^{2}[f(x)-f(y)]^{2}-\alpha[f(x)-f(y)]^{2} & = \\
\left(\alpha^{2}-\alpha\right)[f(x)-f(y)]^{2} & \leq 0 .
\end{aligned}
$$

Proposiçao A.3 Seja $f: D \rightarrow \mathbb{R}$ uma função afim, onde $D \subset \mathbb{R}^{n}$ é um conjunto convexo. Então, $g(x)=f(x)^{2}$ é uma função convexa.

Prova: Seja $f(x)=a^{T} x+b$, onde $a \in \mathbb{R}^{n}$ e $b \in \mathbb{R}$. Então, $f(x)^{2}=\left(a^{T} x+b\right)^{2}$. Sejam $x, y \in \mathbb{R}^{n}$. Mostraremos que

$$
f(y)^{2} \geq f(x)^{2}+\nabla f(x)^{2 T}(y-x)
$$

o que, de acordo com o Teorema A.1, indica que $f^{2}$ é uma função convexa. Temos,

$$
\begin{aligned}
f(x)^{2}+\nabla f(x)^{2 T}(y-x) & =\left(a^{T} x+b\right)^{2}+2\left(a^{T} x+b\right) a^{T}(y-x) \\
& =\left(a^{T} x\right)^{2}+2 b a^{T} x+b^{2}+2\left(a^{T} x\right)\left(a^{T} y\right)-2\left(a^{T} x\right)^{2}+2 b a^{T} y-2 b a^{T} x \\
& =b^{2}-\left(a^{T} x\right)^{2}+2\left(a^{T} x\right)\left(a^{T} y\right)+2 b a^{T} y \\
& =b^{2}-\left(a^{T} x\right)^{2}+2\left(a^{T} x\right)\left(a^{T} y\right)+2 b a^{T} y+\left(a^{T} y\right)^{2}-\left(a^{T} y\right)^{2} \\
& =\left(a^{T} y\right)^{2}-\left(a^{T} y-a^{T} x\right)^{2}+2 b a^{T} y+b^{2} \\
& \leq\left(a^{T} y\right)^{2}+2 b a^{T} y+b^{2}=\left(a^{T} y+b\right)^{2}=f(y)^{2} .
\end{aligned}
$$

Logo, $f(y)^{2} \geq f(x)^{2}+\nabla f(x)^{2 T}(y-x)$ e, portanto, $g(x)=f(x)^{2}$ é uma função convexa.

Proposiçao A.4 Sejam $f: \mathbb{R}^{n} \rightarrow \mathbb{R}, g_{i}: \mathbb{R}^{q} \rightarrow \mathbb{R}^{p}$ para $i=1, \ldots, p$ funções convexas e $h_{j}: \mathbb{R}^{q} \rightarrow \mathbb{R}^{m}$ para $j=1, \ldots, m$ funções afins. Então, para valores fixos de $\lambda \in \mathbb{R}^{m}$, $\mu \in \mathbb{R}^{p}$ e $\rho \in \mathbb{R}_{+}^{*}$,

$$
L_{\rho}(x, \lambda, \mu)=f(x)+\frac{\rho}{2}\left\{\sum_{i=1}^{m}\left[h_{i}(x)+\frac{\lambda_{i}}{\rho}\right]^{2}+\sum_{i=1}^{p}\left[\max \left(0, g_{i}(x)+\frac{\mu_{i}}{\rho}\right)\right]^{2}\right\}
$$

é uma função convexa.

Prova: Para $i=1, \ldots, p$, como $g_{i}$ é uma função convexa, $g_{i}(x)+\frac{\mu_{i}}{\rho}$ também é uma função convexa. Portanto, pela Proposição A.1, $m_{i}(x)=\max \left(0, g_{i}(x)+\frac{\mu_{i}}{\rho}\right)$ é uma função 
convexa, para $i=1, \ldots, p$. Como $m_{i}(x)$ é convexa e não negativa, então $m_{i}^{2}(x)$ é uma função convexa pela Proposição A.2. Logo,

$$
\sum_{i=1}^{p}\left[\max \left(0, g_{i}(x)+\frac{\mu_{i}}{\rho}\right)\right]^{2}
$$

é uma função convexa (já que uma combinação positiva de funções convexas é uma função convexa). Como $h_{i}(x)+\frac{\lambda_{i}}{\rho}$ é uma função afim, para $i=1, \ldots, m$, então, pela Proposição A.3, $\left[h_{i}(x)+\frac{\lambda_{i}}{\rho}\right]^{2}$ é uma função convexa, para $i=1, \ldots, m$. Logo,

$$
\sum_{i=1}^{m}\left[h_{i}(x)+\frac{\lambda_{i}}{\rho}\right]^{2}
$$

é uma função convexa. Portanto, sendo $f$ uma função convexa e $\rho>0$,

$$
f(x)+\frac{\rho}{2}\left\{\sum_{i=1}^{m}\left[h_{i}(x)+\frac{\lambda_{i}}{\rho}\right]^{2}+\sum_{i=1}^{p}\left[\max \left(0, g_{i}(x)+\frac{\mu_{i}}{\rho}\right)\right]^{2}\right\}
$$

é uma função convexa. 


\section{Apêndice B}

\section{Problemas}

Alguns problemas encontrados na literatura e usados nos testes mostrados no Capítulo 3.3. Os problemas numerados de 1 a 6 também estão reunidos em [33].

Problema 1. Exemplo 3 de Grossmann e Kocis [44]. Exemplo 12.2.1 de Floudas et al. [33].

$$
\begin{array}{lrl}
\text { Minimizar } & 2 x_{1}+3 x_{2}+1.5 y_{1}+2 y_{2}-0.5 y_{3} \\
\text { sujeita a } & x_{1}^{2}+y_{1} & =1.25 \\
x_{2}^{1.5}+1.5 y_{2} & =3 \\
x_{1}+y_{1} & \leq 1.6 \\
1.333 x_{2}+y_{2} & \leq 3 \\
-y_{1}-y_{2}+y_{3} & \leq 0 \\
x_{1}, x_{2} & \geq 0 \\
y & \in\{0,1\}^{3}
\end{array}
$$

Solução global: $\quad x^{*}=(1.12,1.31)^{T}$

Valor ótimo: $\quad 7.6672$

$$
y^{*}=(0,1,1)^{T}
$$

Problema 2. Exemplo 6.6.5 de Floudas [31] e 12.2.2 de Floudas et al. [33].

$$
\begin{array}{lrl}
\text { Minimizar } & -0.7 y+5\left(x_{1}-0.5\right)^{2}+0.8 \\
\text { sujeita a } & -e^{\left(x_{1}-0.2\right)}-x_{2} \leq 0 \\
& x_{2}+1.1 y \leq-1 \\
x_{1}-1.2 y & \leq 0.2 \\
0.2 \leq & x_{1} \leq 1 \\
& -2.22554 \leq x_{2} \leq-1 \\
y & \in\{0,1\}
\end{array}
$$

Solução global: $\quad x^{*}=(0.9419,-2.1)^{T}$

$y^{*}=1$

Valor ótimo: $\quad 1.0765$ 
Observação: No livro de Floudas et al. [33], a segunda restrição é

$$
x_{2}+1.1 y \leq 1 .
$$

Mas a restrição correta é

$$
x_{2}+1.1 y \leq-1 .
$$

Problema 3. Problema extraído de Yuan et al. [69]. Exemplo 12.2.3 de Floudas et al. [33].

$$
\begin{aligned}
& \text { Minimizar } \quad\left(y_{1}-1\right)^{2}+\left(y_{2}-2\right)^{2}+\left(y_{3}-1\right)^{2}-\ln \left(y_{4}+1\right)+ \\
& \text { sujeita a } \\
&\left(x_{1}-1\right)^{2}+\left(x_{2}-2\right)^{2}+\left(x_{3}-3\right)^{2} \\
& 2 y_{1}+y_{2}+y_{3}+x_{1}+x_{2}+x_{3} \leq 5 \\
& y_{3}^{2}+x_{1}^{2}+x_{2}^{2}+x_{3}^{2} \leq 5.5 \\
& y_{1}+x_{1} \leq 1.2 \\
& y_{2}+x_{2} \leq 1.8 \\
& y_{3}+x_{3} \leq 2.5 \\
& y_{4}+x_{1} \leq 1.2 \\
& y_{2}^{2}+x_{2}^{2} \leq 1.64 \\
& y_{3}^{2}+x_{3}^{2} \leq 4.25 \\
& y_{2}^{2}+x_{3}^{2} \leq 4.64 \\
& x_{1}, x_{2}, x_{3} \geq 0 \\
& y \in\{0,1\}^{4}
\end{aligned}
$$

Ponto inicial: $\quad y=(0,1,1,0)^{T}$

Solução global: $\quad x^{*}=(0.2,0.8,1.908)^{T}$

Valor ótimo: $\quad 5.5796$$$
y^{*}=(0,1,0,1)^{T}
$$

Observação: No livro de Floudas et al. [33], a primeira e a segunda restrições são

$$
\begin{aligned}
y_{1}+y_{2}+y_{3}+x_{1}+x_{2}+x_{3} & \leq 5 \\
y_{3}^{2}+x_{1}^{2}+x_{2}^{2}+x_{3}^{2} & \leq 5.5
\end{aligned}
$$

e a solução ótima reportada é $x^{*}=(0.2,0.8,1.908)^{T}, y^{*}=(1,1,0,1)^{T}$ com valor 4.5796 . Já no artigo de Yuan et al. [69], onde o problema foi originalmente publicado, as duas primeiras restrições são

$$
\begin{aligned}
\mathbf{2} y_{1}+y_{2}+y_{3}+x_{1}+x_{2}+x_{3} & \leq 5 \\
y_{3}^{2}+x_{1}^{2}+x_{2}^{2}+x_{3}^{\mathbf{3}} & \leq 5.5
\end{aligned}
$$

e a solução global reportada é $x^{*}=(0.2,0.8,1.908)^{T}, y^{*}=(0,1,0,1)^{T}$ com valor 5.5796 . Porém, essa solução é inviável, já que ela não satisfaz a segunda restrição. O que fizemos foi considerar as duas seguintes restrições

$$
\begin{aligned}
\mathbf{2} y_{1}+y_{2}+y_{3}+x_{1}+x_{2}+x_{3} & \leq 5 \\
y_{3}^{2}+x_{1}^{2}+x_{2}^{2}+x_{3}^{2} & \leq 5.5
\end{aligned}
$$


que tornam a solução publicada por Yuan et al. [69] viável.

Problema 4. Exemplo 12.2.4 de Floudas et al. [33].

$$
\begin{aligned}
& \text { Minimizar }-x_{1} x_{2} x_{3} \\
& \text { sujeita a } \quad x_{1}+0.1^{y_{1}} 0.2^{y_{2}} 0.15^{y_{3}}=1 \\
& x_{2}+0.05^{y_{4}} 0.2^{y_{5}} 0.15^{y_{6}}=1 \\
& x_{3}+0.02^{y_{7}} 0.06^{y_{8}}=1 \\
& -y_{1}-y_{2}-y_{3} \leq-1 \\
& -y_{4}-y_{5}-y_{6} \leq-1 \\
& -y_{7}-y_{8} \leq-1 \\
& 3 y_{1}+y_{2}+2 y_{3}+3 y_{4}+2 y_{5}+y_{6}+3 y_{7}+2 y_{8} \leq 10 \\
& x_{1}, x_{2}, x_{3} \leq 1 \\
& x_{1}, x_{2}, x_{3} \geq 0 \\
& y \in\{0,1\}^{8}
\end{aligned}
$$

Solução global: $\quad x^{*}=(0.97,0.9925,0.98)^{T}$

Valor ótimo: $\quad-0.94347$

$$
y^{*}=(0,1,1,1,0,1,1,0)^{T}
$$

Problema 5. Proposto por Harjunkoski et al. [53]. Exemplo 12.2.5 de Floudas et al. [33].

$$
\begin{array}{lr}
\text { Minimizar } & 7 y_{1}+10 y_{2} \\
\text { sujeita a } & y_{1}^{1.2} y_{2}^{1.7}-7 y_{1}-9 y_{2}+24 \\
-y_{1}-2 y_{2}+5 & \leq 0 \\
-3 y_{1}+y_{2}-1 & \leq 0 \\
4 y_{1}-3 y_{2}-11 & \leq 0 \\
y_{1}, y_{2} & \in\{1,2,3,4,5\}
\end{array}
$$

Solução global: $\quad y^{*}=(3,1)^{T}$

Valor ótimo: $\quad 31$

Observação: No livro de Floudas et al. [33], as duas primeiras restrições são

$$
\begin{aligned}
y_{1}^{1.2} y_{2}^{1.7}-7 y_{1}-9 y_{2} & \leq 24 \\
-y_{1}-2 y_{2} & \leq 5
\end{aligned}
$$

o que não está de acordo com o artigo de Harjunkoski et al. [53], em que essas restrições são

$$
\begin{aligned}
y_{1}^{1.2} y_{2}^{1.7}-7 y_{1}-9 y_{2}+24 & \leq 0 \\
-y_{1}-2 y_{2}+5 & \leq 0
\end{aligned}
$$

Em nossos experimentos consideramos essas duas últimas restrições. 
Problema 6. Proposto por Harjunkoski et al. [53]. Exemplo 12.2.6 de Floudas et al. [33].

$$
\begin{aligned}
& \text { Minimizar } 3 y-5 x \\
& \text { sujeita a } 2 y^{2}-2 y^{0.5}-2 x^{0.5} y^{2}+11 y+8 x-39 \leq 0 \\
& -y+x-3 \leq 0 \\
& 2 y+3 x-24 \leq 0 \\
& 1 \leq x \leq 10 \\
& y \in\{1,2,3,4,5,6\}
\end{aligned}
$$

Ponto inicial: $\quad x=1$

$$
y=1
$$

Solução global: $x^{*}=4$

$y^{*}=1$

Valor ótimo: $\quad-17$

Observação: No artigo de Harjunkoski et al. [53], há um erro na apresentação do modelo. A segunda restrição está

$$
-x+x-3 \leq 0
$$

mas o correto é

$$
-y+x-3 \leq 0 .
$$

Problema 7. Síntese de rede de trocadores de calor. Exemplo 12.3.3 de Floudas et al. [33].

Problema 8. Síntese de rede de trocadores de calor. Exemplo 4 de Zamora e Grossmann [50] e exemplo 12.4.3 de Floudas et al. [33].

Problema 9. Síntese de rede de trocadores de calor. Exemplo 5 de Zamora e Grossmann [50] e exemplo 12.4.4 de Floudas et al. [33].

Problema 10. Exemplo 12.5.2 de Floudas et al. [33] e exemplo de Grossmann et al. [45]. 
Problema 11. Minimização de desperdício em cortes de papel. Floudas et al. [33]. Abaixo temos a formulação geral do problema e, em seguida, os dados para quatro problemas específicos.

$$
\begin{aligned}
& \text { Minimizar } \quad \sum_{j=1}^{P} m_{j}+0.1 j y_{j} \\
& \text { sujeita a } \\
& \sum_{j=1}^{P} m_{j} r_{i j} \geq n_{i}, \quad i=1, \ldots, N \\
& \left(B_{\max }-\Delta\right) y_{j}-\sum_{i=1}^{N} b_{i} r_{i j} \leq 0, \quad j=1, \ldots, P \\
& \sum_{i=1}^{N} b_{i} r_{i j}-B_{\text {max }} y_{j} \leq 0, \quad j=1, \ldots, P \\
& y_{j}-\sum_{i=1}^{N} r_{i j} \leq 0, \quad j=1, \ldots, P \\
& \sum_{i=1}^{N} r_{i j}-N k_{\max } y_{j} \leq 0, \quad j=1, \ldots, P \\
& y_{j}-m_{j} \leq 0, \quad j=1, \ldots, P \\
& m_{j}-M_{j} y_{j} \leq 0, \quad j=1, \ldots, P \\
& \sum_{j=1}^{P} m_{j} \geq \max \left\{\left\lceil\frac{\sum_{i=1}^{N} n_{i}}{N k_{\max }}\right\rceil,\left\lceil\frac{\sum_{i=1}^{N} b_{i} n_{i}}{B_{\max }}\right\rceil\right\} \\
& y_{k+1}-y_{k} \leq 0, \quad k=1, \ldots, P-1 \\
& m_{k+1}-m_{k} \leq 0, \quad k=1, \ldots, P-1 \\
& y \in\{0,1\}^{P} \\
& m_{j} \in\left[0, M_{j}\right] \cap \mathbb{Z}, j=1, \ldots, P \\
& r_{i j} \in\left[0, N k_{\text {max }}\right] \cap \mathbb{Z}, i=1, \ldots, N \text {, } \\
& j=1, \ldots, P
\end{aligned}
$$

\section{Problema 11.1}

Dados: $\quad N=4, P=4, B_{\max }=1850 \mathrm{~mm}, \Delta=100 \mathrm{~mm}$

$$
\begin{aligned}
& n=(15,28,21,30)^{T} \\
& b=(290,315,350,455)^{T} \\
& M=(30,30,30,30)^{T}
\end{aligned}
$$

Solução global: $y^{*}=(1,1,1,0)^{T}$

$$
\begin{aligned}
m^{*}= & (9,7,3,0)^{T} \\
r= & \left(\begin{array}{llll}
1 & 0 & 2 & 0 \\
2 & 1 & 1 & 0 \\
0 & 3 & 0 & 0 \\
2 & 1 & 2 & 0
\end{array}\right)
\end{aligned}
$$

Valor ótimo: 19.6 


\section{Problema 11.2}

Dados: $\quad N=4, P=4, B_{\max }=1900 \mathrm{~mm}, \Delta=200 \mathrm{~mm}$

$$
\begin{aligned}
& n=(9,7,12,11)^{T} \\
& b=(330,360,385,415)^{T} \\
& M=(15,12,9,6)^{T}
\end{aligned}
$$

Solução global: $y^{*}=(1,0,0,0)^{T}$

$$
\begin{aligned}
m^{*}= & (11,0,0,0)^{T} \\
r= & \left(\begin{array}{llll}
1 & 0 & 0 & 0 \\
1 & 0 & 0 & 0 \\
2 & 0 & 0 & 0 \\
1 & 0 & 0 & 0
\end{array}\right)
\end{aligned}
$$

Valor ótimo: 8.6

\section{Problema 11.3}

Dados: $\quad N=5, P=5, B_{\max }=2000 \mathrm{~mm}, \Delta=200 \mathrm{~mm}$

$$
\begin{aligned}
& n=(12,6,15,6,9)^{T} \\
& b=(330,360,370,415,435)^{T} \\
& M=(15,12,9,6,6)^{T}
\end{aligned}
$$

Solução global: $y^{*}=(1,0,0,0,0)^{T}$

$$
\begin{aligned}
m^{*} & =(15,0,0,0,0)^{T} \\
r_{i 1} & =1, i=1, \ldots, 5 \\
r_{i j} & =0, i=1, \ldots, 5, j=2, \ldots, 5
\end{aligned}
$$

Valor ótimo: 10.3

\section{Problema 11.4}

Dados: $\quad N=6, P=6, B_{\max }=2200 \mathrm{~mm}, \Delta=100 \mathrm{~mm}$

$$
\begin{aligned}
& n=(8,16,15,7,14,16)^{T} \\
& b=(330,360,380,430,490,530)^{T} \\
& M=(15,12,8,7,4,2)^{T}
\end{aligned}
$$

Solução global: $y^{*}=(1,1,0,0,0,0)^{T}$

$$
\begin{aligned}
m^{*}= & (8,7,0,0,0,0)^{T} \\
r= & \left(\begin{array}{llllll}
1 & 0 & 0 & 0 & 0 & 0 \\
2 & 0 & 0 & 0 & 0 & 0 \\
0 & 2 & 0 & 0 & 0 & 0 \\
0 & 1 & 0 & 0 & 0 & 0 \\
0 & 2 & 0 & 0 & 0 & 0 \\
1 & 0 & 0 & 0 & 0 & 0
\end{array}\right)
\end{aligned}
$$

Valor ótimo: 8.6 
Problema 12. Síntese de processos. Problema 1 de Duran e Grossmann [26].

$$
\begin{aligned}
& \text { Minimizar } 5 y_{1}+6 y_{2}+8 y_{3}+10 x_{1}-7 x_{3} \\
& -18 \ln \left(x_{2}+1\right)-19.2 \ln \left(x_{1}-x_{2}+1\right)+10 \\
& \text { sujeita a } 0.8 \ln \left(x_{2}+1\right)+0.96 \ln \left(x_{1}-x_{2}+1\right)-0.8 x_{3} \geq 0 \\
& x_{2}-x_{1} \leq 0 \\
& x_{2}-U y_{1} \leq 0 \\
& x_{1}-x_{2}-U y_{2} \leq 0 \\
& \ln \left(x_{2}+1\right)+1.2 \ln \left(x_{1}-x_{2}+1\right)-x_{3}-U y_{3} \geq-2 \\
& y_{1}+y_{2} \leq 1 \\
& \begin{array}{rll}
y & \in & \{0,1\}^{3} \\
(0,0,0)^{T} \leq & x \leq(2,2,1)^{T} \\
U & =2
\end{array}
\end{aligned}
$$

Ponto inicial: $\quad y=(1,0,1)^{T}$

Solução global: $\quad x^{*}=(1.30097,0,1)^{T}$

$y^{*}=(0,1,0)^{T}$

Valor ótimo:

$$
6.00972
$$

Problema 13. Síntese de processos. Problema 2 de Duran e Grossmann [26].

$$
\text { Minimizar } \begin{aligned}
& 5 y_{1}+8 y_{2}+6 y_{3}+10 y_{4}+6 y_{5} \\
&-10 x_{1}-15 x_{2}-15 x_{5}+15 x_{3}+5 x_{4}-20 x_{6} \\
&+e^{x_{1}}+e^{x_{2} / 1.2}-60 \ln \left(x_{3}+x_{4}+1\right)+140 \\
&-\ln \left(x_{3}+x_{4}+1\right) \leq 0 \\
& \text { sujeita a } \quad-x_{1}-x_{2}-2 x_{5}+x_{3}+2 x_{6} \leq 0 \\
&-x_{1}-x_{2}-0.75 x_{5}+x_{3}+2 x_{6} \leq 0 \\
& x_{5}-x_{6} \leq 0 \\
& 2 x_{5}-x_{3}-2 x_{6} \leq 0 \\
&-0.5 x_{3}+x_{4} \leq 0 \\
& 0.2 x_{3}-x_{4} \leq 0 \\
& e^{x_{1}}-U y_{1} \leq 1 \\
& e^{x_{2} / 1.2}-U y_{2} \leq 1 \\
& 1.25 x_{5}-U y_{3} \leq 0 \\
& x_{3}+x_{4}-U y_{4} \leq 0 \\
&-2 x_{5}+2 x_{6}-U y_{5} \leq 0 \\
& y_{1}+y_{2}=1 \\
& y_{4}+y_{5} \leq 1 \\
& U=10 \\
& \leq x \leq(2,2,2,+\infty,+\infty, 3)^{T} \\
& y \in\{0,1\}^{5} \\
& x \in \mathbb{R}^{6}
\end{aligned}
$$

Ponto inicial: $\quad y=(1,0,0,0,0)^{T}$

Solução global: $\quad x^{*}=(0,2,0.65201,0.32601,1.07839,1.07839)^{T}$

$y^{*}=(0,1,1,1,0)^{T}$

Valor ótimo: $\quad 73.0353$ 
Problema 14. Síntese de processos. Problema 3 de Duran e Grossmann [26].

$$
\begin{aligned}
& \text { Minimizar } 5 y_{1}+8 y_{2}+6 y_{3}+10 y_{4}+6 y_{5}+7 y_{6}+4 y_{7}+5 y_{8} \\
& -10 x_{1}-15 x_{2}+15 x_{3}+80 x_{4}+25 x_{5}+35 x_{6}-40 x_{7}+15 x_{8}-35 x_{9} \\
& +e^{x_{1}}+e^{x_{2} / 1.2}-65 \ln \left(x_{3}+x_{4}+1\right)-90 \ln \left(x_{5}+1\right)-80 \ln \left(x_{6}+1\right)+120 \\
& \text { sujeita a } \\
& -1.5 \ln \left(x_{5}+1\right)-\ln \left(x_{6}+1\right)-x_{8} \leq 0 \\
& -\ln \left(x_{3}+x_{4}+1\right) \leq 0 \\
& -x_{1}-x_{2}+x_{3}+2 x_{4}+0.8 x_{5}+0.8 x_{6}-0.5 x_{7}-x_{8}-2 x_{9} \leq 0 \\
& -x_{1}-x_{2}+2 x_{4}+0.8 x_{5}+0.8 x_{6}-2 x_{7}-x_{8}-2 x_{9} \leq 0 \\
& -2 x_{4}-0.8 x_{5}-0.8 x_{6}+2 x_{7}+x_{8}+2 x_{9} \leq 0 \\
& -0.8 x_{5}-0.8 x_{6}+x_{8} \leq 0 \\
& -x_{4}+x_{7}+x_{9} \leq 0 \\
& -0.4 x_{5}-0.4 x_{6}+1.5 x_{8} \leq 0 \\
& 0.16 x_{5}+0.16 x_{6}-1.2 x_{8} \leq 0 \\
& x_{3}-0.8 x_{4} \leq 0 \\
& -x_{3}+0.4 x_{4} \leq 0 \\
& e^{x_{1}}-U y_{1} \leq 1 \\
& e^{x_{2} / 1.2}-U y_{2} \leq 1 \\
& x_{7}-U y_{3} \leq 0 \\
& 0.8 x_{5}+0.8 x_{6}-U y_{4} \leq 0 \\
& 2 x_{4}-2 x_{7}-2 x_{9}-U y_{5} \leq 0 \\
& x_{5}-U y_{6} \leq 0 \\
& x_{6}-U y_{7} \leq 0 \\
& x_{3}+x_{4}-U y_{8} \leq 0 \\
& y_{1}+y_{2}=1 \\
& y_{4}+y_{5} \leq 1 \\
& -y_{4}+y_{6}+y_{7}=0 \\
& y_{3}-y_{8} \leq 0 \\
& U=10 \\
& x \geq 0 \\
& x_{i} \leq 2, i \neq 3,8,9 \\
& x_{3}, x_{8} \leq 1 \\
& x_{9} \leq 3 \\
& y \in\{0,1\}^{8} \\
& x \in \mathbb{R}^{9}
\end{aligned}
$$

Solução global: $\quad x^{*}=(0,2,0.46784,0.58480,2,0,0,0.26667,0.58480)^{T}$

$y^{*}=(1,0,0,1,0,1,0,0)^{T}$

Valor ótimo: $\quad 68.0097$ 


\section{Referências Bibliográficas}

[1] T. Achterberg, T. Koch, and A. Martin. Branching on history information. Technical Report 02-32, Konrad-Zuse-Zentrum für Informationstechnik Berlin, 2002.

[2] T. Achterberg, T. Koch, and A. Martin. Branching rules revisited. Operations Research Letters, 33(1):42-54, 2005.

[3] C. S. Adjiman, I. P. Androulakis, and C. A. Floudas. Global optimization of MINLP problems in process synthesis and design. Computers \& Chemical Engineering, 21:S445-S450, 1997.

[4] C. S. Adjiman, I. P. Androulakis, and C. A. Floudas. Global Optimization of MixedInteger Nonlinear Problems. AIChE Journal, 46(9):1769-1797, 2000.

[5] C. S. Adjiman, C. A. Floudas, and C. A. Schweiger. Handbook of Combinatorial Optimization, volume 1, chapter Mixed-Integer Nonlinear Optimization in Process Synthesis. Kluwer Academic Publisher, 1999.

[6] A. Aggarwal, A. R. Ciric, and C. A. Floudas. Global optimal search for nonconvex NLP and MINLP problems. Computers \&3 Chemical Engineering, 13:1117, 1989.

[7] R. Andreani, E. G. Birgin, J. M. Martínez, and M. L. Schuverdt. On Augmented Lagrangian methods with general lower-level constraints. SIAM Journal on Optimization, 18:1286-1309, 2007.

[8] R. Andreani, E. G. Birgin, J. M. Martínez, and M. L. Schuverdt. Augmented Lagrangian methods under the Constant Positive Linear Dependence constraint qualification. Mathematical Programming, 111:5-32, 2008.

[9] M. Andretta, E. G. Birgin, and J. M. Martínez. Practical active-set Euclidian trustregion method with spectral projected gradients for bound-constrained minimization. Optimization, 54:305-325, 2005.

[10] E. M. L. Beale. Integer Programming. The State of the Art in Numerical Analysis, pages 409-448, 1977.

[11] E. M. L. Beale and J. A. Tomlin. Special facilities in a general mathematical programming system for nonconvex problems using ordered sets of variables. In J. Lawrence, 
editor, Fifth International Conference on Operations Research, pages 447-454, London, 1970. Tavistock Publications.

[12] M. Benichou, J. -M. Gauthier, P. Girodet, G. Hentges, G. Ribière, and D. Vincent. Experiments in mixed-integer linear programming. Mathematical Programming, 1:7694, 1971.

[13] E. G. Birgin, R. Castillo, and J. M. Martínez. Numerical comparison of augmented lagrangian algorithms for nonconvex problems. Computational Optimization and Applications, 31:31-56, 2005.

[14] E. G. Birgin, C. A. Floudas, and J. M. Martínez. Global minimization using an Augmented Lagrangian method with variable lower-level constraints. Mathematical Programming, 2009, to appear. Disponível em Optimization on Line e em http://www.ime.usp.br/ egbirgin/.

[15] E. G. Birgin and J. M. Martínez. Large-scale active-set box-constrained optimization method with spectral projected gradients. Computational Optimization and Applications, 23(1):101-125, 2002.

[16] E. G. Birgin and J. M. Martínez. Structured minimal-memory inexact quasi-Newton method and secant preconditioners for Augmented Lagrangian Optimization. Computational Optimization and Applications, 39:1-16, 2008.

[17] E. G. Birgin, J. M. Martínez, W. F. Mascarenhas, and D. P. Ronconi. Method of sentinels for packing items within arbitrary convex regions. Journal of the Operational Research Society, 57(6):735-746, 2006.

[18] E. G. Birgin, J. M. Martínez, F. H. Nishihara, and D. P. Ronconi. Orthogonal packing of rectangular items within arbitrary convex regions by nonlinear optimization. Computers \& Operations Research, 33(12):3535-3548, 2006.

[19] P. Bonami, L. T. Biegler, A. R. Conn, G. Cornuéjols, I. E. Grossmann, C. D. Laird, J. Lee, A. Lodi, F. Margot, N. Sawaya, and A. Wächter. An algorithmic framework for convex mixed integer nonlinear programs. Discrete Optimization, 5(2):186-204, 2008.

[20] B. Borchers and J. E. Mitchell. An improved branch and bound algorithm for mixed integer nonlinear programs. Comput. Oper. Res., 21(4):359-367, 1994.

[21] A. Brooke, D. Kendrick, and A. Meeraus. GAMS: A User's Guide. The Scientific Press, 1992.

[22] Michael R. Bussieck, Arne Stolbjerg Drud, and Alexander Meeraus. MINLPLibA Collection of Test Models for Mixed-Integer Nonlinear Programming. INFORMS Journal on Computing, 15(1):114-119, 2003.

[23] COIN-OR. http://www.coin-or.org/. 
[24] CONVERT. http://www.gamsworld.org/performance/paver/convert_submit.htm.

[25] E. Dolan and J. J. Moré. Benchmarking optimization software with performance profiles. Mathematical Programming, 91(2):201-213, 2002.

[26] M. A. Duran and I. E. Grossmann. An outer-approximation algorithm for a class of mixed-integer nonlinear programs. Mathematical Programming, 36:307-339, 1986.

[27] J. Eckstein. Parallel Branch-and-Bound Algorithms for General mixed Integer Programming on the CM-5. SIAM Journal on Optimization, 4(4):794-814, 1994.

[28] R. Fletcher. Practical methods of optimization. Wiley-Interscience, New York, NY, USA, second edition, 1987.

[29] R. Fletcher and S. Leyffer. Solving mixed integer nonlinear programs by outer approximation. Mathematical Programming, 66:327, 1994.

[30] R. Fletcher and S. Leyffer. Numerical experience with lower bounds for MIQP Branchand-Bound. SIAM J. Optim., 8(2):604-616, 1998.

[31] C. A. Floudas. Nonlinear and Mixed-Integer Optimization: Fundamentals and Applications. Oxford University Press, New York, 1995.

[32] C. A. Floudas and X. Lin. Design, synthesis and scheduling of multipurpose batch plants via an effective continuous-time formulation. Computers \& Chemical Engineering, 25:665-674, 2001.

[33] C. A. Floudas, P. M. Pardalos, C. Adjiman, W. R. Esposito, Z. H. Gümüs, S. T. Harding, J. L. Klepeis, C. A. Meyer, and C. A. Schweiger. Handbook of Test Problems in Local and Global Optimization. Nonconvex Optimization and its Applications. Kluwer Academic Publishers, Dordrecht, The Netherlands, 1999.

[34] R. Fourer, D. M. Gay, and B. W. Kernighan. AMPL - A Modeling Language for Mathematical Programming. Duxbury Press, 2nd edition, 2002.

[35] R. S. Garfinkel and G. L. Nemhauser. Integer Programming. John Wiley \& Sons, New York, 1972. Series in Decision and Control.

[36] J. -M. Gauthier and G. Ribière. Experiments in mixed-integer linear programming using pseudo-costs. Mathematical Programming, 12:26-47, 1977.

[37] B. Gavish, D. Horsky, and K. Srikanth. An Approach to the Optimal Positioning of a New Product. Management Science, 29(11):1277-1297, 1983.

[38] David M. Gay. Hooking your solver to AMPL. Numerical Analysis Manuscript No. 93-10, AT\&T Bell Laboratories, Murray Hill, NJ, 1993, revised 1997.

[39] A. M. Geoffrion. Generalized Benders decomposition. Journal of Optimization Theory and Applications, 10:237-260, 1972. 
[40] N. F. Giannelos and M. C. Georgiadis. A Model for Scheduling Cutting Operations in Paper-Converting Processes. Industrial \& Engineering Chemistry Research, 40(24):5752-5757, 2001.

[41] W. Glankwamdee and J. T. Linderoth. Lookahead Branching for Mixed Integer Programming. Technical Report 06T-004, Industrial and Systems Engineering, Lehigh University, 2006.

[42] J.-P. Goux and S. Leyffer. Solving large MINLPs on computational grids. Optimization and Engineering, 3(3):327-346, 2002.

[43] I. E. Grossmann and G. R. Kocis. Relaxation estrategy for the structural optimization of process flow sheets. Ind. Eng. Chem., 26, 1987.

[44] I. E. Grossmann and G. R. Kocis. Global Optimization of Nonconvex Mixed-Integer Nonlinear Programming (MINLP) Problems in Process Synthesis. Industrial \&6 Engineering Chemistry Research, 27:1407-1421, 1988.

[45] I. E. Grossmann, F. Pettersson, and T. Westerlund. Optimization of pump configurations as a MINLP problem. Computers \&5 Chemical Engineering, 18(9):845-858, 1994.

[46] I. E. Grossmann and I. Quesada. An LP/NLP based branch and bound algorithm for convex MINLP optimization problems. Computers \& Chemical Engineering, 16:937947, 1992.

[47] I. E. Grossmann and R. W. H. Sargent. Optimal design of multipurpose batch plants. Ind. Engng. Chem. Process Des. Dev., 18:343-348, 1979.

[48] I. E. Grossmann and J. Viswanathan. A combined penalty function and outer approximation method for MINLP optimization. Computers \& Chemical Engineering, 14:769-782, 1990.

[49] I. E. Grossmann and J. Viswanathan. Optimal feed location and number of trays for distillation columns with multiple feeds. IEEEC Research, 32:2942-2949, 1993.

[50] I. E. Grossmann and J. M. Zamora. A global MINLP optimization algorithm for the synthesis of heat exchanger networks with no stream splits. Computers $\mathscr{E}$ Chemical Engineering, 22(3):367-384, 1998.

[51] O. K. Gupta and A. Ravindran. Branch and bound experiments in convex nonlinear integer programming. Management Science, 31:1533-1546, 1985.

[52] I. Harjunkoski, R. Pörn, H. Skrifvars, and T. Westerlund. Different transformations for solving non-convex trim-loss problems by minlp. European Journal of Operational Research, 105:594-603, 16 March 1998.

[53] I. Harjunkoski, R. Pörn, and T. Westerlund. Convexification of different classes of non-convex MINLP problems. Computers \& Chemical Engineering, 23:439-448, 1999. 
[54] M. R. Hestenes. Multiplier and gradient methods. Journal of Optimization Theory and Applications, 4(5):303-320, 1969.

[55] J. Isaksson, I. Harjunkoski, and T. Westerlund. Solving a production optimization problem in a paper-converting mill with MILP. Computers $\&$ Chemical Engineering, 22(4):563-570, 1998.

[56] V. D. Kosmidis, J. D. Perkins, and E. N. Pistikopoulos. A mixed integer optimization formulation for the well scheduling problem on petroleum fields. Computers 85 Chemical Engineering, 29(7):1523-1541, 2005.

[57] A. H. Land and A. G. Doig. An Automatic Method of Solving Discrete Programming Problems. Econometrica, 28(3):497-520, 1960.

[58] D. J. Laughhunn. Quadratic binary programming with applications to capital budgeting problems. Operations Research, 18(3):454-461, 1970.

[59] J. T. Linderoth and M. W. P. Savelsbergh. A Computational Study of Search Strategies for Mixed Integer Programming. INFORMS Journal on Computing, 11(2):173$187,1999$.

[60] G. Mitra. Investigation of some branch and bound strategies for the solution of mixed integer linear programs. Mathematical Programming, 4(1), 1973.

[61] G. L. Nemhauser and L. A. Wolsey. Integer and Combinatorial Optimization. John Wiley \& Sons, 1988.

[62] O. Odele and S. Macchietto. Computer aided molecular design: a novel method for optimal solvent selection. Fluid Phase Equilibria, 82:39-46, 1993.

[63] G. M. Ostrovsky, M. G. Ostrovsky, and G. W. Mikhailow. Discrete optimization of chemical processes. Computers \& Chemical Engineering, 14:111-117, 1990.

[64] M. J. D. Powell. A method for nonlinear constraints in minimization problems. In R. Fletcher, editor, Optimization, pages 283-298. Academic Press, New York, NY, 1969 .

[65] R. T. Rockafellar. The multiplier method of Hestenes and Powell applied to convex programming. Journal of Optimization Theory and Applications, 12(6):555-562, 1973.

[66] N. V. Sahinidis and M. Tawarmalani. Applications of global optimization to process and molecular design. Computers \& Chemical Engineering, 24(9):2157-2169, 2000.

[67] L. Schrage. A More Portable Fortran Random Number Generator. ACM Transactions on Mathematical Software, 5(2):132-138, 1979.

[68] M. Tawarmalani and N. Sahinidis. Convexification and Global Optimization in Continuous and Mixed-Integer Nonlinear Programming: Theory, Algorithms, Software, and Application. Kluwer Academic Publishers, Dordrecht, The Netherlands, 2002. 
[69] X. Yuan, S. Zhang, L. Pibouleau, and S. Domenech. Une methode d'optimisation non lineaire en variables mixtes pour la conception de procedes. Recherche Operationnelle/Operations Research, 22(4):331-346, 1988. 